\title{
CONTINUOUS SYMMETRIZATION VIA POLARIZATION
}

\author{
ALEXANDER YU. SOLYNIN
}

\begin{abstract}
We discuss a one-parameter family of transformations which changes sets and functions continuously into their $(k, n)$-Steiner symmetrizations. Our construction consists of two stages. First, we employ a continuous symmetrization introduced by the author in 1990 to transform sets and functions into their onedimensional Steiner symmetrization. Some of our proofs in this stage rely on a simple rearrangement called polarization.

In the second stage, we use an approximation theorem due to Blaschke and Sarvas to give an inductive definition of the continuous $(k, n)$-Steiner symmetrization for any $2 \leq k \leq n$. This transformation provides us with the desired continuous path, along which all basic characteristics of sets and functions vary monotonically. The latter leads to continuous versions of several convolution type inequalities and Dirichlet's type inequalities as well as to continuous versions of comparison theorems for solutions of some elliptic and parabolic partial differential equations.
\end{abstract}

\section{INTRODUCTION}

The first geometric transformation bearing the name symmetrization was introduced by Jacob Steiner in 1836 [30] in one of his attempts to find a rigorous proof for the classical isoperimetric problem. Let $C$ be a closed contour on $\mathbb{R}^{2}$ enclosing a domain $D$ and let $m_{D}(x)$ denote the Lebesgue measure of the intersection of $D$ with the vertical line $v_{x}=\left\{(x, y) \in \mathbb{R}^{2}:-\infty<y<\infty\right\}$. Then Steiner's symmetrization of $D$ with respect to the $x$-axis is defined by

$$
D^{*}=\left\{(x, y) \in \mathbb{R}^{2}:|y|<(1 / 2) m_{D}(x)\right\} .
$$

This implies, in particular, that $D^{*}$ is symmetric with respect to the $x$-axis and convex in the $y$-direction. Let $C^{*}=\partial D^{*}$ be the boundary of $D^{*}$. Steiner used his symmetrization to show that

$$
\text { (a) area } D=\text { area } D^{*}, \quad \text { (b) length } C^{*} \leq \text { length } C \text {, }
$$

which implies the classical isoperimetric inequality

$$
\frac{\text { area } D}{(\text { length } C)^{2}} \leq \frac{1}{4 \pi}
$$

assuming the existence of a minimizer.

This ingenious idea of Steiner has appeared to be extremely fruitful and was exploited over the years by many authors, who proved numerous, so-called, isoperimetric inequalities for several important geometrical and physical quantities characterizing the

Date: September 25, 2018.

2000 Mathematics Subject Classification. Primary 58G35, 35A30, 35B05, 35B50, 35J60, 35K55; Secondary 28D05, 26D10.

Key words and phrases. Continuous symmetrization, Steiner symmetrization, rearrangement, polarization, integral inequality, boundary value problem, comparison theorem. 
shape of planar and solid regions. We want to mention the following four such inequalities, for the transfinite diameter $d(\bar{D})$ that is equal to the logarithmic capacity cap $(\bar{D})$, for the inner radius $r(D, a)$ of $D$ at its point $a \in D$, for the torsional rigidity $P(D)$, and for the principal frequency $\lambda(D)$ :

$$
\begin{array}{ll}
\text { (c) } d\left(\overline{D^{*}}\right) \leq d(\bar{D}), & \text { (d) } r\left(D^{*}, a^{*}\right) \geq r(D, a) \text { for every } a \in D, \\
\text { (e) } P\left(D^{*}\right) \geq P(D), & \text { (f) } \lambda\left(D^{*}\right) \leq \lambda(D) .
\end{array}
$$

The first period of history of symmetrization was summarized in the classical monograph of George Pólya and Gabor Szegö "Isoperimetric inequalities in mathematical physics" [24]. This fundamental study of isoperimetric inequalities was filled with new ideas, results, and problems, some of them still remain open. The story of continuous symmetrization also has its source in this book. The following question was raised in Note B of [24]:

Is it possible to define a transformation $T_{\lambda}$ on $D$ depending in a continuous way on the parameter $\lambda, 0 \leq \lambda \leq 1$, such that the following conditions are satisfied:

I. $T_{0}$ is the identity.

II. $T_{1}$ is the transformation replacing $C$ by the symmetrized curve $C^{*}$, that is, $T_{1}$ is Steiner's symmetrization with respect to the line $l$.

III. For every $\lambda, 0 \leq \lambda \leq 1, T_{\lambda}$ has the same effect as described under (a)-(d).

Although Pólya and Szegö mentioned only relations (a)-(d), a similar question about inequalities (e) and (f) falls into the same context.

The authors of [24] did not explain explicitly what motivated them to study this problem. Among obvious reasons we want to mention the following three:

- First, from the point of view of classical mechanics it is interesting to embed $D$ and $D^{*}$ into a continuous path, along which all basic geometrical and physical characteristics of shape vary continuously and monotonically.

- Often better estimates, than those provided by inequalities (b)-(f), are needed. Indeed, even for simple shapes, for example, for the rhombus having angle $\alpha=\pi / 100$ centered at $a=0$, the gap in each of the inequalities (b)-(f) exceeds $40 \%$.

- Steiner's symmetrization changes a given shape globally into a symmetric one. So, this transformation will not work in problems, where the minimizer does not possesses a global symmetry and in problems concerned with local minimality.

Pólya and Szegö themselves studied this problem. In collaboration with M. Schiffman [24, Note B], they presented one such continuous transformation and proved the relations (a)-(d) for the case of convex domains. Suppose that $D$ is a convex domain on $\mathbb{R}^{2}$ bounded from below and above by the graphs of functions $y=y_{1}(x)$ and $y=y_{2}(x)$, respectively, such that $y_{1}(x)<y_{2}(x)$ for all $a<x<b$. For a given continuous function $\phi:[\alpha, \beta] \rightarrow[0,1]$, let

$$
y_{1}^{t}=y_{1}-\phi(t) \frac{y_{1}+y_{2}}{2}, \quad y_{2}^{t}=y_{2}-\phi(t) \frac{y_{1}+y_{2}}{2}
$$

and

$$
D^{t, \phi}=\left\{(x, y) \in \mathbb{R}^{2}: a<x<b, y_{1}^{t}<y<y_{2}^{t}\right\}
$$


If $\phi$ is an increasing homeomorphism from $[\alpha, \beta]$ onto $[0,1]$, then it is clear that formulas (1.2), (1.3) define a continuous path from $D$ into $D^{*}$. Choosing $\phi(t)=t$, Pólya and Szegö [24] proved that the relations (a)-(d) hold true for all convex domains. S. Abramovich [1] used a variant of Pólya-Szegö's continuous symmetrization to prove monotonicity of eigenvalues of certain second order differential equations in one variable.

Another continuous transformation, again for smooth convex domains, was introduced by A. McNabb in 1967 [22]. His transformation, called the partial Steiner symmetrization, can be defined as follows. We quote from [22]:

" A partial Steiner symmetrization of $D$ may be performed in the following way. If the constant $\alpha$ lies between certain limits $\left(\alpha_{L}<\alpha<\alpha_{R}\right)$, the line $x=\alpha$ will intersect the curve defined by midpoints of the line segments composing $D$. If just those line segments which have their midpoints to the left of $x=\alpha$ are translated parallel to themselves until these central points lie on $x=\alpha$, the ends of the segments now define a curve $C_{\alpha}$ bounding a partially symmetrized region $D_{\alpha}$. It is as though the line $x=t$ swept across the $x-y$-plane from $t=-\infty$ to $t=\alpha$ and the midpoints of the line segments became attached to the line as it passed over them. As $t$ increases from $\alpha=\alpha_{L}$ to $\alpha=\alpha_{R}, D$ continuously evolves through a sequence $D_{t}$ of partially symmetrized regions to its Steiner symmetrization $D^{*}$."

As the author noted in [22], his goal was to demonstrate on simple examples how his transformation works. So, the treatment in [22] was heuristic and technical "difficulties were glossed over" there.

We also want to mention two interesting continuous transformations discovered in [31] and [23], but those are not related, at least not directly, to the problem raised in [24].

The first continuous transformation into Steiner symmetrization, which works for nonconvex domains and satisfies all the requirements of the Pólya-Szegö problem, was introduced by this author [27]. Our continuous symmetrization, which we will abbreviate as $S C$ symmetrization, can be considered as an extension of McNabb's partial Steiner symmetrization for the case of non-convex domains. ${ }^{1}$ We want to emphasize here that the approaches used in [22] and [27] are different.

It is interesting to mention that, eventually, the original idea of Pólya and Szegö was developed by F. Brock [12], [13], who defined a continuous symmetrization, called $B C$ symmetrization in this paper, which works for non-convex domains. This was achieved by choosing a parametrization $\phi(t)=1-e^{-t},-\infty<t<\infty$, in (1.2), (1.3), combined with some other innovations. Instead of abbreviations SC and BC, we sometimes write "Solynin's continuous symmetrization" and "Brock's continuous symmetrization", respectively. One particular difference between SC symmetrization and $\mathrm{BC}$ symmetrization is that under the first transformation the change of the shape is localized near some boundary arcs while the second transformation changes the boundary globally.

Although the exposition in [27] was given for planar domains, in the final Remark 5 [27], the author emphasized that all definitions and proofs can be extended without substantial changes to $n$-dimensional spaces and that all major results of the paper have $n$-dimensional counterparts.

\footnotetext{
${ }^{1}$ The paper [27] does not refer to McNabb's work [22] since at that time the author was not aware of McNabb's publication.
} 
The primary goal of the present paper is to give a full scale account of Solynin's continuous symmetrization in the $n$-dimensional setting. Since the paper [27] is practically unknown to the experts (its English translation is often inadequate, actually it looks like a computer translation), we want to mention here the major innovations introduced in [27]. First of all, the polarization was used for the first time in [27] in the context of continuous symmetrizations. Then, an analog of the semigroup property was applied to prove some results about the continuous SC symmetrization. Later on, F. Brock [13] used a similar property as a part of the definition of his continuous symmetrization. Uniqueness results were treated in [27] in all their generality. The latter leads, under certain conditions, to strict monotonicity of the domain characteristics under consideration as the functions of the parameter of symmetrization. Finally, the SC symmetrization was applied in [27] to prove local symmetry in some problems on Green's functions and harmonic measures. A similar approach to local symmetry in a more general context was also used in the papers [12] and [13].

This paper is organized as follows. Section 2 contains our basic notations. In particular, we introduce there necessary spaces of functions and classes of domains. In Section 3, we remind the reader of basic properties of the Steiner $(k, n)$-symmetrization and polarization. The exposition in Sections 2 and 3 follows the lines of our paper [14] joint with F. Brock. Sections 4-8 are devoted to geometric aspects of SC 1-symmetrization.

In Section 9, we give an inductive definition of the continuous $(k, n)$-Steiner symmetrization for any $2 \leq k \leq n$.

Sections 10 and 11, where we again follow the lines of the paper [14], contain our main applications. In Section 10, we show that many integral inequalities known for the Steiner symmetrization have their continuous counterparts for the continuous $(k, n)$ symmetrization as well. In Section 11, we give a similar treatment of the comparison theorems for solutions of some elliptic and parabolic PDE's. Many proofs in Sections 10 and 11 related to the $L^{p}$-classes and Sobolev classes are based on ideas suggested by F. Brock, when we worked on Sections 9 and 10 of our joint paper [14], and which he developed further in [13].

In the present paper we combined and extended the ideas and methods developed in [27], [28], and [14]. Preparing this article for publication, the author used his notes written in the Fall semester, 1995 during his stay at the Mathematisches Forschungsinstitut Oberwolfach under the financial support of Volkswagen-Stiftung, RiP-program for Friedemann Brock and Alexander Solynin. Our intention at that time was to present in a joint paper our results for both SC $k$-dimensional continuous symmetrization and BC $k$-dimensional continuous symmetrization. Since 1995, Brock's continuous symmetrization and its applications were already discussed in several publications. So, in this paper, we are concentrating on the Solynin's continuous symmetrization only. Although the original plan for this paper was changed, this work remains closely related to the paper [14] joint with F. Brock, where such a possible continuation was referenced as "An approach to continuous symmetrization via polarization".

\section{Preliminaries}

The following notations will be used throughout the paper. Let $\mathbb{R}^{n}$ be the Euclidean space, $\mathbb{R}_{+}^{n}=\left\{\left(x_{1}, \ldots, x_{n}\right) \in \mathbb{R}^{n}: x_{i}>0,1 \leq i \leq n\right\}$. 
For $A \subset \mathbb{R}^{n}$, let $\bar{A}$ and $\partial A$ denote the closure and the boundary of $A$, respectively. If $A, B \subset \mathbb{R}^{n}$ then $A+B:=\{z: z=x+y, x \in A, y \in B\}$ denotes the Minkowski sum of $A$ and $B$. For $x, y \in \mathbb{R}^{n}$, by $|x|$ and $\langle x, y\rangle$ we denote the norm of $x$ and the scalar product of $x$ and $y$, respectively. Then $H(a, n)$ and $\Sigma(a, n)$ will denote the half-space $\left\{x \in \mathbb{R}^{n}:\langle(x-a), n\rangle>0\right\}$ and the hyperplane $\left\{x \in \mathbb{R}^{n}:\langle(x-a), n\rangle=0\right\}$ defined by the point $a \in \mathbb{R}^{n}$ and the unit vector $n \in \mathbb{R}^{n}$. For $M \subset \mathbb{R}^{n}$, by $\mathcal{L}^{n}(M)$ we denote the $n$-dimensional Lebesgue measure of $M$. By $\mathcal{M}_{n}, \mathcal{F}_{n}$, and $\mathcal{G}_{n}$ we denote the sets of all measurable, compact, and open subsets of $\mathbb{R}^{n}$, respectively. Then $\mathcal{M}_{n, b}$ and $\mathcal{G}_{n, b}$ will denote collections of all bounded subsets of $\mathcal{M}_{n}$ and $\mathcal{G}_{n}$.

Generally, we treat measurable sets only in an a.e. sense, i.e. we write

$$
\begin{array}{lll}
M=N & \text { if and only if } & \mathcal{L}^{n}(M \triangle N)=0, \\
M \subset N & \text { if and only if } & \mathcal{L}^{n}(M \backslash N)=0 .
\end{array}
$$

By $B_{r}^{(n)}\left(x_{0}\right)$ we denote the open ball in $\mathbb{R}^{n}$ with radius $r>0$ centered at $x_{0}$ and we write $B_{r}^{(n)}=B_{r}^{(n)}(0), B^{(n)}=B_{r} 1^{(n)}$. If $A \subset \mathbb{R}^{n}$ and $\varepsilon>0$, then we denote by $A_{\varepsilon}:=A+\varepsilon \overline{B^{(n)}}$ the exterior parallel set of $A$. The Hausdorff distance between compact sets $A$ and $B$ is defined by

$$
d(A, B):=\inf \left\{\varepsilon>0: A \subset B_{\varepsilon}, B \subset A_{\varepsilon}\right\} .
$$

It is well known that $d$ is a metric on $\mathcal{F}_{n}$. We define, by the metric $d$, the convergence of a sequence of sets $F_{i} \in \mathcal{F}_{n}, i=1,2, \ldots$, to a set $F \in \mathcal{F}_{n}$ by

$$
\lim _{i \rightarrow \infty} F_{i}=F \quad \text { if and only if } d\left(F_{i}, F\right) \rightarrow 0 \text { as } i \rightarrow \infty \text {. }
$$

If $\Omega$ is an open set in $\mathbb{R}^{n}$ and $p \in[1, \infty]$ then $\|\cdot\|_{p}$ denotes the usual norm in the space $L^{p}(\Omega)$. For functions $u \in C\left(\mathbb{R}^{n}\right)$ we define the modulus of continuity by

$$
\omega_{u}(\delta):=\sup \{|u(x)-u(y)|:|x-y|<\delta\}, \quad \delta>0 .
$$

By $W^{1, p}(\Omega)$ we denote the Sobolev space of functions $u \in L^{p}(\Omega)$ having generalized partial derivatives $u_{x_{i}} \in L^{p}(\Omega), i=1, \ldots, n$, and we write

$$
\|u\|_{W^{1, p}(\Omega)}:=\|u\|_{p}+\sum_{i=1}^{n}\left\|u_{x_{i}}\right\|_{p}
$$

for the norm in this space. By $W_{0}^{1, p}(\Omega)$ we denote the completion of the set of infinitely differentiable functions with compact support in $\Omega$, denoted by $C_{0}^{\infty}(\Omega)$, under the norm (2.1). Usually we extend measurable functions $u: \Omega \rightarrow \mathbb{R}_{0}^{+}$by zero outside $\Omega$ so that $W_{0}^{1, p}(\Omega) \subset W^{1, p}\left(\mathbb{R}^{n}\right)$ in that sense. By $C_{0}^{0,1}(\Omega)$ we denote the space of Lipschitz functions with compact support in $\Omega$. For any function space the lower subscript "+" denotes the corresponding subspace of nonnegative functions, e.g. $L_{+}^{p}(\Omega), W_{0+}^{1, p}(\Omega)$, etc.

Let $\mathcal{S}_{n}$ denote the class of real measurable functions $u$ satisfying

$$
\mathcal{L}^{n}(\{u>c\})<\infty \text { for all } c>\inf u .
$$

Here and in the following we use the following abbreviation: $\{u>c\}=\left\{x \in \mathbb{R}^{n}\right.$ : $u(x)>c\}$. Note that the spaces $L_{+}^{p}\left(\mathbb{R}^{n}\right), C_{0+}^{0,1}\left(\mathbb{R}^{n}\right)$, and the space $W_{+}^{1, p}\left(\mathbb{R}^{n}\right)$ with 
$1 \leq p<\infty$ are subspaces of $\mathcal{S}_{n+}$. The space of measurable functions with bounded variation is denoted by $B V\left(\mathbb{R}^{n}\right)$ and we write

$$
\|D u\|_{B V}:=\sup \left\{\int_{\mathbb{R}^{n}} u \sum_{i=1}^{n} \frac{\partial \psi_{i}}{\partial x_{i}} d x: \sum_{i=1}^{n} \psi_{i}^{2} \leq 1, \psi_{i} \in C_{0}^{\infty}\left(\mathbb{R}^{n}\right), i=1, \ldots, n\right\} .
$$

Recall also that if $u \in W^{1,1}\left(\mathbb{R}^{n}\right)$, then $\|D u\|_{B V}=\|\nabla u\|_{1}$. Furthermore, if $M$ is a Caccioppoli set in $\mathbb{R}^{n}$, then $\|D \chi(M)\|_{B V}$ is the perimeter of $M$ in the sense of De Giorgi, see [32].

Finally, a function $j: \mathbb{R}_{0}^{+} \rightarrow \mathbb{R}_{0}^{+}$is called a Young function if $j$ is continuous and convex with $j(0)=0$.

\section{Steiner SYMmetrization AND POLARIZATION}

First we discuss some general properties of rearrangements. We remind the reader that a set transformation $T$ (defined on $\mathcal{M}_{n}$ ) is called a rearrangement if it is monotone and measure preserving, i.e. if $T(A) \subset T(B)$ for all $A$ and $B$ such that $A \subset B$ and $\mathcal{L}^{n}(T(A))=\mathcal{L}^{n}(A)$ for every measurable set $A$.

The class $\mathcal{S}_{n}$ introduced in the previous section is the natural class of functions for which a rearrangement can be defined. If $T$ is a rearrangement and $u$ is in $\mathcal{S}_{n}$, then the relations

$$
T u(x):=\operatorname{ess} \sup \{c>\inf u: x \in T(\{u>c\})\} \quad \inf T u:=\inf u,
$$

define a function $T u$ on $\mathbb{R}^{n}$. If $u \in \mathcal{S}_{n}$ is continuous, then "ess sup" in (3.1) can be replaced by "sup". Clearly the function $T u$ is uniquely determined almost everywhere. Since $T$ is measure preserving,

$$
\mathcal{L}^{n}(T(\{u>c\}))=\mathcal{L}^{n}(\{T u>c\}) \quad \text { for all } c>\inf u .
$$

Thus $T u \in \mathcal{S}_{n}$ if $u \in \mathcal{S}_{n}$. The mapping $T: \mathcal{S}_{n} \rightarrow \mathcal{S}_{n}$ constructed in this way is again called a rearrangement. The following non-expansivity lemma will be very useful in Sections 10 and 11, see [14, Theorem 3.1].

Lemma 3.1. Let $T$ be a rearrangement. Then for every Young function $j$, we have

$$
\int_{\mathbb{R}^{n}} j(|T u-T v|) d x \leq \int_{\mathbb{R}^{n}} j(|u-v|) d x \quad \text { for all } u, v \in \mathcal{S}_{n},
$$

whenever either one of the integrals in (3.2) converges.

Sometimes we will say that two functions $u, v \in \mathcal{S}_{n}$ are rearrangements of each other if inf $u=\inf v$ and $\mathcal{L}^{n}(\{u>c\})=\mathcal{L}^{n}(\{v>c\})$ for all $c>\inf u$.

We will also use some additional properties of rearrangements. A set transformation $T$ is called open or compact if $T(A)$ is open or compact whenever $A$ is of the same kind, respectively. We say that $T$ is continuous from the inside if $\cup_{i} T\left(G_{i}\right)=T\left(\cup_{i} G_{i}\right)$ for every increasing sequence $\left\{G_{i}\right\} \subset \mathcal{G}$. Similarly we say that $T$ is continuous from the outside if $\cap_{i} T\left(F_{i}\right)=T\left(\cap_{i} F_{i}\right)$ for every decreasing sequence $\left\{F_{i}\right\} \subset \mathcal{F}$.

Finally, a rearrangement $T$ is called smoothing if $T\left(F_{r}\right) \supset(T(F))_{r}$ for every $F \in \mathcal{F}$ and $r>0$. Smoothing rearrangements were introduced by Sarvas [25].

Let us now recall the definitions of the $(k, n)$-Steiner symmetrizations (for further information see [30], [21], and [25]). 
Definition 3.1. Every $(n-k)$-dimensional plane $\Sigma \subset \mathbb{R}^{n}$ with $1 \leq k \leq n$ defines a $(k, n)$-Steiner symmetrization $S$ as follows:

For every $x \in \Sigma$ let $\Lambda(x)$ denote the $k$-dimensional plane through $x$ and orthogonal to $\Sigma$.

1) Let $M \in\left(\mathcal{F}_{n} \cup \mathcal{G}_{n}\right) \cap \mathcal{M}_{n}$. If $\mathcal{L}^{k}(M \cap \Lambda(x))=0$, then $S(M) \cap \Lambda(x)$ is empty or the point $\{x\}$ according to whether $M \cap \Lambda(x)$ is empty or nonempty. If $\mathcal{L}^{k}(M \cap \Lambda(x))>0$, then

$$
S(M) \cap \Lambda(x)= \begin{cases}B_{r}(x) \cap \Lambda(x) & \text { if } M \text { is open, } \\ B_{r}(x) \cap \Lambda(x) & \text { if } M \text { is compact, }\end{cases}
$$

where $r>0$ is defined by the condition $\mathcal{L}^{k}\left(B_{r}(x) \cap \Lambda(x)\right)=\mathcal{L}^{k}(M \cap \Lambda(x))$.

2) Let $M \in \mathcal{M}_{n}$ where $M$ is neither open nor compact. Then the sets $S(M) \cap \Lambda(x)$ are defined in an a.e. sense by either one of the equations in (3.3).

From Definition 3.1 one deduces immediately that the $(k, n)$-Steiner symmetrization is a rearrangement which is continuous from the inside and from the outside. Note also that in case 2) Fubini's Theorem implies that the sets $M \cap \Lambda(x)$ are measurable with finite $\mathcal{L}^{k}$-measure for a.e. $x \in \Sigma$.

The $(n, n)$-Steiner symmetrization is often called the Schwarz symmetrization or the symmetric decreasing rearrangement, and we will denote it by $S^{\star}$.

For our purposes it will often be helpful to use a special coordinate system in $\mathbb{R}^{n}=$ $\mathbb{R}^{m} \times \mathbb{R}^{k}$, where $1 \leq k \leq n, m=n-k$ and

$$
x=\left(x_{1}, \ldots, x_{n}\right)=\left(x^{\prime}, y\right), \quad x^{\prime}=\left(x_{1}, \ldots, x_{n-k}\right), \quad y=\left(x_{n-k+1}, \ldots, x_{n}\right),
$$

in which the plane $\Sigma$ of symmetry becomes simply $\{y=0\}$. If $M \in \mathcal{M}_{n}$, we introduce the " $k$-slices" of $M$ at $x^{\prime}$ by

$$
M\left(x^{\prime}\right)=\left\{y \in \mathbb{R}^{k}:\left(x^{\prime}, y\right) \in M\right\}, \quad x^{\prime} \in \mathbb{R}^{n-k} .
$$

For instance, if $x^{\prime} \in \mathbb{R}^{n-k}$ with $1 \leq k<n$, then $B_{r}^{(n)}\left(x^{\prime}\right)$ will denote the $k$-slice of the ball $B_{r}^{(n)}$ at $x^{\prime}$ and not the ball in $\mathbb{R}^{n}$ centered at $x^{\prime}$. Let $S^{\star}\left(M\left(x^{\prime}\right)\right)$ denote the Schwarz symmetrization of $M\left(x^{\prime}\right)$, taken in $\mathbb{R}^{k}$. Then (3.3) reads

$$
S(M):=\left\{x=\left(x^{\prime}, y\right): y \in S^{\star}\left(M\left(x^{\prime}\right)\right), x^{\prime} \in \mathbb{R}^{n-k}\right\} .
$$

If $u \in \mathcal{S}_{n}$, then we obtain from (3.3) that the $(k, n)$-Steiner symmetrization $\mathcal{S} u$ of $u$ is given by the relations

$$
\mathcal{S} u\left(x^{\prime}, y\right)=\sup \left\{c>\inf u: x \in S\left(\left\{u\left(x^{\prime}, \cdot\right)>c\right\}\right)\right\} .
$$

(Here and in the following for simplicity $\left\{u\left(x^{\prime}, \cdot\right)>c\right\}$ denotes $\left\{y \in \mathbb{R}^{k}: u\left(x^{\prime}, y\right)>c\right\}$.) Let us mention again that the equations (3.4) and (3.5) have to be understood in the pointwise sense if and only if $u$ is continuous. Note also that $\mathcal{S} u$ is "radially symmetric and decreasing in $|y|$ ", i.e.

$$
\mathcal{S} u\left(x^{\prime}, y\right)=\mathcal{S} u\left(x^{\prime}, z_{1}\right) \geq \mathcal{S} u\left(x^{\prime}, z_{2}\right) \quad \text { if }|y|=\left|z_{1}\right| \leq\left|z_{2}\right|,
$$

where $x^{\prime} \in \mathbb{R}^{n-k}$ and $y, z_{1}, z_{2} \in \mathbb{R}^{k}$.

Sometimes we will write $S(M)=M^{*}$ and $\mathcal{S} u=u^{*}$ for the symmetrized objects.

There is an approach due to Schwartz and Blaschke (see, for instance, [11]) reducing a $k$-dimensional symmetrization to $(k-1)$-dimensional symmetrizations, see Theorem 4.32 in [25]. We will use a slightly refined version of this theorem. 
Let $S$ be a $(k, n)$-Steiner symmetrization in $\mathbb{R}^{n}=\mathbb{R}^{m} \times \mathbb{R}^{k}$ with the symmetry plane $\Sigma=\{y=0\}$. Let $\vec{v}_{1}$ and $\vec{v}_{2}$ be unit vectors orthogonal to $\Sigma$ which form an angle $\gamma \pi$, where $\gamma \in(0,1)$ is irrational. Let $S_{i}$ be the $(k-1, n)$-Steiner symmetrization with the symmetry plane $\Sigma_{i}$ defined by the plane $\Sigma$ and the unit vector $\vec{v}_{i}, i=1,2$.

For positive integer $j$ and $\Omega \in \mathcal{F}_{n} \cup \mathcal{G}_{n, b}$, let

$$
\begin{gathered}
\Omega_{j}=\left(S_{2} \circ S_{1}\right)^{m}(\Omega) \quad \text { if } j=2 m \text { is even, } \\
\Omega_{j}=S_{1} \circ\left(S_{2} \circ S_{1}\right)^{m}(\Omega) \quad \text { if } j=2 m+1 \text { is odd. }
\end{gathered}
$$

Here $\left(S_{2} \circ S_{1}\right)^{0}$ is the identity transformation.

Theorem 3.1. Let $S, S_{1}$, and $S_{2}$ be the symmetrizations defined above and let $\Omega^{*}=$ $S(\Omega)$. Then

$$
\begin{gathered}
\lim _{j \rightarrow \infty} d\left(\Omega_{j}, \Omega^{*}\right)=0 \quad \text { for every compact set } \Omega \in \mathcal{F}_{n}, \\
\lim _{j \rightarrow \infty} d\left(\partial \Omega_{j}, \partial \Omega^{*}\right)=0 \quad \text { for every bounded open set } \Omega \in \mathcal{G}_{n, b},
\end{gathered}
$$

and

$$
\lim _{j \rightarrow \infty} \mathcal{L}^{k}\left(\left(\Omega_{j}\left(x^{\prime}\right) \triangle \Omega^{*}\left(x^{\prime}\right)\right)=0\right.
$$

for every $\Omega \in \mathcal{F}_{n} \cup \mathcal{G}_{n, b}$ and every $x^{\prime} \in \mathbb{R}^{n-k}$.

For compact sets this theorem is a part of Theorem 4.32 in [25]. For bounded open sets the proof will be given in the Appendix. In Section 9, we will use the approximation scheme of Theorem 3.1 to give an inductive definition of our continuous $(k, n)$-Steiner symmetrization.

During the last decade we have seen increase of activity in the theory of symmetrization, partly triggered by the paper [14], that is related to the polarization. This simplest rearrangement was introduced for sets by V. Wolontis [33] in 1952 who attributed some ideas of his paper to L. Ahlfors. Ahlfors himself used polarization in [2], where he introduced this transformation for functions. The term polarization was suggested by V. N. Dubinin [15].

Let $\Sigma$ be a hyperplane in $\mathbb{R}^{n}$ and let $H$ be one of the open halfspaces into which $\mathbb{R}^{n}$ is divided by $\Sigma$. Let $\sigma_{H}$ denote the reflection in $\Sigma$. We write $\bar{x}=\sigma_{H}(x)$ for points $x \in \mathbb{R}^{n}$ and $\sigma_{H}(u)=u(\bar{x})$ for all $x \in \mathbb{R}^{n}$ for functions $u \in \mathcal{S}$.

Definition 3.2. If $u \in \mathcal{S}_{n}$, then its polarization $P u$ with the polarizer $H$ is given by

$$
P u(x):= \begin{cases}\max \{u(x), u(\bar{x})\} & \text { if } x \in H, \\ \min \{u(x), u(\bar{x})\} & \text { if } x \in \mathbb{R}^{n} \backslash H .\end{cases}
$$

If $M \in \mathcal{M}_{n}$, then the polarization $P(M)$ is given by its characteristic function via (3.12), i.e.

$$
\chi(P(M)):=P(\chi(M)) .
$$

In the case that $u$ is continuous and $M$ is open or closed, equations (3.12) and (3.13) have to be understood in the pointwise sense.

Equations (3.12) and (3.13) can also be written in the following more precise form

$$
P(M)=\left(\left(M \cup \sigma_{H}(M)\right) \cap H\right) \cup\left(M \cap \sigma_{H}(M)\right), \quad M \in \mathcal{M}_{n},
$$


and

$$
P u(x)=\operatorname{ess} \sup \{c>\inf u: x \in P(\{u>c\})\}, \quad x \in \mathbb{R}^{n}, \quad u \in \mathcal{S}_{n} .
$$

Of course, if $u \in \mathcal{S}_{n}$ is continuous, then "ess sup" in (3.15) can be replaced by "sup". From the representations (3.12)-(3.15) we see that the polarization $P$ is an open and compact rearrangement which is continuous from the inside and from the outside.

For the sake of simplicity, we will often use the subscript " $H$ " to denote any one of the polarized objects, i.e. we write $u_{H}$ and $M_{H}$ for $P u$ and $P(M)$, respectively.

It is worth mentioning, that the polarization of a connected set is not necessarily connected and may contain one multiply connected component.

There are three major approaches to polarization. The first one initiated in [33], [2], and [7] uses convolution type inequalities. In the most powerful and general form this approach culminated in Baernstein's fundamental work [6].

The second approach was introduced by V. N. Dubinin [15] who used the following representation of polarized functions. Let $v(x)=u(\bar{x}), w(x)=u_{H}(\bar{x}), x \in H$ with $x \in H$. Then

$$
u_{H}(x)=(u(x)-v(x))_{+} \quad \text { and } \quad w(x)=u(x)-(u(x)-v(x))_{+} .
$$

The latter under certain conditions leads to

$$
\begin{aligned}
\nabla u_{H}(x) & = \begin{cases}\nabla u(x) & \text { a.e. on }\{u>v\} \cap H, \\
\nabla v(x) & \text { a.e. on }\{u \leq v\} \cap H,\end{cases} \\
\nabla w(x) & = \begin{cases}\nabla v(x) & \text { a.e. on }\{u>v\} \cap H, \\
\nabla u(x) & \text { a.e. on }\{u \leq v\} \cap H,\end{cases}
\end{aligned}
$$

which easily leads to several Dirichlet type inequalities, see [14, Lemma 5.3].

The third approach suggested by A. Solynin [28] and developed further in [14] rests on the direct application of the maximum principle to prove comparison theorems for solutions of two related boundary value problems for certain partial differential equations defined in a given domain $\Omega$ and in the polarized domain $\Omega_{H}$. This approach will be used in Section 11 .

\section{Continuous $(1, n)$-Steiner symmetrization}

First we define a continuous transformation on $\mathbb{R}$. For $M \in \mathcal{M}_{1}$ and $-\infty<t \leq \infty$, the measuring function of $M$ is defined by

$$
m_{M}(t)=\mathcal{L}^{1}((-\infty, t) \cap M) .
$$

This definition shows that $m_{M}(t)$ is nondecreasing and Lipschitz continuous with constant 1:

$$
0 \leq m_{M}\left(t_{2}\right)-m_{M}\left(t_{1}\right) \leq t_{2}-t_{1} \text { for all } t_{1} \leq t_{2} .
$$

This implies that, for every $t \in \mathbb{R}$, the equation

$$
y-(1 / 2) m_{M}(y)=t
$$

has a unique solution $y=y_{M}(t) \in[t, \infty)$. The function $y=y_{M}(t)$, called the separating function of $M$, will play an important role in this study. Two basic properties of $y_{M}(t)$ given by the following lemma are immediate consequences of the above definitions and inequalities (4.2). 
Lemma 4.1. (a) If $M \subset N$, then $y_{M}(t) \leq y_{N}(t)$ for all $t \in \mathbb{R}$.

(b) If $t_{1}<t_{2}$, then

$$
t_{2}-t_{1} \leq y_{M}\left(t_{2}\right)-y_{M}\left(t_{1}\right) \leq 2\left(t_{2}-t_{1}\right)
$$

For $t \in \mathbb{R}$, the $T^{t}$-transformation of $M$ is defined as

$$
\begin{aligned}
& M^{t}=\left(y_{M}(t)-m_{M}(t), y_{M}(t)\right) \cup\left(M \cap\left[y_{M}(t), \infty\right)\right) \text { if } M \text { is open, } \\
& M^{t}=\left[y_{M}(t)-m_{M}(t), y_{M}(t)\right] \cup\left(M \cap\left[y_{M}(t), \infty\right)\right) \text { if } M \text { is compact. }
\end{aligned}
$$

If $M \in \mathcal{M}_{n}$ but is neither open or compact then $M^{t}$ is defined in the a.e. sense by either one of the equations (4.5) or (4.6).

Definition 4.1. The family of mappings $T^{t}: \mathcal{M}_{1} \rightarrow \mathcal{M}_{1}, t \in \mathbb{R}$, defined by $T^{t}(M)=$ $M^{t}$ is called the continuous symmetrization on $\mathbb{R}$.

Now we turn to $\mathbb{R}^{n}=\mathbb{R}^{n-1} \times \mathbb{R}$. For $\Omega \in \mathcal{M}_{n}$ and $x^{\prime} \in \mathbb{R}^{n-1}$, let $\Omega\left(x^{\prime}\right)=\{y \in \mathbb{R}$ : $\left.\left(x^{\prime}, y\right) \in \Omega\right\}$ be the 1 -slice of $\Omega$ at $x^{\prime}$. If $\Omega \in \mathcal{M}_{n}$ is open or compact then $\Omega^{\prime}$ will denote the orthogonal projection of $\Omega$ onto $\mathbb{R}^{n-1}$. Otherwise we put $\Omega^{\prime}=\left\{x^{\prime} \in \mathbb{R}^{n-1}\right.$ : $\left.\mathcal{L}^{1}\left(\Omega\left(x^{\prime}\right)\right) \neq 0\right\}$. The measuring function of $\Omega$ is defined by

$$
m_{\Omega}\left(x^{\prime}, t\right)=\left\{\begin{array}{cl}
m_{\Omega\left(x^{\prime}\right)}(t) & \text { if } x^{\prime} \in \Omega^{\prime} \\
0 & \text { otherwise. }
\end{array}\right.
$$

One can easily show that $m_{\Omega}\left(x^{\prime}, t\right)$ is lower semicontinuous in $x^{\prime}$ if $\Omega$ is open and it is upper semicontinuous in $x^{\prime}$ if $\Omega$ is compact.

Definition 4.2. The function $y_{\Omega}: \mathbb{R}^{n-1} \times \mathbb{R} \rightarrow \mathbb{R}$ defined by

$$
y_{\Omega}\left(x^{\prime}, t\right)=\left\{\begin{array}{cl}
y_{\Omega\left(x^{\prime}\right)}(t) & \text { if } x^{\prime} \in \Omega^{\prime} \\
t & \text { otherwise }
\end{array}\right.
$$

is called the separating function and the graph $F_{\Omega}(t)=\left\{\left(x^{\prime}, y_{\Omega}\left(x^{\prime}, t\right)\right): x^{\prime} \in \mathbb{R}^{n-1}\right\}$ is called the frontier of symmetrization of $\Omega$ at $t$.

To simplify notation we will skip the symbol of the set if its meaning is clear from the context. Thus we often write $m\left(x^{\prime}, t\right), y\left(x^{\prime}, t\right)$, etc. instead of $m_{\Omega}\left(x^{\prime}, t\right), y_{\Omega}\left(x^{\prime}, t\right)$, etc.

Lemma 4.2. For a fixed $t \in \mathbb{R}$ and $\Omega \in \mathcal{M}_{n}$, the separating function $y\left(x^{\prime}, t\right)$ is lower semicontinuous on $\mathbb{R}^{n-1}$ if $\Omega$ is open and it is upper semicontinuous on $\mathbb{R}^{n-1}$ if $\Omega$ is compact.

Proof. Let $\Omega$ be open and $x_{0}^{\prime} \in \mathbb{R}^{n-1}$. If $m\left(x_{0}^{\prime}, t\right)=0$, then $y\left(x^{\prime}, t\right) \geq t=y\left(x_{0}^{\prime}, t\right)$ for all $x^{\prime}$ and the lower semi-continuity follows.

Assume that $y\left(x^{\prime}, t\right)$ is not lower semicontinuous at $x_{0}^{\prime}$ such that $m\left(x_{0}^{\prime}, t\right)>0$. Then for some $\delta>0$ and some sequence $x_{k}^{\prime} \rightarrow x_{0}^{\prime}$,

$$
y\left(x_{k}^{\prime}, t\right) \leq y\left(x_{0}^{\prime}, t\right)-\delta, \quad k=1,2, \ldots
$$

From (4.3), (4.2), and (4.9) we obtain

$$
\begin{aligned}
m\left(x_{k}^{\prime}, y\left(x_{0}^{\prime}, t\right)-\delta\right)-2\left(y\left(x_{k}^{\prime}, t\right)-t\right) & =m\left(x_{k}^{\prime}, y\left(x_{0}^{\prime}, t\right)-\delta\right)-m\left(x_{k}^{\prime}, y\left(x_{k}^{\prime}, t\right)\right) \\
& \leq y\left(x_{0}^{\prime}, t\right)-y\left(x_{k}^{\prime}, t\right)-\delta .
\end{aligned}
$$

This combined with (4.9) gives

$$
\limsup _{k \rightarrow \infty} m\left(x_{k}^{\prime}, y\left(x_{0}^{\prime}, t\right)-\delta\right) \leq 2\left(y\left(x_{0}^{\prime}, t\right)-t-\delta\right) .
$$


Since $\Omega$ is open the function $m\left(x^{\prime}, t\right)$ is lower semicontinuous in $x^{\prime}$. Hence

$$
\liminf _{k \rightarrow \infty} m\left(x_{k}^{\prime}, y\left(x_{0}^{\prime}, t\right)-\delta\right) \geq m\left(x_{0}^{\prime}, y\left(x_{0}^{\prime}, t\right)-\delta\right) .
$$

Using (4.2) and (4.3) we obtain

$$
m\left(x_{0}^{\prime}, y\left(x_{0}^{\prime}, t\right)-\delta\right) \geq m\left(x_{0}^{\prime}, y\left(x_{0}^{\prime}, t\right)\right)-\delta=2\left(y\left(x_{0}^{\prime}, t\right)-t\right)-\delta
$$

Now (4.12) and (4.13) yield the inequality

$$
\liminf _{k \rightarrow \infty} m\left(x_{k}^{\prime}, y\left(x_{0}^{\prime}, t\right)-\delta\right) \geq 2\left(y\left(x_{0}^{\prime}, t\right)-t\right)-\delta,
$$

which contradicts (4.11). This proves Lemma 4.2 for the case of open sets. If $\Omega$ is compact the proof is similar and is left to the reader.

Now we are ready to define what the 1-dimensional continuous symmetrization is.

Definition 4.3. A family of set transformations $T^{t}: \mathcal{M}_{n} \rightarrow \mathcal{M}_{n}, t \in \mathbb{R}$, defined by

$$
T^{t}(\Omega)=\Omega^{t}:=\left\{\left(x^{\prime}, y\right): x^{\prime} \in \Omega^{\prime}, y \in \Omega^{t}\left(x^{\prime}\right)\right\}
$$

will be called a continuous 1-symmetrization or SC1-symmetrization. Any single transformation of this family will be called a $T^{t}$-transformation. For $t=-\infty$, we define $T^{-\infty}$ to be the identity transformation.

The set $\Omega^{t}$ itself will be called the $T^{t}$-transformation of $\Omega$ or the partial symmetrization of $\Omega$ with respect to the plane $\{y=t\}$. Sometimes we will refer to this plane $\{y=t\}$ as the moving plane of symmetrization. Accordingly, the parameter $t$ will be called the height of the moving plane.

The frontier of symmetrization $F_{\Omega}(t)$ divides $\mathbb{R}^{n}$ into two parts

$$
H_{+}(t)=\left\{\left(x^{\prime}, y\right): y>y\left(x^{\prime}, t\right)\right\} \quad \text { and } \quad H_{-}=\left\{\left(x^{\prime}, y\right): y<y\left(x^{\prime}, t\right)\right\}
$$

called the upper and lower subspaces of symmetrization, respectively. Let

$$
\Omega_{-}(t)=\Omega \cap H_{-}(t), \quad \Omega_{+}(t)=\Omega \backslash H_{-}(t) \quad \text { if } \Omega \text { is open }
$$

and

$$
\Omega_{+}(t)=\Omega \cap H_{+}(t), \quad \Omega_{-}(t)=\Omega \backslash H_{+}(t) \quad \text { if } \Omega \text { is compact. }
$$

If $\Omega$ is neither open or compact then $\Omega_{-}(t)$ and $\Omega_{+}(t)$ are defined a.e. by (4.17).

In Lemma 4.3 below we list some useful properties of SC symmetrization, which follow directly from the above definitions. By $\Omega_{-}^{*}(t)$ we denote the $(1, n)$-Steiner symmetrization of $\Omega_{-}(t)$ with respect to $\{y=t\}$.

Lemma 4.3. (a) $\Omega^{t}=\Omega_{-}^{*}(t) \cup \Omega_{+}(t)$ for all $t \in \mathbb{R}$ and every $\Omega \in \mathcal{M}_{n}$.

(b) $\mathcal{L}^{1}\left(\Omega^{t}\left(x^{\prime}\right)\right)=\mathcal{L}^{1}\left(\Omega\left(x^{\prime}\right)\right)$ for all $x^{\prime} \in \mathbb{R}^{n-1}$ and $\mathcal{L}^{n}\left(\Omega^{t}\right)=\mathcal{L}^{n}(\Omega)$.

(c) If $\Omega$ is open, then $\Omega_{-}(t), \Omega_{-}^{*}(t)$, and $\Omega^{t}$ are open sets and $\Omega_{-}^{*}(t) \cap\{y \geq t\}=H_{-}(t) \cap\{y \geq t\}$.

(d) If $\Omega$ is compact, then $\Omega_{-}(t), \Omega_{-}^{*}(t)$, and $\Omega^{t}$ are compact sets and $\Omega_{-}^{*}(t) \cap\{y \geq t\}=\{y \geq t\} \backslash H_{+}(t)$.

We note that Lemma 4.3(a) gives an equivalent definition of $\Omega^{t}$ and Lemma 4.3(b) shows that the $T^{t}$-transformation preserves measures.

Now we will show that SC symmetrization possesses all basic geometric properties of classical symmetrizations. 
Lemma 4.4. For a fixed $t \in \mathbb{R}$, the $T^{t}$-transformation is an open, compact, and smoothing rearrangement, which is continuous from the inside and from the outside.

Proof. Lemma 4.1(a) and Definition 4.3 imply that $T^{t}(M) \subset T^{t}(N)$ if $M \subset N$ and therefore the $T^{t}$-transformation is monotone. Since, by Lemma 4.3(b), the $T^{t}$ transformation preserves the measure of sets, it follows that the $T^{t}$-transformation is a rearrangement.

From Definition 4.3 and Lemma 4.3(c) and (d), one can easily verify that the $T^{t}$ transformation is continuous from the inside and from the outside.

To prove that the $T^{t}$-transformation is smoothing, we fix a compact set $K$ and $\varepsilon>0$. Since, by Lemma $4.3(\mathrm{a}), K^{t}=K_{-}^{*}(t) \cup \overline{K_{+}(t)}$, we have

$$
K^{t}+\varepsilon \overline{B^{(n)}}=\left(K_{-}^{*}(t)+\varepsilon \overline{B^{(n)}}\right) \cup\left(\overline{K_{+}(t)}+\varepsilon \overline{B^{(n)}}\right) .
$$

Using Lemma 4.3(d) and the smoothing property of the Steiner symmetrization we obtain

$$
T^{t}\left(K+\varepsilon \overline{B^{(n)}}\right) \supset T^{t}\left(K_{-}(t)+\varepsilon \overline{B^{(n)}}\right)=\left(K_{-}(t)+\varepsilon \overline{B^{(n)}}\right)^{*} \supset K_{-}^{*}(t)+\varepsilon \overline{B^{(n)}},
$$

where $(\cdot)^{*}$ denotes the Steiner symmetrization with respect to $\{y=t\}$. Since $K_{+}(t)$ remains unchanged under the $T^{t}$-transformation of $K$ one can easily see that

$$
T^{t}\left(K+\varepsilon \overline{B^{(n)}}\right) \supset T^{t}\left(K_{+}(t)+\varepsilon \overline{B^{(n)}}\right)=\overline{K_{+}(t)}+\varepsilon \overline{B^{(n)}} .
$$

Now $(4.18)-(4.20)$ yield:

$$
T^{t}\left(K+\varepsilon \overline{B^{(n)}}\right) \supset T^{t}(K)+\varepsilon \overline{B^{(n)}},
$$

which shows that the $T^{t}$-transformation is smoothing.

It was shown by Sarvas, see Lemmas 3.2 and 3.3 in [14], that the properties established above imply that the $T^{t}$-transformation is open and compact. The proof is complete.

Since for any fixed $t \in \mathbb{R}$, the $T^{t}$-transformation is a rearrangement of sets there is a standard way to define a corresponding transformation of functions:

Definition 4.4. SC 1-symmetrization of functions: Let $u \in \mathcal{S}_{n}$. Then the family of functions $u^{t}, t \in \mathbb{R}$, defined for $x \in \mathbb{R}^{n}$ by

$$
u^{t}(x):= \begin{cases}\operatorname{ess} \sup \left\{c>\inf u: x \in T^{t}(\{u>c\})\right\} & \text { if } x \in \bigcup_{c>\inf u} T^{t}(\{u>c\}) \\ \inf u & \text { if } x \notin \bigcup_{c>\inf u} T^{t}(\{u>c\})\end{cases}
$$

is called the SC 1-symmetrization of $u$.

\section{Continuity properties of SC symmetrization}

Now we prove that the $T^{t}$-transformation depends continuously on the parameter $t$, which justifies the use of the word "continuous" in the name of this transformation. 
Lemma 5.1. Let $t_{k}, k=1,2, \ldots$ be an increasing sequence such that $t_{k} \rightarrow t^{\prime}<\infty$ and let $\Omega^{\prime}=\Omega^{t^{\prime}}$ and $\Omega_{k}=\Omega^{t_{k}}$. Then

$$
d\left(\partial \Omega_{k}, \partial \Omega^{\prime}\right) \rightarrow 0 \quad \text { as } \quad k \rightarrow \infty
$$

if $\Omega$ is an open bounded set and

$$
d\left(\Omega_{k}, \Omega^{\prime}\right) \rightarrow 0 \quad \text { as } \quad k \rightarrow \infty
$$

if $\Omega$ is a compact set.

Proof. We will prove the lemma for open sets. The proof for compact sets follows the same lines, it is easier and is left to the reader.

If (5.1) does not hold, then there exist subsequences of the sequences $t_{k}$ and $\Omega_{k}$, which we still call $t_{k}$ and $\Omega_{k}$, such that

$$
d\left(\partial \Omega_{k}, \partial \Omega^{\prime}\right) \geq \varepsilon, \quad k=1,2, \ldots
$$

for some $\varepsilon>0$. Since $\Omega$ is bounded equation (5.3) implies that there are subsequences (again denoted by $t_{k}$ and $\Omega_{k}$ ) and a sequence of points $x_{k} \in \mathbb{R}^{n}$ with $x_{k} \rightarrow x_{0} \in \mathbb{R}^{n}$ such that one of the following two conditions is satisfied:

(a) $x_{k} \in \partial \Omega^{\prime}$ and $\operatorname{dist}\left(x_{k}, \partial \Omega_{k}\right) \geq \varepsilon$ for all $k=0,1,2, \ldots$

(b) $x_{k} \in \partial \Omega_{k}$ for $k=1,2, \ldots$ and $\operatorname{dist}\left(x_{k}, \partial \Omega^{\prime}\right) \geq \varepsilon$ for $k=0,1,2, \ldots$

The case (a) contradicts Lemma 5.2 below, which characterizes the stability property of the boundary of $\Omega^{t}$.

Now we consider the case (b). Let

$$
x_{k}=\left(x_{k}^{\prime}, y_{k}\right) \rightarrow\left(x_{0}^{\prime}, y_{0}\right)=x_{0} .
$$

Then

$$
x_{k} \in \partial\left(\Omega_{-}^{*}\left(t_{k}\right)\right) \cup \partial\left(\Omega_{+}\left(t_{k}\right)\right) .
$$

Taking a subsequence if necessary we may restrict ourselves to two cases:

(1) In the first case we assume that $x_{k} \in \partial \Omega_{+}\left(t_{k}\right)$ for all $k=1,2, \ldots$. Since $x_{k} \notin \Omega_{k}$ we have $x_{k} \notin \Omega_{+}\left(t_{k}\right)$. Now if $x_{k} \in \Omega$ then $x_{k} \in \Omega_{-}\left(t_{k}\right)$. Since $\Omega_{-}\left(t_{k}\right)$ is open and $\Omega_{-}\left(t_{k}\right) \cap \Omega_{+}\left(t_{k}\right)=\emptyset$ we conclude that $x_{k} \notin \partial \Omega_{+}\left(t_{k}\right)$, which contradicts the above assumption. Thus in the case under consideration $x_{k} \notin \Omega$ and therefore $x_{k} \in \partial \Omega$ for all $k=1,2, \ldots$. Taking the limit we get

$$
x_{0} \in \partial \Omega .
$$

Since $x_{k} \in \partial \Omega_{+}\left(t_{k}\right)$ using (4.4) we obtain

$$
y_{k} \geq y\left(x_{k}^{\prime}, t_{k}\right) \geq y\left(x_{k}^{\prime}, t^{\prime}\right)-2\left(t^{\prime}-t_{k}\right) .
$$

Passing to the limit we get

$$
y_{0} \geq y\left(x_{0}^{\prime}, t^{\prime}\right)
$$

since $y\left(x^{\prime}, t\right)$ is lower semicontinuous in $x^{\prime}$. Equations (5.4) and (5.5) imply that

$$
x_{0} \notin \Omega^{\prime} \text {. }
$$

Now we will investigate possible dispositions of $y_{0}, y\left(x_{0}^{\prime}, t^{\prime}\right)$, and $t^{\prime}$.

(i) If $y_{0}=y\left(x_{0}^{\prime}, t^{\prime}\right)>t^{\prime}$, then $x_{0} \in \partial \Omega^{\prime}$ contradicting the assumption (b). 
(ii) The second possibility is

$$
y_{0}>y\left(x_{0}^{\prime}, t^{\prime}\right) \geq t^{\prime} .
$$

It follows from the assumption (b) and (5.6) that there exists a ball $B_{\varepsilon_{1}}^{(n)}\left(x_{0}\right)$ with a small radius $\varepsilon_{1}>0$ such that

$$
\overline{B_{\varepsilon_{1}}^{(n)}}\left(x_{0}\right) \subset\left(\mathbb{R}^{n} \backslash \Omega^{\prime}\right) \cap H_{+}\left(t^{\prime}\right) .
$$

By (5.4), there exists a point $\hat{x} \in \Omega \cap B_{\varepsilon_{1}}^{(n)}\left(x_{0}\right)$. Since $B_{\varepsilon_{1}}^{(n)}\left(x_{0}\right) \subset H_{+}\left(t^{\prime}\right)$ we must have $\hat{x} \in \Omega^{\prime}$. But this contradicts (5.7) and therefore contradicts (b).

(iii) Let $y_{0}=t^{\prime}$. The assumption (b) and (5.6) imply that there is a ball $B_{\varepsilon_{1}}^{(n)}\left(x_{0}\right)$ such that $B_{\varepsilon_{1}}^{(n)}\left(x_{0}\right) \subset \mathbb{R}^{n} \backslash \Omega^{\prime}$. Then it follows from the definition of $\Omega^{t^{\prime}}$ that $B_{\varepsilon}^{(n)}\left(x_{0}\right) \subset$ $\mathbb{R}^{n} \backslash \Omega$, which contradicts (5.4). This finishes the proof of the lemma in the case (1) under the assumption (b).

(2) In the second case we assume that

$$
x_{k} \in \partial\left(\Omega_{-}^{*}\left(t_{k}\right)\right) \backslash \partial \Omega_{+}\left(t_{k}\right) \quad \text { for all } k=1,2, \ldots
$$

As in the case (1) we consider possible dispositions of $y_{0}, y\left(x_{0}^{\prime}, t^{\prime}\right)$, and $t^{\prime}$.

(i) If $y_{0}<t^{\prime}$, then $y_{k}<t_{k}$ for all sufficiently large $k$. Since $x_{k} \in \partial\left(\Omega_{-}^{*}\left(t_{k}\right)\right)$ and since the open interval between the points $\left(x_{k}^{\prime}, 2 t_{k}-y\left(x_{k}^{\prime}, t_{k}\right)\right)$ and $\left(x_{k}^{\prime}, y\left(x_{k}^{\prime}, t_{k}\right)\right)$ is in $\Omega_{-}^{*}\left(t_{k}\right)$ we have

$$
y_{k} \leq 2 t_{k}-y\left(x_{k}^{\prime}, t_{k}\right) \leq 2 t^{\prime}-y\left(x_{k}^{\prime}, t^{\prime}\right)
$$

for all $k$ large enough. The second inequality here follows from (4.4). The latter inequality implies that $x_{k} \notin \Omega^{\prime}$ and therefore $x_{0}$ is not an inner point of $\Omega^{\prime}$.

Let us show that $x_{0}$ cannot be an outer point of $\Omega^{\prime}$, too. Since $x_{k} \in \partial \Omega_{-}^{*}\left(t_{k}\right)$ and $x_{k} \rightarrow x_{0}$ there is a sequence of points $\hat{x}_{k}=\left(\hat{x}_{k}^{\prime}, \hat{y}_{k}\right) \in \Omega_{-}^{*}\left(t_{k}\right)$ such that $\hat{x}_{k} \rightarrow x_{0}$. Then we have $2 t_{k}-y\left(\hat{x}_{k}^{\prime}, t_{k}\right)<\hat{y}_{k}$. Therefore using (4.4) we obtain

$$
2 t^{\prime}-y\left(\hat{x}_{k}^{\prime}, t^{\prime}\right) \leq 2 t_{k}-y\left(\hat{x}_{k}^{\prime}, t_{k}\right)+\left(t^{\prime}-t_{k}\right)<\left(t^{\prime}-t_{k}\right)+\hat{y}_{k} .
$$

This yields $\left(\hat{x}_{k}^{\prime},\left(t^{\prime}-t_{k}\right)+\hat{y}_{k}\right) \in \Omega^{\prime}$. Since $x_{0} \notin \Omega^{\prime}$ taking the limit we get $x_{0} \in \partial \Omega^{\prime}$, which contradicts the assumption (b).

(ii) Let $y_{0}>t^{\prime}$. Then $y\left(x_{k}^{\prime}, t_{k}\right) \leq y_{k}$ for all $k$ large enough. Therefore by (4.4),

$$
y\left(x_{k}^{\prime}, t^{\prime}\right) \leq 2\left(t^{\prime}-t_{k}\right)+y\left(x_{k}^{\prime}, t_{k}\right) \leq 2\left(t^{\prime}-t_{k}\right)+y_{k} .
$$

Passing to the limit and using the semi-continuity property of the separating function we obtain

$$
y\left(x_{0}^{\prime}, t^{\prime}\right) \leq y_{0} .
$$

The case of equality $y\left(x_{0}^{\prime}, t^{\prime}\right)=y_{0}\left(>t^{\prime}\right)$ leads to the inclusion $x_{0} \in \partial \Omega^{\prime}$, which contradicts the assumption (b). Indeed, if $x_{0} \notin \partial \Omega^{\prime}$, then $x_{0} \in \Omega^{\prime}$. Therefore $x_{k} \in \Omega$ for all sufficiently large $k$. Since $x_{k} \in \partial \Omega_{-}^{*}\left(t_{k}\right)$ and $x_{k} \in \Omega$, it follows that $x_{k} \in \Omega_{+}\left(t_{k}\right)$. Therefore $x_{k} \in \partial \Omega_{+}\left(t_{k}\right)$ for all $k$ large enough, which contradicts (5.8).

Now let $y\left(x_{0}^{\prime}, t^{\prime}\right)<y_{0}$. If $x_{0} \notin \Omega^{\prime}$, then it follows from the assumption (b) that there is a ball $B_{\varepsilon_{2}}^{(n)}\left(x_{0}\right)$ with some small radius $\varepsilon_{2}>0$ such that

$$
\overline{B_{\varepsilon_{2}}^{(n)}}\left(x_{0}\right) \subset\left(\mathbb{R}^{n} \backslash \Omega^{\prime}\right) \cap H_{+}\left(t^{\prime}\right) .
$$


FigURE 1. $T^{t}$-transformation of sets.

Then $\overline{B_{\varepsilon_{2}}^{(n)}}\left(x_{0}\right) \cap \Omega=\emptyset$. Since $\overline{B_{\varepsilon_{2}}^{(n)}}\left(x_{0}\right) \subset H_{+}\left(t_{k}\right)$ we must have $\overline{B_{\varepsilon_{2}}^{(n)}}\left(x_{0}\right) \cap \Omega_{k}=\emptyset$ for all $k=1,2, \ldots$ The latter is impossible since $x_{k} \in \partial \Omega_{k}$ and $x_{k} \rightarrow x_{0}$.

If $x_{0} \in \Omega^{\prime}$ and $y\left(x_{0}^{\prime}, t^{\prime}\right)<y_{0}$, then $x_{0} \in \Omega$. Hence, $x_{k} \in \Omega$ for all sufficiently large $k$. Since $x_{k} \in \partial \Omega_{-}^{*}\left(t_{k}\right)$ this implies that $x_{k} \in \Omega_{k}$ contradicting the assumption (b) if $k$ is large enough. This completes the proof of the lemma in the case $y_{0}>t^{\prime}$.

(iii) Let $y_{0}=t^{\prime}$. If $x_{0} \notin \Omega^{\prime}$ then by the assumption (b), $\overline{B_{\varepsilon}^{(n)}}\left(x_{0}\right) \cap \Omega^{\prime}=\emptyset$. This implies that $\overline{B_{\varepsilon}^{(n)}}\left(x_{0}\right) \cap \Omega_{-}^{*}\left(t_{k}\right)=\emptyset$ for all $k=1,2, \ldots$ The latter contradicts the assumptions $x_{k} \in \partial \Omega_{-}^{*}\left(t_{k}\right)$ and $x_{k} \rightarrow x_{0}$.

Let $x_{0} \in \Omega^{\prime}$. Then by the assumption (b), $B_{\varepsilon}^{(n)}\left(x_{0}\right) \subset \Omega_{-}^{*}\left(t^{\prime}\right)$. This together with the inequality (4.4) implies that $B_{\varepsilon / 2}^{(n)}\left(x_{0}\right) \subset \Omega_{-}^{*}\left(t_{k}\right)$ for all $k$ large enough, again contradicting the assumption (b). The proof of the lemma for open sets is now complete.

Figure 3 shows the results of continuous symmetrization of a domain $\Omega$ for a few values of $t$. Comparing domains in Figure $3 \mathrm{c}$ and Figure $3 \mathrm{~d}$, the reader may see that the $T^{t}$-transformation is not continuous from the right in the Hausdorff metric.

Now we will prove the lemma characterizing a stability property of the boundary $\partial \Omega^{t}$.

Lemma 5.2. Let $-\infty<s<t<\infty$. Then

$$
\partial \Omega^{t} \subset \partial \Omega^{s}+(t-s) \overline{B^{(n)}}
$$

if $\Omega$ is open and

$$
\Omega^{t} \subset \Omega^{s}+(t-s) \overline{B^{(n)}}
$$

if $\Omega$ is a compact.

Proof. Once more we give a proof for open sets. The easier case of compact sets is left to the reader.

Suppose that $\Omega$ is open and that (5.11) is not satisfied. Then there exists a point $x_{0} \in \partial \Omega^{t}$ such that

$$
\operatorname{dist}\left(x_{0}, \partial \Omega^{s}\right)>(t-s) .
$$

By Lemma 4.3(a), we have $\Omega^{t}=\Omega_{-}^{*}(t) \cup \Omega_{+}(t)$. Hence,

$$
\partial \Omega^{t} \subset \partial \Omega_{-}^{*}(t) \cup \partial \Omega_{+}(t) .
$$

As in the proof of Lemma 5.1 we consider two cases.

(1) In the first case we assume that

$$
x_{0} \in \partial \Omega_{+}(t) .
$$

By Lemma 4.3(c), the set $\Omega_{-}(t)$ is open. By the definitions of $\Omega_{-}(t)$ and $\Omega_{+}(t)$, we have $\Omega_{-}(t) \cap \Omega_{+}(t)=\emptyset$. Therefore (5.14) implies that $x_{0} \notin \Omega_{-}(t)$. 
Since $\Omega_{+}(t) \subset \Omega$ we must have $x_{0} \in \bar{\Omega}$. If $x_{0} \in \Omega$, we conclude that $x_{0} \in \Omega_{+}(t)$ and therefore $x_{0} \in \Omega^{t}$. Since by Lemma 4.3(c), $\Omega^{t}$ is open, the latter contradicts the assumption $x_{0} \in \partial \Omega^{t}$. Therefore we must have

$$
x_{0} \in \partial \Omega .
$$

Since $\Omega_{+}(t) \subset \Omega_{+}(s) \subset \Omega$, equations (5.14) and (5.15) imply

$$
x_{0} \in \partial \Omega_{+}(s) .
$$

Hence, $x_{0} \in \overline{\Omega^{s}}$. This implies that $x_{0} \in \partial \Omega^{s}$. Indeed, let $x_{0} \in \Omega^{s}$. Since $x_{0} \notin \Omega$ we conclude that $x_{0} \notin \Omega_{+}(s)$. Therefore in the case under consideration we must have

$$
x_{0} \in \Omega_{-}^{*}(s) .
$$

By Lemma 4.3(c), the set $\Omega_{-}^{*}(s)$ is open and it follows from the definitions that $\Omega_{-}^{*}(s) \cap \Omega_{+}(s)=\emptyset$. Therefore (5.17) contradicts (5.16) and we must have $x_{0} \in \partial \Omega^{s}$ contradicting (5.13).

(2) In the second case we assume that $x_{0}=\left(x_{0}^{\prime}, y_{0}\right) \in \partial \Omega_{-}^{*}(t) \backslash \partial \Omega_{+}(t)$. Now we consider two subcases.

(i) Suppose that $y_{0} \leq t$. Since $x_{0} \in \Omega^{t}$ we have

$$
y_{0} \leq 2 t-y\left(x_{0}^{\prime}, t\right) \text {. }
$$

This together with (4.4) implies

$$
y_{0}-(t-s) \leq 2 s-y\left(x_{0}^{\prime}, s\right)
$$

This inequality yields

$$
\tilde{x}:=\left(x_{0}^{\prime}, y_{0}-(t-s)\right) \notin \Omega^{s} .
$$

Since $x_{0} \in \partial \Omega_{-}^{*}(t)$ then for each $\varepsilon>0$ there exists a point $\hat{x}=\left(\hat{x}^{\prime}, \hat{y}\right) \in \Omega_{-}^{*}(t)$ such that

$$
\left|\hat{x}-x_{0}\right|<\varepsilon .
$$

Let $y_{0}<s$. Then $m\left(\hat{x}^{\prime}, \hat{y}\right)>0$ and therefore $m\left(\hat{x}^{\prime}, s\right)>0$ if $\hat{x}$ is close enough to $x_{0}$. Therefore the interval

$$
\left(\left(\hat{x}^{\prime}, y\right): 2 s-y\left(\hat{x}^{\prime}, s\right)<y<y\left(\hat{x}^{\prime}, s\right)\right)
$$

is nonempty and belongs to $\Omega^{s}$.

Using (4.4) and the relation $\hat{x} \in \Omega_{-}^{*}(t)$ we conclude that

$$
2 s-y\left(\hat{x}^{\prime}, s\right) \leq 2 t-y\left(\hat{x}^{\prime}, t\right)<\hat{y}<s
$$

if $\varepsilon>0$ in (5.19) is small enough. Then we have $\hat{x} \in \Omega^{s}$. Therefore the closed interval $[\hat{x}, \tilde{x}]$ contains a point $z \in \partial \Omega^{s}$. Hence,

$$
\left|z-x_{0}\right| \leq \max \left\{\left|x_{0}-\tilde{x}\right|,\left|x_{0}-\hat{x}\right|\right\}=(t-s)
$$

if $\varepsilon>0$ in (5.19) is $\leq t-s$, which contradicts inequality (5.13).

Let $y_{0}=s$ and let $\hat{y} \leq s$. Then $m\left(\hat{x}^{\prime}, s\right)>0$ and $\left(\hat{x}^{\prime}, s\right) \in \Omega^{s}$. The closed interval $\left[x_{0},\left(\hat{x}^{\prime}, s\right)\right]$ contains a point $z \in \partial \Omega^{s}$. Hence, $\left|x_{0}-z\right| \leq \varepsilon$, which again contradicts (5.13) if $\varepsilon<t-s$.

Let $y_{0}=s, \hat{y}>s$. If $m\left(\hat{x}^{\prime}, s\right)>0$, then we argue as in the previous case. 
Let $m\left(\hat{x}^{\prime}, s\right)=0$. Since $m\left(\hat{x}^{\prime}, \hat{y}\right)>0$ then there exists a point $z=\left(\hat{x}^{\prime}, \zeta\right) \in \Omega$ such that $s<\zeta \leq \hat{y}$. But in this case $z \in \Omega^{s}$ and $\left|z-x_{0}\right| \leq\left|\hat{x}-x_{0}\right| \leq \varepsilon$. Since $\varepsilon>0$ is arbitrary small this easily leads to a contradiction to (5.13).

Let $s<y_{0} \leq t$. If $m\left(\hat{x}^{\prime}, s\right)>0$, then $\left(\hat{x}^{\prime}, s\right) \in \Omega^{s}$ and

$$
\left|x_{0}-\left(\hat{x}^{\prime}, s\right)\right| \leq\left|x_{0}-\left(x_{0}^{\prime}, s\right)\right|+\left|\left(x_{0}^{\prime}, s\right)-\left(\hat{x}^{\prime}, s\right)\right| \leq(t-s)+\varepsilon .
$$

Since $\varepsilon>0$ can be chosen arbitrary small the latter inequality contradicts (5.13).

Let $m\left(\hat{x}^{\prime}, s\right)=0$. Since $m\left(\hat{x}^{\prime}, \hat{y}\right)>0$ then there exists a point $z=\left(\hat{x}^{\prime}, \zeta\right) \in \Omega$ such that $s<\zeta<\hat{y}$. Then $z \in \Omega^{s}$. In addition,

$$
\left|z-x_{0}\right| \leq(t-s)+\varepsilon
$$

Since $\varepsilon>0$ can be chosen arbitrary small the latter inequality again contradicts (5.13).

(ii) Let $y_{0}>t$. Since $x_{0} \notin \Omega^{t}$ we have $y_{0} \geq y\left(x_{0}^{\prime}, t\right) \geq y\left(x_{0}^{\prime}, s\right)$. This implies that $x_{0} \notin \Omega^{s}$. Since $x_{0} \in \partial \Omega_{-}^{*}(t)$ we conclude that for every $\varepsilon>0$ there exists a point $\hat{x}=\left(\hat{x}^{\prime}, \hat{y}\right) \in \Omega_{-}^{*}(t)$ such that

$$
\left|\hat{x}-x_{0}\right|<\varepsilon .
$$

If $\varepsilon>0$ is small enough we have $t<\hat{y}<y\left(\hat{x}^{\prime}, t\right)$.

If $y\left(\hat{x}^{\prime}, s\right) \geq \hat{y}$, then $\hat{x} \in \overline{\Omega^{s}}$. Therefore (5.20) contradicts (5.13).

Now we consider the case $y\left(\hat{x}^{\prime}, s\right)<\hat{y}$. Let $I:=\left(\left(\hat{x^{\prime}}, y\left(\hat{x}^{\prime}, t\right)\right),\left(\hat{x}^{\prime}, y\left(\hat{x}^{\prime}, s\right)\right)\right.$ and let $\Omega_{I}=I \cap \Omega$. It follows from (4.3) that the linear measure of $\Omega_{I}$ equals $2\left(y\left(\hat{x}^{\prime}, t\right)-\right.$ $\left.y\left(\hat{x^{\prime}}, s\right)\right)-2(t-s)$. Since $\hat{x} \in I$ we have

$$
\operatorname{dist}\left(\hat{x}, \Omega_{I}\right) \leq \mathcal{L}^{1}(I)-\mathcal{L}^{1}\left(\Omega_{I}\right) .
$$

This inequality together with (4.4) implies

$$
\operatorname{dist}\left(\hat{x}, \Omega_{I}\right) \leq 2(t-s)-\left(y\left(\hat{x^{\prime}}, t\right)-y\left(\hat{x^{\prime}}, s\right)\right) \leq t-s .
$$

Since $\Omega_{I} \subset \Omega^{s}$ there exists a point $z \in \overline{\Omega^{s}}$ such that $|\hat{x}-z| \leq t-s$. Finally, we have

$$
\left|x_{0}-z\right| \leq\left|x_{0}-\hat{x}\right|+|\hat{x}-z| \leq(t-s)+\varepsilon \text {. }
$$

Since $\varepsilon>0$ can be chosen arbitrary small the latter inequality contradicts (5.13). The proof of the lemma for open sets is complete.

\section{Stability And Semigroup PROPERTIEs of $T^{t}$-TRANSFORMATion}

First we prove a lemma characterizing stability with respect to polarization, cf. [27, Theorem 3].

Lemma 6.1. For $s \in \mathbb{R}$, let $P_{s}$ denote the polarization with the polarizer $H_{s}=$ $\left\{\left(x^{\prime}, y\right) \in \mathbb{R}^{n}: y>s\right\}$. If $t_{1}<t_{2}$ and $s \leq(1 / 2)\left(t_{1}+t_{2}\right)$, then

$$
T^{t_{2}} \circ P_{s} \circ T^{t_{1}}=T^{t_{2}} \quad \text { on } \mathcal{M}_{n} \text {. }
$$

Proof. Let $\Omega \in \mathcal{M}$ be an open set and let $D=P_{s}\left(\Omega^{t_{1}}\right)$. We have to show that

$$
D^{t_{2}}=\Omega^{t_{2}} \text {. }
$$

By Lemma 4.3(a),

$$
D^{t_{2}}=D_{-}^{*}\left(t_{2}\right) \cup D_{+}\left(t_{2}\right), \quad \Omega^{t_{2}}=\Omega_{-}^{*}\left(t_{2}\right) \cup \Omega_{+}\left(t_{2}\right) .
$$


Since the $T^{t}$-transformation and polarization both preserve measure in 1-slices, it follows from (6.3) that (6.2) holds true if and only if

$$
D_{+}\left(t_{2}\right)=\Omega_{+}\left(t_{2}\right) .
$$

Since $\Omega^{t_{1}} \cap\left\{\left(x^{\prime}, y\right): y<2 t_{1}-y_{\Omega}\left(x^{\prime}, t_{1}\right)\right\}=\emptyset$, it follows from the definition of polarization and $T^{t}$-transformation that

$$
\begin{aligned}
P_{s} & \left(\Omega^{t_{1}}\right) \cap\left\{\left(x^{\prime}, y\right): y \geq 2\left(s-t_{1}\right)+y_{\Omega}\left(x^{\prime}, t_{1}\right)\right\} \\
& =\Omega^{t_{1}} \cap\left\{\left(x^{\prime}, y\right): y \geq 2\left(s-t_{1}\right)+y_{\Omega}\left(x^{\prime}, t_{1}\right)\right\} \\
& =\Omega \cap\left\{\left(x^{\prime}, y\right): y \geq 2\left(s-t_{1}\right)+y_{\Omega}\left(x^{\prime}, t_{1}\right)\right\} .
\end{aligned}
$$

Since $s \leq(1 / 2)\left(t_{1}+t_{2}\right),(4.4)$ implies

$$
y_{\Omega}\left(x^{\prime}, t_{2}\right) \geq y_{\Omega}\left(x^{\prime}, t_{1}\right)+2\left(s-t_{1}\right) .
$$

Now from (6.5) and (6.6) we conclude that

$$
P_{s}\left(\Omega^{t_{1}}\right) \cap\left\{\left(x^{\prime}, y\right): y \geq y_{\Omega}\left(x^{\prime}, t_{2}\right)\right\}=\Omega \cap\left\{\left(x^{\prime}, y\right): y \geq y_{\Omega}\left(x^{\prime}, t_{2}\right)\right\},
$$

which implies (6.4).

For open sets the proof is complete. If $\Omega$ is not open the proof follows the same lines.

Lemma 6.1 and Definition 4.4 immediately imply:

Corollary 6.1. Let $u(x) \in \mathcal{S}_{n}, t_{1}<t_{2}$, and let $s \leq(1 / 2)\left(t_{1}+t_{2}\right)$. Then

$$
\left(\left(u^{t_{1}}\right)_{H_{s}}\right)^{t_{2}}=u^{t_{2}}
$$

In the particular case $s=t_{1}$, Lemma 6.1 and its corollary show that our $T^{t}$-transformation possesses the following "presemigroup property", which was first mentioned in [27]. A similar property was used in [13] to define the BC symmetrization.

Corollary 6.2. Let $T^{t}$ denote a $T^{t}$-transformation on $\mathcal{M}_{n}$ or on $\mathcal{S}_{n}$. Then

$$
T^{t_{2}} \circ T^{t_{1}}=T^{t_{2}} \quad \text { for all } t_{1}<t_{2} .
$$

To justify the name "presemigroup property", we consider the transformation $\widehat{T}^{t}=$ $S t^{t} \circ T^{t}$, where $t \geq 0$ and $S t^{t}$ denotes a continuous shift of $t$ units in the direction of the negative $y$-axis. Then one can easily see that equation (6.7) is equivalent to the following familia semigroup property of $\widehat{T}^{t}$ :

$$
\widehat{T}^{t+s}=\widehat{T}^{t} \circ \widehat{T}^{s} \quad \text { for all } t \geq 0, s \geq 0 .
$$

In Section 8, a similar composite transformation will be used to replace the parameter $t$, the range of which is $-\infty<t<\infty$, by a parameter $\tau$ with the standard range $0 \leq \tau \leq 1$.

Now we prove a criterion for $\Omega$ and $u$ to possess a partial symmetry .

Lemma 6.2. (a) If $\Omega \in \mathcal{M}_{n}$ is open or compact, then $\Omega=\Omega^{t}$ if and only if $P_{s}(\Omega)=\Omega$ for all $s \leq t$.

(b) If $u \in \mathcal{S}_{n}$, then $u \equiv u^{t}$ if and only if $u_{H_{s}} \equiv u$ for all $s \leq t$. 
Proof. (a) We work with an open set $\Omega$. The necessary part is obvious: If $\Omega=\Omega^{t}$, then Lemma 4.3(a) shows that $P_{s}(\Omega)=\Omega$ for all $s \leq t$.

To prove the sufficient part, we assume that $P_{s}(\Omega)=\Omega$ for all $s \leq t$ but $\Omega \neq \Omega^{t}$. Since $\Omega_{+}(t)=\left(\Omega^{t}\right)_{+}(t)$ we conclude that $\Omega_{-}(t) \neq \Omega_{-}^{*}(t)$. Then by Lemma 6.3 in [14], there is a polarizer $H_{s_{0}}=\left\{y>s_{0}\right\}$ with $s_{0}<t$ such that

$$
P_{s_{0}}\left(\Omega_{-}(t)\right) \neq \Omega_{-}(t) .
$$

Then there is $x_{0}=\left(x_{0}^{\prime}, y_{0}\right)$ with $y_{0}<s_{0}$ such that

$$
x_{0} \in \Omega_{-}(t) \quad \text { and } \quad \hat{x}:=\left(x_{0}^{\prime}, 2 s_{0}-y_{0}\right) \notin \Omega_{-}(t) .
$$

If $2 s_{0}-y_{0}<y\left(x_{0}^{\prime}, t\right)$, then $(6.9)$ contradicts the assumption $P_{s}(\Omega)=\Omega$ for $s=s_{0}<t$. Let $2 s_{0}-y_{0} \geq y\left(x_{0}^{\prime}, t\right)$. Since $P_{s}(\Omega)=\Omega$ for all $s \leq t$ and $x_{0} \in \Omega$, it follows that the closed interval $\left\{\left(x_{0}^{\prime}, y\right): y_{0} \leq y \leq 2 t-s_{0}\right\}$ is in $\Omega$. Then

$$
m_{\Omega}\left(x_{0}^{\prime}, y\left(x_{0}^{\prime}, t\right)\right)>y\left(x_{0}^{\prime}, t\right)-y_{0} \geq 2\left(y\left(x_{0}^{\prime}, t\right)-s_{0}\right) \geq 2\left(y\left(x_{0}^{\prime}, t\right)-t\right)
$$

contradicting the equation (4.3). The proof of Lemma 6.2(a) for open sets is complete. If $\Omega$ is not open the proof is left to the reader.

The proof of Lemma 6.2(b) easily follows from Lemma 6.2(a) and Definition 4.4.

Our next result will be used in the proof of Theorem 11.3 in Section 11.

Corollary 6.3. Let $\Omega$ be a bounded domain in $\mathbb{R}^{n}$ and let $f \in L_{+}^{2}(\Omega), f>0$ on $\Omega$. If for some $T \in \mathbb{R}, f^{T}$ is not a translation of $f$ in the direction of $y$-axis, then we can find a polarizer $H_{t_{0}}=\left\{y>t_{0}\right\}$ with $t_{0}<T$ such that $\left(f_{H_{t_{0}}}\right)^{T}=f^{T}, f \neq f_{H_{t_{0}}}$, and $\sigma_{H_{t_{0}}}(f) \neq f_{H_{t_{0}}}$.

In particular, if $\Omega^{T}$ is not a translation of $\Omega$ in the direction of $y$-axis, then we can find a polarizer $H_{t_{0}}=\left\{y>t_{0}\right\}$ with $t_{0}<T$ such that $\left(\Omega_{H_{t_{0}}}\right)^{T}=\Omega^{T}, \Omega \neq \Omega_{H_{t_{0}}}$, and $\sigma_{H_{t_{0}}}(\Omega) \neq \Omega_{H_{t_{0}}}$.

Proof. Let $t_{1}=\inf t$, where the infimum is taken over all $t \in \mathbb{R}$ such that $\Omega_{+}(t)=\emptyset$. Then for all $t \geq t_{1}, f^{t}$ is a translation of $f^{t_{1}}$. Therefore, proving this corollary, we may assume that $T \leq t_{1}$. In this case, $f^{T}$ is not a translation of $f$ if and only if $f^{T} \neq f$.

Let $t_{\Omega}=\sup t$, where the supremum is taken over all $t \in \mathbb{R}$ such that $P_{\tau}(f)=f$ for all $\tau \leq t$. Then, $f^{t_{\Omega}}=f$ by Lemma 6.2. Since $f^{T} \neq f$, it follows from the same lemma that $t_{\Omega}<T$. Combining this with Corollary 6.1 , we obtain that

$$
\left(f_{H_{s}}\right)^{T}=\left(\left(f^{t_{\Omega}}\right)_{H_{s}}\right)^{T}=f^{T} \text { for all } s \leq(1 / 2)\left(t_{\Omega}+T\right) .
$$

Since $t_{\Omega}<T, f^{t_{\Omega}}=f$, and $\Omega_{+}\left(t_{\Omega}\right) \neq \emptyset$, we must have $\sigma_{H_{s}}(\Omega) \neq \Omega_{H_{s}}$, and therefore $\sigma_{H_{s}}(f) \neq f_{H_{s}}$, for all $s$ sufficiently close to $t_{\Omega}$. Therefore, every polarizer $H_{s}$ with $t_{\Omega}<s<(1 / 2)\left(t_{\Omega}+T\right)$ such that $f_{H_{s}} \neq f$ will satisfy the requirements of the corollary.

By the definition of $t_{\Omega}$, there is a sequence $\left\{s_{k}\right\}_{k=1}^{\infty}$, which converges to $t_{\Omega}$ from the right, such that $f_{H_{s_{k}}} \neq f$ for all $k$. Choosing $k$ sufficiently large such that $s_{k} \leq$ $(1 / 2)\left(t_{\Omega}+T\right)$, we obtain the desired polarizer $H_{t_{0}}=H_{s_{k}}$. This proves the first assertion of the corollary. Since $\Omega=\{f>0\}$ the second assertion is a particular case of the first one.

Lemma 6.3. Let $\Omega \in \mathcal{M}_{n}$ and let $(\cdot)^{*}$ denote the $(1, n)$-Steiner symmetrization with respect to the plane $\{y=t\}$. If $T^{t}(\Omega)=\Omega^{*}$ and $\Omega \in \mathcal{F} \cup \mathcal{G}_{b}$ then $T^{t}\left(\Omega_{\varepsilon}\right)=\left(\Omega_{\varepsilon}\right)^{*}$ for every $\varepsilon>0$. 
Proof. Assume that $\Omega \in \mathcal{G}_{b}$. For a fixed $\varepsilon>0$, let $y_{\varepsilon}\left(x^{\prime}, t\right)$ and $m_{\varepsilon}\left(x^{\prime}, y\right)$ denote, respectively, the separating function and measuring function of $\Omega_{\varepsilon}$. For $x^{\prime} \in\left(\Omega_{\varepsilon}\right)^{\prime}$, let

$$
y_{\varepsilon}\left(x^{\prime}\right)=\sup \left\{y:\left(x^{\prime}, y\right) \in T^{t}\left(\Omega_{\varepsilon}\right)\right\} .
$$

Then $y_{\varepsilon}\left(x^{\prime}\right) \geq y_{\varepsilon}\left(x^{\prime}, t\right)$. If $y_{\varepsilon}\left(x^{\prime}\right)=y_{\varepsilon}\left(x^{\prime}, t\right)$ for all $x^{\prime} \in\left(\Omega_{\varepsilon}\right)^{\prime}$, the conclusion of the lemma is immediate.

Suppose that

$$
y_{\varepsilon}\left(x_{0}^{\prime}\right)>y_{\varepsilon}\left(x_{0}^{\prime}, t\right)
$$

for some $x_{0}^{\prime} \in\left(\Omega_{\varepsilon}\right)^{\prime}$. Then (4.3) implies

$$
m_{\varepsilon}\left(x_{0}^{\prime}, y_{\varepsilon}\left(x_{0}^{\prime}\right)\right)<2\left(y_{\varepsilon}\left(x_{0}^{\prime}\right)-t\right) .
$$

Now we fix $\delta>0$ sufficiently small. Then we can find two points $\hat{x}=\left(x_{0}^{\prime}, \hat{y}\right)$ and $\tilde{x}=\left(\tilde{x}^{\prime}, \tilde{y}\right)$ satisfying the following conditions:

$$
\begin{gathered}
\hat{x} \in T^{t}\left(\Omega_{\varepsilon}\right) \quad \text { and } \quad y_{\varepsilon}\left(x_{0}^{\prime}\right)-\delta<\hat{y}<y_{\varepsilon}\left(x_{0}^{\prime}\right), \\
\tilde{x} \in \Omega \quad \text { and } \quad|\hat{x}-\tilde{x}|<\varepsilon .
\end{gathered}
$$

Since $T^{t}(\Omega)=\Omega^{*}$ and $\tilde{x} \in \Omega$ we have:

$$
m\left(\tilde{x}^{\prime}, \tilde{y}\right) \geq 2(\tilde{y}-t) .
$$

Using (6.12) and (6.13), after an elementary geometric argument we obtain the inequality

$$
m_{\varepsilon}\left(x_{0}^{\prime}, \hat{y}\right) \geq 2(\hat{y}-t),
$$

which together with (6.11) implies

$$
m_{\varepsilon}\left(x_{0}^{\prime}, y_{\varepsilon}\left(x_{0}^{\prime}\right)\right) \geq m_{\varepsilon}\left(x_{0}^{\prime}, \hat{y}\right) \geq 2\left(y_{\varepsilon}\left(x_{0}^{\prime}\right)-t\right)-2 \delta .
$$

Since $\delta>0$ can be chosen arbitrary small, the latter inequality contradicts (6.10). This proves the lemma for open sets. If $\Omega$ is a compact set the proof follows the same lines.

\section{Approximation by polarizations}

A compact set $K \subset \mathbb{R}^{n}$ is said to be simple if it can be decomposed into a finite number of blocks $\bar{R}_{i}=\bar{R}\left(a^{i}, b^{i}\right)$ such that $R_{i} \cap R_{j}=\emptyset$ if $i \neq j$. Here $\bar{R}(a, b)$ denotes the closure of an open block

$$
R(a, b):=\left\{x \in \mathbb{R}^{n}: b_{k}<x_{k}<b_{k}+a_{k}, 1 \leq k \leq n\right\},
$$

where $a=\left(a_{1}, \ldots, a_{n}\right) \in \mathbb{R}_{+}^{n}$ and $b=\left(b_{1}, \ldots, b_{n}\right) \in \mathbb{R}^{n}$.

If $K$ is a simple compact set in $\mathbb{R}^{n}=\mathbb{R}^{n-1} \times \mathbb{R}, n \geq 2$, then its projection $K^{\prime}$ is a simple compact set in $\mathbb{R}^{n-1}$. Let $K^{\prime}=\cup_{i=1}^{N} \bar{R}_{i}^{\prime}$ be a block decomposition of $K^{\prime}$.

A collection of open sets $C u(K):=\left\{C_{i}=R_{k}^{\prime} \times \mathbb{R}\right\}_{1}^{N}$ will be called a cubicle structure of $K$. If $C_{i}$ is a cubicle in $C u(K)$, then $C_{i} \cap K=R_{i}^{\prime} \times K^{i}$, where $K^{i}$ is a simple compact set in $\mathbb{R}$. Therefore $C_{i} \cap K$ consists of a finite number of disjoint blocks $K_{i, j}=R_{i}^{\prime} \times\left[\alpha_{i j}, \beta_{i j}\right], 1 \leq j \leq j_{i}$. We always enumerate these blocks such that

$$
\alpha_{i 1}<\beta_{i 1}<\alpha_{i 2}<\cdots<\alpha_{i j_{i}}<\beta_{i j_{i}}, \quad 1 \leq i \leq N .
$$

Thus every simple compact set $K$ admits a block decomposition of the form

$$
K=\bigcup_{i=1}^{N} \bar{R}_{i}^{\prime} \times K^{i}=\bigcup_{i, j} \bar{K}_{i j} .
$$


In this section we show that the $T^{t}$-transformation of sets and functions can be approximated by a sequences of polarizations. This approach was first used by V. Wolontis [33]. The method was developed further by V. N. Dubinin [16], [17]. So we will call this method the Wolontis-Dubinin approach. In the context of continuous symmetrization the Wolontis-Dubinin approach was first used in [27].

Lemma 7.1. For every $t \in \mathbb{R}$ and every collection of simple compact sets $K_{1}, \ldots, K_{m}$ in $\mathbb{R}^{n}$ there exist a finite number of polarizations $P_{k}, 1 \leq k \leq N$ with polarizers $\left\{y>y_{k}\right\}$ such that

$$
y_{1}<y_{2}<\ldots<y_{N}<t
$$

and

$$
\bigcirc_{k=1}^{N} P_{k}\left(K_{i}\right)=K_{i}^{t} \quad \text { for all } 1 \leq i \leq m .
$$

Proof. (1) Let $t \in \mathbb{R}$ be fixed. First we consider the case $n=1$. Then the block decomposition of $K_{i}$ consists of a finite number of disjoint segments:

$$
K_{i}=\bigcup_{j=1}^{j_{i}}\left[a_{i j}, b_{i j}\right] \text {, }
$$

where

$$
a_{i 1}<b_{i 1}<a_{i 2}<b_{i 2}<\cdots<a_{i j_{i}}<b_{i j_{i}}, \quad j_{i} \geq 1, \quad 1 \leq i \leq m .
$$

For every index $i$ we define two numbers:

$$
\alpha_{i}=(1 / 2)\left(t+(1 / 2)\left(a_{i 1}+b_{i 1}\right)\right),
$$

and

$$
\beta_{i}= \begin{cases}(1 / 2)\left(a_{i 1}+a_{i 2}\right) & \text { if } j_{i}>1 \\ t & \text { otherwise. }\end{cases}
$$

Let $\mathcal{A}_{t}^{(0)}$ denote the set of all numbers $\alpha_{i}$ and all numbers $\beta_{i}$ that are less than $t$. It is easy to verify that $T^{t}\left(\left(K_{i}\right)\right)=K_{i}$ for some $i$ if and only if $\alpha_{i} \geq t$. Therefore if $\mathcal{A}_{t}^{(0)}=\emptyset$, then $\alpha_{i} \geq t$ for all $i$ and there is nothing to prove.

If $\mathcal{A}_{t}^{(0)} \neq \emptyset$, then we set

$$
y_{1}:=\min \left\{\gamma: \gamma \in \mathcal{A}_{t}^{(0)}\right\} .
$$

Now we consider a polarization $P_{1}$ with the polarizer $\left\{y>y_{1}\right\}$. Let $K_{i}^{(1)}=P_{1}\left(K_{i}\right)$, $1 \leq i \leq m$. For each $i$, the compact set $K_{i}^{(1)}$ satisfies the following conditions:

(a) $K_{i}^{(1)}$ is a simple compact set whose block decomposition $K_{i}^{(1)}=$ $\bigcup_{j=1}^{j_{i}^{(1)}}\left[a_{i j}^{(1)}, b_{i j}^{(1)}\right]$ consists of $j_{i}^{(1)}$ disjoint segments, where $j_{i}^{(1)} \leq j_{i}$.

(b) $\left(K_{i}^{(1)}\right)_{+}(t)=\left(K_{i}\right)_{+}(t)$ and therefore $T^{t}\left(K_{i}^{(1)}\right)=T^{t}\left(K_{i}\right)$ for all $1 \leq i \leq m$.

Now starting with simple compact sets $K_{i}^{(1)}, 1 \leq i \leq m$, we define a set $\mathcal{A}_{t}^{(1)}$ of elements $\alpha_{i}^{(1)}<t$ and $\beta_{i}^{(1)}<t$ in the same way as $\mathcal{A}_{t}^{(0)}$ was defined for $K_{i}$, see formulas (7.3)(7.6). As we noticed above, if $\mathcal{A}_{t}^{(1)}=\emptyset$, then $K_{i}^{(1)}=T^{t}\left(K_{i}^{(1)}\right)=T^{t}\left(K_{i}\right), 1 \leq i \leq m$, where the second equality follows from the condition (b). In this case our construction is finished. 
If $\mathcal{A}_{t}^{(1)} \neq \emptyset$, we continue our construction further to get simple compact sets $K_{i}^{(2)}$, $K_{i}^{(3)}, \ldots, 1 \leq i \leq m$. Let $\mathcal{A}_{t}^{(k)}, \alpha_{i}^{(k)}, \beta_{i}^{(k)}$, and $y_{k+1}$ correspond $K_{i}^{(k)}$ in the process of this construction. One can easily see that in each step of our construction $K_{i}^{(k)}, \mathcal{A}_{t}^{(k)}$, and $y_{k+1}$ satisfy the following conditions:

(i) If for some $k \geq 1, \mathcal{A}_{t}^{(k)} \neq \emptyset$, then $y_{1}<y_{2}<\cdots<y_{k}<t$.

(ii) If for some $k \geq 1$ and some $s, 1 \leq s \leq m, y_{k}=\alpha_{s}^{(k-1)}$, then $\# \mathcal{A}_{t}^{(k)} \leq$ $\# \mathcal{A}_{t}^{(k-1)}-1$, where \# denotes the cardinality of the corresponding set and $\mathcal{A}_{t}^{(0)}=\mathcal{A}_{t}$.

(iii) If $y_{k}=\beta_{s}^{(k-1)}$ for some $s, 1 \leq s \leq m$, then $j_{s}^{(k)} \leq j_{s}^{(k-1)}-1$, where $j_{i}^{(l)}$ denotes the number of disjoint segments in $K_{i}^{(l)}$ if $l \geq 1$ and $j_{i}^{(0)}=j_{i}$.

Since the total number of segments constituting $K_{1}, \ldots, K_{m}$ is finite and $\# \mathcal{A}_{t}<\infty$ the conditions (ii) and (iii) show that the above construction of compact sets $K_{i}^{(k)}$ terminates after a finite number of steps. Thus there is an integer $N \geq 0$ such that $\mathcal{A}_{t}^{(N)}=\emptyset$. Since in each step of our construction the conditions (a) and (b) are satisfied we have

$$
K_{i}^{(N)}=T^{t}\left(K_{i}^{(N)}\right)=T^{t}\left(K_{i}^{(N-1)}\right)=\cdots=T^{t}\left(K_{i}\right), \quad 1 \leq i \leq m,
$$

which proves the lemma in the case under consideration.

(2) Now we consider the case $n \geq 2$. Since $K_{i}$ is a simple compact set in $\mathbb{R}^{n}$ it can be decomposed as follows

$$
K_{i}=\bigcup_{j=1}^{j_{i}}{\overline{R^{\prime}}}_{i j} \times K_{i}^{j}
$$

where $\bar{R}_{i j}$ are closed blocks in $\mathbb{R}^{n-1}$ such that $R_{i j}^{\prime} \cap R_{i l}^{\prime}=\emptyset$ if $j \neq l$ and $K_{i}^{j}$ are simple compact sets in $\mathbb{R}$.

By part (1) of this proof, for every $t \in \mathbb{R}$ there are polarizations $P_{k}, 1 \leq k \leq N$, in $\mathbb{R}$ with the polarizers $\left\{y>y_{k}\right\}$ such that

$$
y_{1}<y_{2}<\cdots<y_{N}<t
$$

and

$$
\bigcirc_{k=1}^{N} P_{k}\left(K_{i}^{j}\right)=T^{t}\left(K_{i}^{j}\right) \quad \text { for all } 1 \leq j \leq j_{i} \text { and } 1 \leq i \leq m .
$$

Considering $P_{k}, 1 \leq k \leq N$, as polarizations in $\mathbb{R}^{n}$ with the corresponding polarizers $\left\{y>y_{k}\right\}$ we obtain

$$
\bigcirc_{k=1}^{N} P_{k}\left(K_{i}\right)=\bigcup_{j=1}^{j_{i}} \bar{R}_{i j}^{\prime} \times\left(T^{t}\left(K_{i}^{j}\right)\right)=T^{t}\left(K_{i}\right), \quad 1 \leq i \leq m .
$$

The proof of the lemma is complete.

Corollary 7.1. Let $t \in \mathbb{R}$ and let $D, \Omega$ be bounded open sets such that $\bar{D} \subset \Omega$. Then there is a finite number of polarizations $P_{k}, 1 \leq k \leq N$, with polarizers $\left\{y>y_{k}\right\}$, where $y_{1}<y_{2}<\ldots<y_{N} \leq t$, such that

$$
\bar{D}_{N} \subset \Omega^{t}, \quad \text { where } \bar{D}_{N}=\bigcirc_{k=1}^{N} P_{k}(\bar{D}) \text {. }
$$


In addition,

$$
\operatorname{dist}(\partial D, \partial \Omega) \leq \operatorname{dist}\left(\partial D_{N}, \partial \Omega^{t}\right), \quad \text { where } D_{N}=\bigcirc_{k=1}^{N} P_{k}(D) .
$$

Proof. Let $\rho_{0}=\operatorname{dist}(\partial D, \partial \Omega)$. Then $\rho_{0}>0$ and for every $\rho, 0 \leq \rho<\rho_{0}$, there exists a simple compact set $K=K(\rho)$ such that

$$
(\bar{D})_{\rho} \subset K \subset \Omega \text {. }
$$

By Lemma 7.1, we can find a finite number of polarizations $P_{k}, 1 \leq k \leq N$ such that

$$
K^{t}=K_{N}:=\bigcirc_{k=1}^{N} P_{k}(K) \text {. }
$$

Since the $T^{t}$-transformation and polarization both are monotone and smoothing transformations we have

$$
\Omega^{t} \supset K^{t}=K_{N} \supset \bigcirc_{k=1}^{N} P_{k}\left((\bar{D})_{\rho}\right) \supset\left(\bar{D}_{N}\right) \rho .
$$

Since (7.7) holds true for every $\rho, 0 \leq \rho<\rho_{0}$, the corollary follows.

Definition 7.1. A function $f \in \mathcal{S}_{n}$ is said to be simple if $f$ can be represented as

$$
f=\varepsilon\left(-n_{0}+\sum_{i=1}^{m} \chi\left(K_{i}\right)\right)
$$

with some $\varepsilon>0, n_{0} \in \mathbb{N}$ and some simple compact sets $K_{i}, 1 \leq i \leq m$, such that $K_{1} \supset \cdots \supset K_{m}$.

We note that simple functions are dense in $L_{+}^{p}\left(\mathbb{R}^{n}\right)$ for every $1 \leq p<\infty$. The following lemma shows that the $T^{t}$-transformation of a simple function can be reduced to a finite sequence of polarizations.

Lemma 7.2. Let $f: \mathbb{R}^{n} \rightarrow \mathbb{R}$ be a simple function. Then for every $t \in \mathbb{R}$ there are polarizations $P_{k}$ with polarizers $\left\{y>y_{k}\right\}, 1 \leq k \leq N$ such that

$$
y_{1}<y_{2}<\ldots<y_{N}<t
$$

and

$$
\bigcirc_{k=1}^{N} P_{k}(f)=f^{t}
$$

Proof. Since $f$ is simple it can be represented in the form (7.8). By Lemma 7.1, we can find polarizations $P_{k}, 1 \leq k \leq N$, with the polarizers $\left\{y>y_{k}\right\}$ satisfying (7.9) such that

$$
\bigcirc_{k=1}^{N} P_{k}\left(K_{i}\right)=K_{i}^{t}, \quad 1 \leq i \leq m .
$$

Now (7.10) follows from the relations

$$
\bigcirc_{k=1}^{N} P_{k}(f)=\varepsilon\left(-n_{0}+\sum_{i=1}^{m} \chi\left(\bigcirc_{k=1}^{N} P_{k}\left(K_{i}\right)\right)\right)
$$

and

$$
f^{t}=\varepsilon\left(-n_{0}+\sum_{i=1}^{m} \chi\left(K_{i}^{t}\right)\right),
$$

which in their turn follow from Definition 4.4 and Definition 3.2.

The following lemma shows that, for functions $u \in L_{+}^{p}\left(\mathbb{R}^{n}\right)$ such that $u \neq u^{t}$, one polarization is enough to find a better approximation of $u^{t}$. Its proof, left to the reader, follows the ideas of [7, p.252] and repeats the proof of Lemma 6.4 in [14]. 
Lemma 7.3. Let $u \in L_{+}^{p}\left(\mathbb{R}^{n}\right), 1 \leq p<\infty$. If $u=u^{t_{1}}$ and $u \neq u^{t}$ for some $t_{1}, t \in \mathbb{R}$ such that $t_{1}<t$, then there is a polarizer $H_{s}=\{y>s\}$ with $t_{1}<s<t$ such that

$$
\left(u_{H_{s}}\right)^{t}=u^{t}
$$

and

$$
\left\|u_{H_{s}}-u^{t}\right\|_{p}<\left\|u-u^{t}\right\|_{p}
$$

Lemma 7.4. Let $u \in L_{+}^{p}\left(\mathbb{R}^{n}\right), 1 \leq p<\infty$. Then for every $t \in \mathbb{R}$ there is a sequence of polarizations $P_{k}$ with polarizers $\left\{y>y_{k}\right\}$, where $y_{k} \leq t$ for all $k=1,2, \ldots$, such that the sequence of functions $u_{m}:=\bigcirc_{i=1}^{m} P_{i} u$ satisfies the minimality condition

$$
\left\|u_{m+1}-u^{t}\right\|_{p}=\min \left\{\left\|\left(u_{m}\right)_{H}-u^{t}\right\|_{p}\right\}, \quad m=1,2, \ldots, u_{0}=u,
$$

where the minimum is taken over all polarizations with polarizers $H=\{y>s\}$ such that $-\infty<s \leq t$.

In addition,

$$
u_{m} \rightarrow u^{t} \quad \text { in } L^{p}\left(\mathbb{R}^{n}\right)
$$

Proof. It follows from Lemma 5.2 [14] that the minimum in (7.11) is attained for some polarizer $\left\{y>y_{k+1}\right\},-\infty<y_{k+1} \leq t$. Then by Lemma 6.1 [14], there are some function $v \in L_{+}^{p}\left(\mathbb{R}^{n}\right)$ and a subsequence $u_{m^{\prime}}$ such that

$$
u_{m^{\prime}} \rightarrow v \quad \text { in } L^{p}\left(\mathbb{R}^{n}\right) .
$$

Then using the non-expansivity Lemma 3.1 we obtain $v^{t}=u^{t}$.

Now assume that $v \neq u^{t}$. By Lemma 7.3, we can choose a polarizer $H$ such that

$$
\left\|v_{H}-u^{t}\right\|_{p}<\left\|v-u^{t}\right\|_{p}
$$

It follows that

$$
\begin{aligned}
\left\|\left(u_{m^{\prime}}\right)_{H}-u^{t}\right\|_{p}-\left\|u_{m^{\prime}}-u^{t}\right\|_{p} & \leq\left\|\left(u_{m^{\prime}}\right)_{H}-v_{H}\right\|_{p}+\left\|v_{H}-u^{t}\right\|_{p} \\
+\left\|u_{m^{\prime}}-v\right\|_{p}-\left\|v-u^{t}\right\|_{p} & \leq 2\left\|u_{m^{\prime}}-v\right\|_{p}+\left\|v_{H}-u^{t}\right\|_{p}-\left\|v-u^{t}\right\|_{p} \\
& \rightarrow\left\|v_{H}-u^{t}\right\|_{p}-\left\|v-u^{t}\right\|_{p}<0
\end{aligned}
$$

as $m^{\prime} \rightarrow \infty$.

On the other hand the sequence $\left\|u_{m}-u^{t}\right\|_{p}$ is monotonically decreasing. Hence,

$$
\left\|u_{m+1}-u^{t}\right\|_{p}-\left\|u_{m}-u^{t}\right\|_{p} \rightarrow 0 \quad \text { as } m \rightarrow \infty .
$$

Together with (7.12) this contradicts the minimality property (7.11).

Lemma 7.5. Let $u \in C\left(\mathbb{R}^{n}\right) \cap L^{1}\left(\mathbb{R}^{n}\right)$ and let $u_{m}$ be defined as in Lemma 7.4, whereby the condition (7.11) is satisfied with $p=1$. Then

$$
u_{m} \rightarrow u^{t} \quad \text { in } C\left(\mathbb{R}^{n}\right) .
$$

Proof. By Lemma 7.4, $u_{m} \rightarrow u^{t}$ in $L^{1}\left(\mathbb{R}^{n}\right)$, and the functions $u_{m}$ are equicontinuous in view of Lemma 5.1 [14]. Because of Lemma 6.2 [14] we also have

$$
u_{m^{\prime}} \rightarrow v \quad \text { in } C\left(\mathbb{R}^{n}\right)
$$

for a subsequence $u_{m^{\prime}}$ and $\omega_{v} \leq \omega_{u}$. Thus $v=u^{t}$ and the assertion follows. 


\section{RESCALING AND LiMit CASES}

The $T^{t}$-transformation of sets and functions defined in Section 4 depends on the height $t$ of the moving plane of symmetrization $\{y=t\}$, the range of which is $-\infty<t<\infty$. We recall that in its original setting as described in the Introduction the problem on the continuous symmetrization requires the standard range $0 \leq t \leq 1$ with the "boundary conditions":

$$
T^{0}(\Omega)=\Omega, \quad T^{1}(\Omega)=\Omega^{*},
$$

where $\Omega^{*}$ denotes $(1, n)$-Steiner symmetrization of $\Omega$ with respect to a fixed plane $\left\{y=t_{0}\right\}$. If $t_{0}=1$, then the $T^{t}$-transformation perfectly matches these conditions when restricted to the family $\mathcal{M}_{n}[0,1]$, where

$$
\mathcal{M}_{n}[a, b]:=\left\{\Omega \in \mathcal{M}_{n}: \Omega \subset \Pi(a, b)\right\}
$$

and

$$
\Pi(a, b):=\left\{(x, y) \in \mathbb{R}^{n}: a \leq y \leq b\right\}, \quad-\infty \leq a<b \leq \infty .
$$

If $\Omega \in \mathcal{M}_{n}[a, b]$ with $-\infty<a<b<\infty$, the linear change of variables $\tau=(t-a) /(b-a)$ gives the desired range $0 \leq \tau \leq 1$ with $\tilde{T}^{0}(\Omega)=\Omega$ and $\tilde{T}^{1}(\Omega)=\Omega^{*}$, where $\tilde{T}^{\tau}=T^{t}$ and the asterisk denotes the $(1, n)$-Steiner symmetrization with respect to $\{y=b\}$. The case $\Omega \in \mathcal{M}_{n}[-\infty, b]$ with $b<\infty$ can be handled in a similar way with a non-linear change of variables, for instance, $\tau=(1+b-t)^{-1}$.

The remaining case $\Omega \in \mathcal{M}_{n}[a, \infty]$ with $-\infty \leq a<\infty$ requires some additional consideration since the limit set $\Omega^{\infty}$ is not defined yet. One possible way to "correct" this defect is to use a post-composition $\widehat{T}^{t}=S h^{t} \circ T^{t}$ of the $T^{t}$-transformation with a continuous shift $S h^{t}$ of $\left(t-t_{0}\right)^{+}$units in the direction of the negative $y$-axis. Here $\left(t-t_{0}\right)^{+}=\max \left\{0, t-t_{0}\right\}$. In this case, the limit set $\Omega^{\infty}=\widehat{T}^{\infty}(\Omega)$ may be identified with the Steiner symmetrization $\Omega^{*}$ of $\Omega$ with respect to the plane $\left\{y=t_{0}\right\}$. Of course, $\widehat{T}^{t}$ still possesses all basic properties of the $T^{t}$-transformation. In particular, Lemmas 4.4 and 5.1 are still valid for $\widehat{T}^{t}$ for all $-\infty<t \leq+\infty$. The case $t=\infty$ is included here because $\widehat{T}^{\infty}$ is just the Steiner symmetrization with respect to $\{y=$ $\left.t_{0}\right\}$. Changing the variables via $\tau=(1 / \pi) \arctan t+1 / 2$ we will have a family of transformations $\widetilde{T}^{\tau}$ defined on the standard interval $I=\{\tau: 0 \leq \tau \leq 1\}$. As we have seen in previous sections, the $T^{t}$-transformations is continuous from the left for $-\infty<t<\infty$. Therefore the $\widetilde{T}^{t}$-transformation is continuous from the left on the open interval $0<\tau<1$. By this reason, the $\widetilde{T}^{t}$-transformation will be called the continuous symmetrization defined on the standard interval.

Being restricted to the class of bounded sets and functions with bounded supports, the $\widetilde{T}^{t}$-transformation is continuous at $\tau=1$ as well. For unbounded sets and functions with unbounded supports the situation is different: some functionals of domains or functions are continuous at $\tau=1$ while some other such functionals are not continuous at $\tau=1$. For instance, Lemma 5.1 is obviously not valid if $\Omega$ is an unbounded set while the relevant Steiner symmetrization of $\Omega$ is bounded.

To define a one-dimensional $T^{t}$-transformation for an arbitrary half-space $H=H(a, n)$, we may combine the $T^{t}$-transformation of Definition 4.3 with appropriate affine transformations of $\mathbb{R}^{n}$. To be more precise, let $k>0$ and let $A$ be an orthogonal $n \times n$ matrix such that the operator $L:=k(A-A a)$ maps $H(a, n)$ onto $\{y>0\}$. Then $T_{H}^{t}:=L^{-1} \circ T^{t} \circ L$ is the desired continuous symmetrization with respect to $H$. If 
$M \in \mathcal{M}_{n}$ is bounded then the scaling factor $k$ can be chosen such that $L(M) \subset B^{(n)}$ and therefore when working with bounded sets $M$ we may assume without loss of generality that $M \subset B^{(n)}$. Similarly if $u \in \mathcal{S}_{n}$ has a bounded support we often assume without loss of generality that $\operatorname{supp} u \subset B^{(n)}$.

We stress once more that any $T_{H}^{t}$-transformation can be rescaled to get a $\widetilde{T}_{H}^{t}$-transformation defined on the standard interval $I$ and that all basic properties of continuous symmetrization discussed in Sections 4-7 still remain true for any $\widetilde{T}_{H}^{t}$-transformation.

\section{Continuous $(k, n)$-Steiner Symmetrizations}

To define a one-parameter family of rearrangements $T_{k}^{t}$ left continuous in $t \in[0,1]$ transforming sets and functions into their $(k, n)$-Steiner symmetrization for any $2 \leq$ $k \leq n$, we suggest the following inductive algorithm:

Let $2 \leq k \leq n$. Suppose that for any $(n-k+1)$-dimensional plane $L^{\prime}$ we have defined a continuous family of rearrangements transforming sets, functions, etc. into their $(k-1, n)$-Steiner symmetrizations with respect to $L^{\prime}$. Then let $S$ be a $(k, n)$-Steiner symmetrization with respect to an $(n-k)$-dimensional plane $L$. By Lemma 3.1, we can choose two intersecting $(n-k+1)$-dimensional planes $L_{1}$ and $L_{2}$ defining $(k-1, n)$ Steiner symmetrizations $S_{1}$ and $S_{2}$ such that

$$
S=\lim _{i \rightarrow \infty}\left(S_{2} \circ S_{1}\right)^{i}=\lim _{i \rightarrow \infty} S_{1} \circ\left(S_{2} \circ S_{1}\right)^{i},
$$

where $\left(S_{2} \circ S_{1}\right)^{0}$ is the identity transformation.

By our inductive assumption there are continuous $(k-1, n)$-Steiner symmetrizations $T^{1, t}$ and $T^{2, t}, t \in[0,1]$ into $S_{1}$ and $S_{2}$, respectively. Let $t(j)=(j-1) / j$ and let $I_{j}=$ $\{t: t(j) \leq t<t(j+1)\}, j=1,2, \ldots$ Then $\left(\cup_{j=1}^{\infty} I_{j}\right) \cup\{1\}$ is a disjoint decomposition of the standard interval $I$. Next, for every $j=1,2, \ldots$ and every $t \in I_{j}$ we define the transformation $T_{k}^{t}$ by

$$
T_{k}^{t}=\left\{\begin{array}{lll}
T^{1, \tau} \circ\left(S_{2} \circ S_{1}\right)^{i-1} & \text { with } \quad \tau=\frac{t-t(2 i-1)}{t(2 i)-t(2 i-1)} & \text { if } j=2 i-1, \\
T^{2, \tau} \circ S_{1} \circ\left(S_{2} \circ S_{1}\right)^{i-1} & \text { with } \quad \tau=\frac{t-t(2 i)}{t(2 i+1)-t(2 i)} & \text { if } j=2 i,
\end{array}\right.
$$

where $i=1,2, \ldots$

Inductive algorithm (9.2) defines a continuous transformation into a $(k, n)$-Steiner symmetrization $S$ as follows. First, we choose a complete binary tree $\mathcal{T}$ of planes $L_{0 i_{1} \ldots i_{l}}, 1 \leq l \leq k-1, i_{s} \in\{1,2\}$, rooted at $L=L_{0}$ such that for every $1 \leq l \leq k-2$ and every multi-index $i_{1} \ldots i_{l}$, the planes $L_{0 i_{1} \ldots i_{1} 1}$ and $L_{0 i_{1} \ldots i_{l} 2}$ are $(n-k+l+1)$ dimensional and the corresponding $(k-l-1, n)$-Steiner symmetrizations $S_{0 i_{1} \ldots i_{l} 1}$ and $S_{0 i_{1} \ldots i_{l} 2}$ approximate $S_{0 i_{1} \ldots i_{l}}$ as in (9.1).

Definition 9.1. By $S C(k, n)$-Steiner symmetrization corresponding to a tree $\mathcal{T}$ we mean a transformation $T_{k}^{t}$ of sets and functions defined inductively by (9.2), where the initial $2^{k-1}$ continuous 1-dimensional symmetrizations are selected to be $S C 1$ - symmetrizations with respect to the corresponding hyperplanes $L_{0 i_{1} \ldots i_{k-1}}$, where $i_{1} \ldots i_{k-1} \in$ $\{1,2\}^{k-1}$. 
Three remarks are in order now:

Remark 9.1. The Sarvas approximation scheme used in the algorithm (9.2) may be replaced by any other approximation scheme. For example, one may use a sequence $S_{i}, i=1,2, \ldots$ of $(1, n)$ - Steiner symmetrizations to approximate a given $(k, n)$-Steiner symmetrization: $S=\lim _{n \rightarrow \infty} \bigcirc_{i=1}^{n} S_{i}$. In this case a $T^{t}$-transformation can be defined as $T^{t}=T_{n}^{\tau} \circ\left(\bigcirc_{i=1}^{n} S_{i}\right)$ with $\tau=(t-t(i)) /(t(i+1)-t(i))$.

Remark 9.2. One can define a continuous $(k, n)$-symmetrization by replacing Solynin's one dimensional symmetrization with Brock's one dimensional symmetrization. Alternatively, one may want to define a continuous $(k, n)$-symmetrization by "mixing" in the different stages of the algorithm (9.2) the SC symmetrizations with BC symmetrizations (or with other types of continuous $(1, n)$-symmetrization if those will be discovered).

Remark 9.3. Any continuous deformation defined by the algorithm (9.2) is generated by two kinds of transformations, continuous one dimensional symmetrization and $(k, n)$-Steiner symmetrization. Such a "hybrid" will inherit the properties that both its "parents" have and often the proof of a particular property of the transformation $T_{k}^{t}$, for any fixed $t$, can be given via what we call the "standard inductive argument". More precisely the latter means that if a certain statement is known to be true for any $(k, n)$-Steiner symmetrization, as well as for a corresponding continuous one dimensional symmetrization, and if the corresponding property is invariant under translations and scaling, then the corresponding result will remain true for any continuous $k$-dimensional symmetrization defined by the algorithm (9.2). We will demonstrate how this "standard inductive argument" works in the proof of Theorem 9.1 below. We want to emphasize here that if the assumptions of some theorem or lemma deal with the varying parameter $t$, then the standard inductive argument is not valid and the proof of such statement is, usually, not so straightforward, cf. the proof of Theorem 9.2.

Theorem 9.1. Let $T_{k}^{t}$ be an $S C(k, n)$-Steiner symmetrization into a $(k, n)$ - Steiner symmetrization corresponding to the decomposition $\mathbb{R}^{n}=\mathbb{R}^{n-k} \times \mathbb{R}^{k}$ and let $T_{k, x_{0}^{\prime}}^{t}$ be a restriction of $T_{k}^{t}$ onto the slice $\mathbb{R}\left(x_{0}^{\prime}\right):=\left\{x=\left(x_{0}^{\prime}, y\right): y \in \mathbb{R}^{k}\right\}$. Then $T_{k}^{t}$, respectively $T_{k, x_{0}^{\prime}}^{t}$, acts on $\mathbb{R}^{n}$, respectively on $\mathbb{R}\left(x_{0}^{\prime}\right)$, as an open, compact, and smoothing rearrangement, which is continuous from the inside and from the outside.

Proof. The result is already known for the one dimensional symmetrization, see Lemma 4.4 .

Assume now that the theorem is true for any integer $n \geq 3$ and some $k-1$ such that $2 \leq k<n$. Now for a fixed $t, 0 \leq t \leq 1$, let $T_{k}^{t}$ be a continuous symmetrization as described in the formulation of the theorem. If $t=1$ or $t=t(j)$ for some $j \geq 2$, then the $T_{k}^{t}$-transformation coincides with the $(k, n)$-Steiner symmetrization or it coincides with a finite composition of $(k-1, n)$-Steiner symmetrizations, respectively. In both cases the theorem is known to be true.

Let $t(j)<t<t(j+1)$ for some integer $j \geq 2$. Then according to the algorithm 9.2, the $T_{k}^{t}$-transformation can be represented as a finite composition of $(k-1, n)$-Steiner symmetrizations postcomposed by a continuous $(k-1, n)$-Steiner symmetrization. Then using the known result for $(k-1, n)$-Steiner symmetrizations and the inductive assumption on the continuous $(k-1, n)$-Steiner symmetrizations we conclude that the theorem is true for any $k, 1 \leq k \leq n$. 
The following lemma shows that continuous symmetrizations are monotone in measure on slices. Its proof easily follows from Theorem 9.1 and the non-expansivity property of Lemma 3.1.

Lemma 9.1. Let $T_{k}^{t}$ be a continuous symmetrization as in Theorem 9.1 and let $\Omega \in$ $\mathcal{G}_{b} \cup \mathcal{F}$. If $0 \leq t_{1}<t_{2}<t_{3} \leq 1$, then

$$
\mathcal{L}^{k}\left(\Omega^{t_{2}}\left(x^{\prime}\right) \triangle \Omega^{t_{3}}\left(x^{\prime}\right)\right) \leq \mathcal{L}^{k}\left(\Omega^{t_{1}}\left(x^{\prime}\right) \triangle \Omega^{t_{3}}\left(x^{\prime}\right)\right)
$$

for every $x^{\prime} \in \mathbb{R}^{n-k}$ and therefore

$$
\mathcal{L}^{n}\left(\Omega^{t_{2}} \triangle \Omega^{t_{3}}\right) \leq \mathcal{L}^{n}\left(\Omega^{t_{1}} \triangle \Omega^{t_{3}}\right) .
$$

Theorem 9.2. Let $T_{k}^{t}$ be a continuous symmetrization as in Theorem 9.1 and let $t_{s}$, $s=1,2, \ldots$ be an increasing sequence such that $t_{s} \in[0,1]$ and $t_{s} \rightarrow t_{0}$. Then

$$
d\left(\partial \Omega^{t_{s}}, \partial \Omega^{t_{0}}\right) \rightarrow 0 \quad \text { as } s \rightarrow \infty
$$

if $\Omega$ is a bounded open set and

$$
d\left(\Omega^{t_{s}}, \Omega^{t_{0}}\right) \rightarrow 0 \quad \text { as } s \rightarrow \infty
$$

if $\Omega$ is a compact set.

Proof. To be specific, we will assume that $\Omega$ is a bounded open set. In the case of compact sets the proof is easier and is left to the reader.

We proceed by induction. For $k=1$, the statement is true by Lemma 5.1 modulo some remarks in Section 8. Suppose that for every SC $(j, n)$-Steiner symmetrization with $1 \leq j \leq k-1$ the conclusion of the theorem is true. Our goal now is to prove that the theorem is true for $j=k$.

If $t_{0}<1$, then $t(m)<t_{0} \leq t(m+1)$ for some positive integer $m$. By Definition 9.1 and Algorithm (9.2), the set $\Omega^{t(m+1)}$ is the image of $\Omega^{t(m)}$ under some $(k-1, n)$ Steiner symmetrization $S_{1}$ and moreover there exists a continuous $(k-1, n)$-Steiner symmetrization $T^{1, \tau}$ into $S_{1}$ such that

$$
\Omega^{t}=T^{1, \tau}\left(\Omega^{t(m)}\right) \text { for all } t(m)<t \leq t(m+1),
$$

where $\tau=\tau(t)=(t-t(m)) /(t(m+1)-t(m))$. Since $\tau\left(t_{s}\right) \rightarrow \tau\left(t_{0}\right)$ as $s \rightarrow \infty$, the theorem follows from (9.7) and the inductive assumption.

In the case $t_{0}=1$ the proof is by contradiction. If (9.5) does not hold, then as in the proof of Lemma 5.1 we can find $\varepsilon>0$ and, if necessary, subsequences (for which we keep previous notation), $t_{s}$ and $\Omega_{s}=\Omega^{t_{s}}$, and a sequence of points $x_{s} \in \mathbb{R}^{n}$ with $x_{s} \rightarrow x_{0} \in \mathbb{R}^{n}$ such that one of the following two conditions is satisfied:

(a) $x_{s} \in \partial \Omega^{*}$ and $\operatorname{dist}\left(x_{s}, \partial \Omega_{s}\right) \geq \varepsilon$ for all $s=0,1,2, \ldots$

(b) $x_{s} \in \partial \Omega_{s}$ for $s=1,2, \ldots$ and $\operatorname{dist}\left(x_{s}, \partial \Omega^{*}\right) \geq \varepsilon$ for all $s=0,1,2, \ldots$

In the case (a) we consider two subcases:

(i) $B_{\varepsilon}^{(n)}\left(x_{0}\right) \cap \Omega^{t_{s}}=\emptyset$ for all $s=1,2, \ldots$

(ii) $B_{\varepsilon}^{(n)}\left(x_{0}\right) \subset \Omega^{t_{s}} \quad$ for all $s=1,2, \ldots$ 
Case (i). Let $t\left(m_{s}\right)<t_{s} \leq t\left(m_{s}+1\right)$. In the case under consideration there is $\delta_{0}>0$ such that

$$
\mathcal{L}^{n}\left(\Omega^{t_{s}} \triangle \Omega^{*}\right) \geq \delta_{0}
$$

for all $s=1,2, \ldots$ Since

$$
\Omega^{t\left(m_{s}\right)} \triangle \Omega^{t\left(m_{s}+1\right)} \subset\left(\Omega^{t\left(m_{s}\right)} \triangle \Omega^{*}\right) \cup\left(\Omega^{t\left(m_{s}+1\right)} \triangle \Omega^{*}\right),
$$

by equation 3.11 of Theorem 3.1 we have

$$
\mathcal{L}^{n}\left(\Omega^{t\left(m_{s}\right)} \triangle \Omega^{t\left(m_{s}+1\right)}\right) \leq \mathcal{L}^{n}\left(\Omega^{t\left(m_{s}\right)} \triangle \Omega^{*}\right)+\mathcal{L}^{n}\left(\Omega^{t\left(m_{s}+1\right)} \triangle \Omega^{*}\right) \rightarrow 0
$$

as $m \rightarrow \infty$. Next by Lemma 9.1 ,

$$
\mathcal{L}^{n}\left(\Omega^{t_{s}} \triangle \Omega^{t\left(m_{s}+1\right)}\right) \leq \mathcal{L}^{n}\left(\Omega^{t\left(m_{s}\right)} \triangle \Omega^{t\left(m_{s}+1\right)}\right),
$$

which together with (9.9) contradicts (9.8).

Case (ii). Let $x_{0}=\left(x_{0}^{\prime}, y_{0}\right)$, where $x_{0}^{\prime} \in \mathbb{R}^{n-k}$. Let $\Omega^{*}\left(x_{0}^{\prime}\right)$ and $\Omega^{t}\left(x_{0}^{\prime}\right)$ denote the restrictions of $\Omega^{*}$ and $\Omega^{t}$ respectively in the slice $\mathbb{R}_{x_{0}^{\prime}}$, each of which is not empty by the condition (ii). Since $\Omega^{*}\left(x_{0}^{\prime}\right)$ is a $(k, k)$-Steiner symmetrization of $\Omega\left(x_{0}^{\prime}\right)$, it is a nonempty $k$-dimensional ball. By assumption (a), $x_{0} \in \partial \Omega^{*}$ and therefore

$$
\mathcal{L}^{k}\left(B_{\varepsilon}^{(k)}\left(y_{0}\right) \backslash \Omega^{*}\left(x_{0}^{\prime}\right)\right) \geq \delta_{1}
$$

for some $\delta_{1}>0$. Hence,

$$
\mathcal{L}^{k}\left(\Omega^{*}\left(x_{0}^{\prime}\right) \triangle \Omega^{t_{s}}\left(x_{0}^{\prime}\right)\right) \geq \delta_{1}, \quad s=1,2, \ldots
$$

By inequality (9.3) of Lemma 9.1 we have

$$
\mathcal{L}^{k}\left(\Omega^{t_{s}}\left(x_{0}^{\prime}\right) \triangle \Omega^{t\left(m_{s}+1\right)}\left(x_{0}^{\prime}\right)\right) \leq \mathcal{L}^{k}\left(\Omega^{t\left(m_{s}\right)}\left(x_{0}^{\prime}\right) \triangle \Omega^{t\left(m_{s}+1\right)}\left(x_{0}^{\prime}\right)\right) .
$$

By equation 3.11 of Theorem 3.1,

$$
\mathcal{L}^{k}\left(\Omega^{t(m)}\left(x_{0}^{\prime}\right) \triangle \Omega^{*}\left(x_{0}^{\prime}\right)\right) \rightarrow 0 \quad \text { as } \quad m \rightarrow \infty .
$$

As in the case (i), the latter two relations contradict (9.10). This completes the proof in the case (a).

Now we turn to the condition (b). In this case equation 3.10 of Theorem 3.1 implies that there is a neighborhood $\mathcal{U}$ of $x_{0}$ such that one of the following two conditions is satisfied:

(i) $\mathcal{U} \cap \Omega^{*}=\emptyset$ and $\mathcal{U} \cap \Omega^{t(m)}=\emptyset$ for all $m$ large enough,

(ii) $\mathcal{U} \subset \Omega^{*}$ and $\mathcal{U} \subset \Omega^{t(m)}$ for all $m$ large enough.

For $\delta>0, \tau>0$, and $x_{0}=\left(x_{0}^{\prime}, y_{0}\right)$ with $y_{0} \in \mathbb{R}^{k}$, let

$$
Q_{\delta}\left(x_{0}\right)=B_{\delta}^{(n-k)}\left(x_{0}^{\prime}\right) \times \mathbb{R}^{k}, \quad Q\left(x_{0}, \delta, \tau\right)=B_{\delta}^{(n-k)}\left(x_{0}^{\prime}\right) \times B_{\tau}^{(k)} .
$$

Considering case (i), we first assume that $\left|y_{0}\right|>0$. Since for every $x^{\prime} \in \mathbb{R}^{n-k}$ the section $\Omega^{*}\left(x^{\prime}\right)$ is a ball in $\mathbb{R}^{k}$ centered at the origin, condition (i) implies that we can find $\delta_{0}>0$ and $\tau_{0}>0$ sufficiently small such that

$$
\Omega^{*} \cap Q_{\delta_{0}}\left(x_{0}\right) \subset Q\left(x_{0}, \delta_{0},\left|y_{0}\right|-\tau_{0}\right) \text { and } \Omega_{m} \cap Q_{\delta_{0}}\left(x_{0}\right) \subset Q\left(x_{0}, \delta_{0},\left|y_{0}\right|-\tau_{0}\right)
$$

for all $m$ large enough. According to (9.2) the set $\Omega^{t_{s}}$ is obtained as a result of the action of a certain continuous $(k-1, n)$-Steiner symmetrization of the set $\Omega^{t\left(m_{k}\right)}$ into 
the set $\Omega^{t\left(m_{s}+1\right)}$. Since the intersections $\Omega^{t\left(m_{k}\right)} \cap Q_{\delta_{0}}\left(x_{0}\right)$ and $\Omega^{t\left(m_{k}+1\right)} \cap Q_{\delta_{0}}\left(x_{0}\right)$ both belong to $Q\left(x_{0}, \delta_{0},\left|y_{0}\right|-\tau_{0}\right)$ and since the set $Q\left(x_{0}, \delta_{0},\left|y_{0}\right|-\tau_{0}\right)$ is invariant under the continuous $(k-1, n)$-Steiner symmetrization mentioned above, it follows from the property of monotonicity in slices of the continuous symmetrization that $\Omega^{t_{s}} \cap Q_{\delta_{0}}\left(x_{0}\right) \subset Q\left(x_{0}, \delta_{0},\left|y_{0}\right|-\tau_{0}\right)$ for all $s$ sufficiently large. The latter contradicts the assumptions $x_{s} \in \partial \Omega^{t_{s}}$ and $x_{s} \rightarrow x_{0}$.

If $y_{0}=0$ then, in the case (i), $\Omega^{*} \cap Q_{\delta_{0}}\left(x_{0}\right)=\emptyset$ for some $\delta_{0}>0$. Hence the intersection $\Omega \cap Q_{\delta_{0}}\left(x_{0}\right)$ is also empty and therefore $\Omega^{t_{s}} \cap Q_{\delta_{0}}\left(x_{0}\right)=\emptyset$ for all $s$, which again contradicts the assumptions $x_{s} \in \partial \Omega^{t_{s}}$ and $x_{s} \rightarrow x_{0}$. This finishes the proof in the case (i).

In the case (ii), we argue as above to conclude that there are $\delta_{0}>0$ and $\tau_{0}>0$ such that

$$
Q\left(x_{0}, \delta_{0},\left|y_{0}\right|+\tau_{0}\right) \subset \Omega^{*} \cap Q_{\delta_{0}}\left(x_{0}\right) \quad \text { and } \quad Q\left(x_{0}, \delta_{0},\left|y_{0}\right|+\tau_{0}\right) \subset \Omega_{m} \cap Q_{\delta_{0}}\left(x_{0}\right)
$$

for all $m$ sufficiently large. As above, the monotonicity property of the continuous $(k-1, n)$-Steiner symmetrization from $\Omega^{t\left(m_{s}\right)}$ into $\Omega^{t\left(m_{s}+1\right)}$ shows that $Q\left(x_{0}, \delta_{0}, \tau_{0}\right) \subset$ $\Omega^{t_{s}}$ for all $s$ large enough. The latter contradicts the assumptions $x_{s} \in \partial \Omega^{t_{s}}$ and $x_{s} \rightarrow x_{0}$. The proof of the theorem is complete.

Lemma 9.2. Let $\Omega, \Omega^{\prime} \in \mathcal{G}_{n, b}$ be such that $\overline{\Omega^{\prime}} \subset \Omega$ and let $(\cdot)^{t}$ denote an SC symmetrization into the $(k, n)$-Steiner symmetrization Sym corresponding to the decomposition $\mathbb{R}^{n}=\mathbb{R}^{n-k} \times \mathbb{R}^{k}$. Then for every $t$, there exists a finite number of transformations $P_{1}, \ldots, P_{N}$ such that

$$
\Omega_{N}^{\prime}:=P_{1} \circ \cdots \circ P_{N}\left(\Omega^{\prime}\right) \subset \Omega^{t}
$$

and

$$
\operatorname{dist}\left(\partial \Omega^{\prime}, \partial \Omega\right) \leq \operatorname{dist}\left(\partial \Omega_{N}^{\prime}, \partial \Omega^{t}\right),
$$

where each $P_{j}$ is either a polarization with the polarizer $H_{j}$ such that $\Sigma_{j}:=\partial H_{j} \supset \mathbb{R}^{n-k}$ or a shift in a direction $\vec{l}$ such that $\vec{l} \perp \mathbb{R}^{n-k}$.

Proof. If $k=1$, then the considered symmetrization is one dimensional and the lemma follows from Corollary 7.1.

Let $k \geq 2$. If $t=1$ or $t=t(j)$ for some $j \geq 2$, then the $T_{k}^{t}$-transformation coincides with the $(k, n)$-Steiner symmetrization or with a finite composition of $(k-1, n)$-Steiner symmetrizations. In either case the result is known to be true, cf. Lemmas 7.1 and 7.2 [14]. Therefore for any fixed $t$, the desired conclusion follows via the standard inductive argument as we explained in Remark 9.3 and demonstrated in the proof of Theorem 9.1.

\section{INTEGRAL INEQUALITIES}

Many integral inequalities well-known in the theory of symmetrization remain valid for the continuous symmetrization as well. We start with the following $L^{p}$-continuity lemma.

Lemma 10.1. Let $u \in L_{0+}^{p}\left(\mathbb{R}^{n}\right), 1 \leq p<\infty$ and let $u^{t}=T^{t}(u), 0 \leq t \leq 1$ denote a continuous $(k, n)$-Steiner symmetrization. Then the mapping $t \mapsto u^{t}$ is continuous in $L^{p}$-norm; i.e.,

$$
\lim _{t \rightarrow 0}\left\|u^{s+t}-u^{s}\right\|_{p}=0 \quad \text { for all } \quad 0 \leq s \leq 1
$$


Proof. First we assume that $u$ is a step function. Then

$$
u=\delta \sum_{i=1}^{m} \chi\left(E_{i}\right)
$$

with some $\delta>0$ and some measurable sets $E_{1} \supseteq \ldots \supseteq E_{m}$. Let $E_{i}^{\tau}=T^{\tau}\left(E_{i}\right)$, $0 \leq \tau \leq 1$. By Lemma 9.1, we conclude that

$$
\left\|u^{s+t}-u^{s}\right\|_{p} \leq \delta \sum_{i=1}^{m} \mathcal{L}^{n}\left(E_{i}^{t+s} \Delta E_{i}^{s}\right) \rightarrow 0 \quad \text { as } t \rightarrow 0 .
$$

In general, $u$ can be approximated in the $L^{p}$-norm by step functions $u_{m}$. Then using the inequality

$$
\left\|u^{s+t}-u^{s}\right\| \leq\left\|u^{s+t}-u_{m}^{s+t}\right\|+\left\|u_{m}^{s+t}-u_{m}^{s}\right\|+\left\|u_{m}^{s}-u^{s}\right\|
$$

and the non-expansivity Lemma 3.1 we obtain (10.1).

Our next lemma shows that a similar continuity property remains valid for the space of continuous functions.

Lemma 10.2. Let $u \in C_{0}\left(\mathbb{R}^{n}\right) \cap L_{+}^{1}\left(\mathbb{R}^{n}\right)$. Then for every $0 \leq s \leq 1,\left\|u^{s+t}-u^{s}\right\|_{\infty} \rightarrow 0$ as $t \rightarrow 0$.

Proof. Fix $0 \leq s \leq 1$ and $\varepsilon>0$. Since $u \in C_{0}\left(\mathbb{R}^{n}\right) \cap L_{+}^{1}\left(\mathbb{R}^{n}\right)$, we can find $v \in$ $W_{0}^{1, \infty}\left(\mathbb{R}^{n}\right) \cap L_{+}^{1}\left(\mathbb{R}^{n}\right)$ such that $\|v-u\|_{\infty}<\varepsilon / 3$. Then using the non-expansivity property of Lemma 3.1 we obtain for all $t$ small enough

$$
\begin{aligned}
\left\|u^{s}-u^{s+t}\right\|_{\infty} & \leq\left\|u^{s}-v^{s}\right\|_{\infty}+\left\|v^{s}-v^{s+t}\right\|_{\infty}+\left\|v^{s+t}-u^{s+t}\right\|_{\infty} \\
& \leq 2\|u-v\|_{\infty}+\left\|v^{s}-v^{s+t}\right\|_{\infty}<\varepsilon,
\end{aligned}
$$

which proves the lemma.

The rest of this section contains seven theorems. The first six of them are monotonicity statements that correspond to the inequalities, which are well-known in the theory of Steiner symmetrization. Their proofs in all cases follow the same scheme. First we use an approximation by polarizations to prove the required monotonicity for the continuous SC 1-symmetrization. Then we apply the standard inductive argument to show that the same kind of monotonicity holds for the continuous $(k, n)$-symmetrization for any $k$.

Theorem 10.1 below is related to the well-known convolution type inequalities, cf. [14, Lemmas 8.1, 8.2], [8], [6, Corollary 2]. It shows that convolutions are, in fact, monotone functions of the parameter of the corresponding continuous symmetrization.

Theorem 10.1. Let $u, v, w \in \mathcal{S}_{+}$with $w=w^{*}$, where ()$^{*}$ denotes a $(k, n)$-Steiner symmetrization and let $j$ be a Young-function. Then the integral

$$
\iint_{\mathbb{R}^{2 n}} j\left(\left|u^{t}(x)-v^{t}(y)\right|\right) w(x-y) d x d y
$$

decreases in $0 \leq t \leq 1$ provided that the integral converges for $t=0$.. 
Proof. First we prove the theorem for the SC 1-symmetrization. By the semi-group property (6.7), we have to prove the inequality

$$
\iint_{\mathbb{R}^{2 n}} j\left(\left|u^{t}(x)-v^{t}(x)\right|\right) w(x-y) d x d y \leq \iint_{\mathbb{R}^{2 n}} j(|u(x)-v(x)|) w(x-y) d x d y
$$

for $-\infty<t<\infty$.

First observe that in view of non-expansivity Lemma 3.1, we can restrict ourselves to the case that $u$ and $v$ are continuous functions with bounded support. Then we define inductively two sequences $u_{m}$ and $v_{m}$ of polarizations of $u$ and $v$ as in Lemma 7.4, where the corresponding half-spaces $H_{m}$ are chosen in such a way that the minimality property (7.11) is satisfied. By Lemma 7.5, the sequences $u_{m}$ and $v_{m}$ converge in $C\left(\mathbb{R}^{n}\right)$ to $u^{t}$ and $v^{t}$, respectively. Then (10.2) follows by applying Lemma 8.1 in [14] inductively.

To get values of $t$ varying in the standard range $0 \leq t \leq 1$, we may use scaling and translation as it is explained in Section 8. Of course, the latter two operations do not change the integrals in (10.2).

Finally, it is well known that the desired monotonicity result holds for the $(k, n)$-Steiner symmetrization for any $k, 1 \leq k \leq n$, see [14, Lemma 8.2]. Therefore for $k \geq 2$, the proof of Theorem 10.1 follows via our standard inductive argument.

The Dirichlet-type inequalities for functions and their $(k, n)$-Steiner symmetrizations also admit continuous counterparts.

Theorem 10.2. Let $u \in W_{+}^{1, p}\left(\mathbb{R}^{n}\right) \cap \mathcal{S}_{+}, 1 \leq p \leq+\infty$. Then $u^{t} \in W_{+}^{1, p}\left(\mathbb{R}^{n}\right) \cap \mathcal{S}_{+}$for all $0 \leq t \leq 1$ and $\left\|\nabla u^{t}\right\|_{p}$ decreases in $0 \leq t \leq 1$.

Furthermore, if $V$ is some linear subspace which either contains all " $y$-directions" $x_{n-k+1}, \ldots, x_{n}$, or is orthogonal to each of these directions, then $\left\|\nabla_{V} u^{t}\right\|_{p}$ decreases in $0 \leq t \leq 1$.

Proof. For the $(k, n)$-Steiner symmetrization this result is well known, see [14, Theorem 8.2]. So, we give the proof for the case of the SC 1-symmetrization. Then for $k \geq 2$, Theorem 10.2 will follow via the standard inductive argument.

Let $u^{t}$ denote the SC 1-symmetrization of $u$. The semigroup property (6.7) shows that it is enough to prove the inequalities

$$
\left\|\nabla u^{t}\right\|_{p} \leq\|\nabla\|_{p} \quad \text { and } \quad\left\|\nabla_{V} u^{t}\right\|_{p} \leq\left\|\nabla_{V} u\right\|_{p}
$$

for $-\infty<t<\infty$.

For a fixed $t$, let $u_{m}$ be the sequence of polarizations of $u$ defined by Lemma 7.4, which converges to $u^{t}$ in $L^{p}\left(\mathbb{R}^{n}\right)$. We consider two cases.

(i) Let $1<p<\infty$. Since $\left\|\nabla u_{m}\right\|_{p}=\|\nabla u\|_{p}$, by Lemma 5.3 [14] we can find a function $v \in W^{1, p}\left(\mathbb{R}^{n}\right)$ and a subsequence $u_{m^{\prime}}$ such that

$$
u_{m^{\prime}} \rightarrow v \quad \text { weakly in } W^{1, p}\left(\mathbb{R}^{n}\right) .
$$

This means that for every $\varphi \in C_{0}^{\infty}\left(\mathbb{R}^{n}\right)$ and $i=1, \ldots, n$,

$$
\int_{\mathbb{R}^{n}} \varphi v_{x_{i}} d x \leftarrow \int_{\mathbb{R}^{n}} \varphi \frac{\partial\left(u_{m^{\prime}}\right)}{\partial x_{i}} d x=-\int_{\mathbb{R}^{n}} \varphi_{x_{i}} u_{m^{\prime}} d x \rightarrow-\int_{\mathbb{R}^{n}} \varphi_{x_{i}} u^{t} d x,
$$

that is, $v=u^{t}$. In view of the lower semi-continuity of the norm it follows that

$$
\left\|\nabla u^{t}\right\|_{p} \leq \liminf \left\|\nabla\left(u_{m}\right)\right\|_{p}=\|\nabla u\|_{p}
$$


that is the first inequality in (10.3). Using equation (5.10) of Lemma 5.3 [14] one can prove the second inequality in (10.3) analogously.

(ii) Let $p=1$. By Lemma 5.3 [14], the functions $\left|\nabla u_{m}\right|$ and $|\nabla u|$ are rearrangements of each other. This means that for every $\delta>0$,

$$
\begin{aligned}
\sup \left\{\int_{E}\left|\left(u_{m}\right)_{x_{i}}\right| d x: L^{n}(E) \leq \delta\right\} & \leq \sup \left\{\int_{E}\left|\nabla u_{m}\right| d x: L^{n}(E) \leq \delta\right\} \\
& =\sup \left\{\int_{E}|\nabla v| d x: L^{n}(E) \leq \delta\right\} .
\end{aligned}
$$

Hence, if $E_{k}$ is any sequence of measurable sets with $\lim \left(L^{n}\left(E_{k}\right)\right)=0$, we infer that

$$
\sup \left\{\int_{E_{k}}\left|\left(u_{m}\right)_{x_{i}}\right| d x: m \in \mathbb{N}\right\} \rightarrow 0 \quad \text { as } k \rightarrow \infty .
$$

Applying a well-known weak compactness criterion in $L^{1}\left(\mathbb{R}^{n}\right)$, see [3, p. 199], we again can extract a subsequence $u_{m^{\prime}}$ converging weakly in $W^{1,1}\left(\mathbb{R}^{n}\right)$. Finally, proceeding as in case (i) the assertion follows in the case $p=1$ too.

The following theorem gives an analog of the previous theorem for the spaces of continuous functions.

Theorem 10.3. Let $u \in C\left(\mathbb{R}^{n}\right) \cap \mathcal{S}_{+}$. Then $\omega_{u^{t}}$ decreases in $0 \leq t \leq 1$.

Proof. Let $u \in L_{+}^{1}\left(\mathbb{R}^{n}\right)$. For the SC 1-symmetrization the result follows from the proof of Lemma 7.5. In the general case we choose a sequence $u_{m}$ of functions in $C\left(\mathbb{R}^{n}\right) \cap$ $L_{+}^{1}\left(\mathbb{R}^{n}\right)$ converging to $u$ in $C\left(\mathbb{R}^{n}\right)$. Then applying the non-expansivity Lemma 3.1 we obtain

$$
\left\|\left(u_{m}\right)^{t}-u^{t}\right\|_{\infty} \leq\left\|u_{m}-u\right\|_{\infty}, \quad m=1,2, \ldots
$$

and the assertion for the SC 1-symmetrization follows. For $k \geq 2$, we apply the standard inductive argument.

It is also easy to prove the monotonicity property of convex functionals.

Theorem 10.4. Let $u \in W_{+}^{1,1}\left(\mathbb{R}^{n}\right)$ and let $j$ be a Young-function such that $\int_{\mathbb{R}^{n}} j(|\nabla u|) d x<\infty$. Then the integral mean $\int_{\mathbb{R}^{n}} j\left(\left|\nabla u^{t}\right|\right) d x$ decreases in $0 \leq t \leq 1$.

Furthermore, if $V$ is a linear subspace which either contains all " $y$-directions" $x_{n-k+1}, \ldots, x_{n}$, or is orthogonal to each of these directions, then $\int_{\mathbb{R}^{n}} j\left(\left|\nabla_{V} u^{t}\right|\right) d x$ decreases in $0 \leq t \leq$ 1.

Proof. The standard inductive argument still works. Therefore, it is remains to prove the theorem for the SC 1-symmetrization.

Fix $t,-\infty<t<\infty$. By the semigroup property (6.7), we have to prove the inequalities

$$
\int_{\mathbb{R}^{n}} j\left(\left|\nabla u^{t}\right|\right) d x \leq \int_{\mathbb{R}^{n}} j(|\nabla u|) d x, \quad \int_{\mathbb{R}^{n}} j\left(\left|\nabla_{V} u^{t}\right|\right) d x \leq \int_{\mathbb{R}^{n}} j\left(\left|\nabla_{V} u\right|\right) d x .
$$

Assume first that $u \in C_{0+}^{0,1}\left(\mathbb{R}^{n}\right)$. If we choose a sequence of polarizations of $u$ converging to $u^{t}$ in $L^{1}\left(\mathbb{R}^{n}\right)$, we conclude from Lemma 5.3 [14] that

$$
\int_{\mathbb{R}^{n}} j\left(\left|\nabla u_{m}\right|\right) d x=\int_{\mathbb{R}^{n}} j(|\nabla u|) d x .
$$


Because of the weak lower semi-continuity of the integral functional this leads to

$$
\int_{\mathbb{R}^{n}} j(|\nabla u|) d x=\liminf \int_{\mathbb{R}^{n}} j\left(\left|\nabla u_{m}\right|\right) d x \geq \int_{\mathbb{R}^{n}} j\left(\left|\nabla u^{t}\right|\right) d x .
$$

If $u \in W_{+}^{1,1}\left(\mathbb{R}^{n}\right)$, we choose a sequence $v_{m} \in C_{0+}^{0,1}\left(\mathbb{R}^{n}\right)$ such that

$$
v_{m} \rightarrow u \quad \text { in } W^{1,1}\left(\mathbb{R}^{n}\right)
$$

and

$$
\int_{\mathbb{R}^{n}} j\left(\left|\nabla v_{m}\right|\right) d x \rightarrow \int_{\mathbb{R}^{n}} j(|\nabla u|) d x
$$

This means that we have for a subsequence $v_{m^{\prime}}$

$$
\left(v_{m^{\prime}}\right)^{t} \rightarrow u^{t} \quad \text { weakly in } W^{1,1}\left(\mathbb{R}^{n}\right),
$$

and we conclude by the weak lower semi-continuity of the functionals that the first inequality in (10.4) holds true.

One can prove the second inequality in (10.4) analogously.

An analog of Theorem 10.2 also holds for $B V$-functions.

Theorem 10.5. If $u \in B V\left(\mathbb{R}^{n}\right) \cap L_{+}^{1}\left(\mathbb{R}^{n}\right)$, then $u^{t} \in B V\left(\mathbb{R}^{n}\right) \cap L_{+}^{1}\left(\mathbb{R}^{n}\right)$ and $\left\|D u^{t}\right\|_{B V}$ decreases in $0 \leq t \leq 1$.

Proof. As in the previous theorems, we have to prove the result for the SC 1symmetrization. By the semigroup property (6.7) we have to prove that

$$
\left\|D u^{t}\right\|_{B V} \leq\|D u\|_{B V}
$$

for any fixed $t,-\infty<t<\infty$.

We choose a sequence of functions $u_{m} \in W_{+}^{1,1}\left(\mathbb{R}^{n}\right)$ which converges to $u$ in $B V\left(\mathbb{R}^{n}\right)$. By Lemma 5.3 [14], the functions $\left(u_{m}\right)^{t}$ are equibounded in $W^{1,1}\left(\mathbb{R}^{n}\right)$. Therefore there are some function $v \in B V(\mathbb{R})^{n}$ and a sequence $u_{m^{\prime}}$ such that

$$
\left(u_{m^{\prime}}\right)^{t} \rightarrow v \quad \text { weakly in } B V\left(\mathbb{R}^{n}\right) .
$$

On the other hand from the inequalities

$$
\left\|\left(u_{m}\right)^{t}-u^{t}\right\|_{1} \leq\left\|u_{m}-u\right\|_{1}
$$

we conclude that

$$
\left(u_{m}\right)^{t} \rightarrow u^{t} \quad \text { in } L^{1}\left(\mathbb{R}^{n}\right) .
$$

Now let $\mu_{i}$ denote the Radon-measure which is associated with the weak partial derivative $v_{x_{i}}, i=1, \ldots, n$. Then we have for every $\varphi \in C_{0}^{\infty}\left(\mathbb{R}^{n}\right)$,

$$
\int_{\mathbb{R}^{n}} \varphi d \mu_{i} \leftarrow \int_{\mathbb{R}^{n}} \varphi \frac{\partial\left(\left(u_{m^{\prime}}\right)^{t}\right)}{\partial x_{i}} d x=-\int_{\mathbb{R}^{n}} \varphi_{x_{i}}\left(u_{m^{\prime}}\right)^{t} d x \rightarrow-\int_{\mathbb{R}^{n}} \varphi_{x_{i}} u^{t} d x,
$$

which means that $v=u^{t}$.

Finally, the weak lower semi-continuity of the norm gives

$$
\left\|D u^{t}\right\|_{B V} \leq \liminf \left\|\nabla\left(\left(U_{m}\right)^{t}\right)\right\|_{1}=\lim \left\|\nabla u_{m}\right\|_{1}=\|D u\|_{B V} .
$$

Choosing for $u$ in Theorem 10.5 the characteristic function of a set of finite perimeter we derive the following "monotonic isoperimetric inequality" in $\mathbb{R}^{n}$. 
Theorem 10.6. Let $E$ is a Caccioppoli-set in $\mathbb{R}^{n}$. Then the perimeter $\left\|D \chi_{E^{t}}\right\|_{B V}$ of $E^{t}$ is a decreasing function in $0 \leq t \leq 1$.

Now we prove that the mapping $t \mapsto u^{t}$ is continuous from the left in Sobolev-spaces $W_{+}^{1, p}\left(\mathbb{R}^{n}\right)$. It is worth mentioning here that an analog of this result does not hold in the space $B V\left(\mathbb{R}^{n}\right) \cap L_{+}^{1}\left(\mathbb{R}^{n}\right)$. The characteristic function $u=\chi_{I}$ of a single interval $I \subset \mathbb{R}$ provides a simple counterexample in dimension $n=1$.

Theorem 10.7. Let $u \in W_{+}^{1, p}\left(\mathbb{R}^{n}\right), 1 \leq p<\infty$ and let $t_{m}, m=1,2, \ldots$ be an increasing sequence in $(0,1)$ such that $t_{m} \rightarrow t$ as $m \rightarrow \infty$. Then

$$
u^{t_{m}} \longrightarrow u^{t} \quad \text { in } W^{1, p}\left(\mathbb{R}^{n}\right) \text {. }
$$

Proof. First we consider the case $p>1$. By Lemma 10.1, we have $u^{t_{m}} \rightarrow u^{t}$ in $L^{p}\left(\mathbb{R}^{n}\right)$. From this we conclude that

$$
u^{t_{m}} \rightarrow u^{t} \quad \text { weakly in } W^{1, p}\left(\mathbb{R}^{n}\right) .
$$

In addition, Theorem 10.2 combined with the weak lower semi-continuity of the $L^{p_{-}}$ norm implies

$$
\lim _{m \rightarrow \infty}\left\|\nabla u^{t_{m}}\right\|_{p}=\left\|\nabla u^{t}\right\|_{p} .
$$

Since for $p>1$ the spaces $W^{1, p}\left(\mathbb{R}^{n}\right)$ are uniformly convex, (10.6) follows from (10.7) and (10.8).

Now let $p=1$. We note that (10.7) and (10.8) remain true for $p=1$. Then we fix an index $i \in\{1, \ldots, n\}$ and set $v_{m}:=\left(u^{t_{m}}\right)_{x_{i}}, v:=\left(u^{t}\right)_{x_{i}}$. Let $j(z):=\sqrt{1+z^{2}}-1$. Then $j(z)$ is a Young-function which satisfies the inequality $j(z) \leq z$. Therefore Theorem 10.4 and the weak lower semi-continuity property of the integral imply that

$$
\lim _{m \rightarrow \infty} \int_{\mathbb{R}^{n}}\left(\sqrt{1+v_{m}^{2}}-1\right) d x=\int_{\mathbb{R}^{n}}\left(\sqrt{1+v^{2}}-1\right) d x .
$$

Applying Taylor's formula with a reminder we obtain

$$
\begin{aligned}
\int_{\mathbb{R}^{n}}\left(\sqrt{1+v_{m}^{2}}-1\right) d x & \geq \int_{\mathbb{R}^{n}}\left(\sqrt{1+v^{2}}-1\right) d x+\int_{\mathbb{R}^{n}} \frac{v}{\sqrt{1+v^{2}}}\left(v_{m}-v\right) d x \\
& +\frac{1}{2} \int_{\mathbb{R}^{n}} \frac{\left(v_{m}-v\right)^{2}}{\left(1+c_{m}^{2}\right)^{3 / 2}} d x,
\end{aligned}
$$

where $c_{m}^{2}=\max \left\{v^{2}, v_{m}^{2}\right\}$. Passing to the limit as $m \rightarrow \infty$ we obtain

$$
\lim _{m \rightarrow \infty} \int_{\mathbb{R}^{n}} \frac{\left(v_{m}-v\right)^{2}}{\left(1+c_{m}^{2}\right)^{3 / 2}} d x=0 .
$$

The latter equation implies that for every positive integer $k$,

$$
\lim _{m \rightarrow \infty} \int_{F_{k, m}}\left|v_{m}-v\right| d x=0,
$$

where $F_{k, m}=\left\{x \in \mathbb{R}^{n}:\left|v_{m}\right| \leq k,|v| \leq k\right\}$. Since $v_{m} \rightarrow v$ weakly in $L^{p}\left(\mathbb{R}^{n}\right)$ we find that

$$
\lim _{k \rightarrow \infty} \int_{G_{k, m}}\left|v_{m}\right| d x=0
$$

uniformly over all $m$. Here $G_{k, m}=\left\{x \in \mathbb{R}^{n}:\left|v_{m}\right|>k\right\}$. 
Now (10.6) follows from (10.9) an (10.11) via the inequality

$$
\left\|v_{m}-v\right\|_{1} \leq \int_{F_{k, m}}\left|v_{m}-v\right| d x+2 \int_{G_{k, m}}\left|v_{m}\right| d x+2 \int_{\{|v|>k\}}|v| d x,
$$

which holds for all positive integers $m$ and $k$.

\section{Comparison theOrems}

First we introduce a partial order $\prec^{t}$ related to the continuous $(k, n)$-Steiner symmetrization $T^{t}$.

Definition 11.1. For functions $f, g \in L_{+}^{1}\left(\mathbb{R}^{n}\right)$ and $0 \leq t \leq 1$, we write

$$
f \prec^{t} g \quad \text { if and only if } \quad \int_{\mathbb{R}^{n}} f h d x \leq \int_{\mathbb{R}^{n}} g^{t} h^{t} d x \quad \text { for all } h \in L_{+}^{\infty}\left(\mathbb{R}^{n}\right) .
$$

The following theorem generalizes the well known equivalences in the theory of symmetrization (see [14, Remark 10.1], [4]) to the case of SC 1-symmetrization.

Theorem 11.1. Let $f, g \in L_{+}^{1}\left(\mathbb{R}^{n}\right)$ and let $(\cdot)^{t}$ denote the $S C 1$-symmetrization, $-\infty<$ $t<\infty$. Then the following relations are equivalent:

$$
\begin{aligned}
& f \prec^{t} g \\
& \int_{\mathbb{R}^{n}} f^{t} h^{t} d x \leq \int_{\mathbb{R}^{n}} g^{t} h^{t} d x \quad \text { for all } h \in L_{+}^{\infty}\left(\mathbb{R}^{n}\right), \\
& j(f) \prec^{t} j(g) \quad \text { for all Lipschitz-continuous Young-functions } j, \\
& \int_{2 t-y}^{y} f^{t}\left(x^{\prime}, s\right) d s \leq \int_{2 t-y}^{y} g^{t}\left(x^{\prime}, s\right) d s \quad \text { for all } x^{\prime} \in \mathbb{R}^{n-1} \text { and every } y \geq t, \\
& f^{t}\left(x^{\prime}, y\right) \leq g^{t}\left(x^{\prime}, y\right) \quad \text { for all } x^{\prime} \in \mathbb{R}^{n-1} \text { and every } y \geq t .
\end{aligned}
$$

Proof. (a) To prove that (11.2) implies (11.5), we fix $c>0$ and set $M_{c}:=\{f>c\}$. Then for fixed $x_{0}^{\prime} \in \mathbb{R}^{n-1}$ and positive $\varepsilon$, we define a function

$$
h_{\varepsilon}\left(x^{\prime}, y\right):=\kappa\left(x^{\prime}, y\right) \varphi_{\varepsilon}\left(x^{\prime}, y\right)
$$

with $x=\left(x^{\prime}, y\right) \in \mathbb{R}^{n}$ and

$$
\begin{gathered}
\varphi_{\varepsilon}=\varphi_{\varepsilon}\left(x^{\prime}, y\right):=\chi\left(\left\{\left(x^{\prime}, y\right):\left|x^{\prime}-x_{0}^{\prime}\right|<\varepsilon\right\}\right), \\
\kappa\left(x^{\prime}, y\right):= \begin{cases}1 & \text { if } f\left(x^{\prime}, y\right)>c \text { and } y<y\left(x^{\prime}, t\right) \\
0 & \text { otherwise, }\end{cases}
\end{gathered}
$$

where the separating function $y\left(x_{0}^{\prime}, t\right)=y_{M_{c}}\left(x_{0}^{\prime}, t\right)$ is defined in Section 4 .

Now (11.2) implies

$$
\begin{aligned}
& \int_{\left\{\left|x^{\prime}-x_{0}^{\prime}\right|<\varepsilon\right\}} \int_{2 t-y\left(x^{\prime}, t\right)}^{y\left(x^{\prime}, t\right)} f^{t}\left(x^{\prime}, s\right) d s d x^{\prime}=\int_{\mathbb{R}^{n}} f^{t}\left(h_{\varepsilon}\right)^{t} d x=\int_{\mathbb{R}^{n}} f h_{\varepsilon} d x \leq \\
& \int_{\mathbb{R}^{n}} g^{t}\left(h_{\varepsilon}\right)^{t} d x=\int_{\left\{\left|x^{\prime}-x_{0}^{\prime}\right|<\varepsilon\right\}} \int_{2 t-y\left(x^{\prime}, t\right)}^{y\left(x^{\prime}, t\right)} g^{t}\left(x^{\prime}, s\right) d s d x^{\prime} .
\end{aligned}
$$

Taking the limit here as $\varepsilon \rightarrow 0^{+}$, we obtain

$$
\int_{2 t-y\left(x_{0}^{\prime}, t\right)}^{y\left(x_{0}^{\prime}, t\right)} f^{t}\left(x_{0}^{\prime}, s\right) d s \leq \int_{2 t-y\left(x_{0}^{\prime}, t\right)}^{y\left(x_{0}^{\prime}, t\right)} g^{t}\left(x_{0}^{\prime}, s\right) d s,
$$


where $y\left(x_{0}^{\prime}, t\right)=y_{M_{c}}\left(x_{0}^{\prime}, t\right)$ depends on $c$. Since $c>0$ can be chosen arbitrary small the latter inequality implies (11.5).

(b) To show that (11.2) implies (11.6) we fix $\left(x_{0}^{\prime}, y_{0}\right) \in \mathbb{R}^{n}$ with $y_{0} \geq t$. Then using notation of part (a) of this proof we note that the separating function $y\left(x_{0}^{\prime}, t\right)=$ $y_{M_{c}}\left(x_{0}^{\prime}, t\right)$ depends monotonously on the height of polarization $c$. Therefore we can choose $c>0$ small enough such that $y_{0} \geq y\left(x_{0}^{\prime}, t\right)$. Then we have $f^{t}\left(x_{0}^{\prime}, y\right)=f\left(x_{0}^{\prime}, y\right)$ for all $y \geq y_{0}$. Now we choose the function $h$ in (11.1) to be the Dirac $\delta$-function at $x_{0}=\left(x_{0}^{\prime}, y_{0}\right)$, i.e. $h:=\delta\left(x_{0}\right)$. Then we have $h=h^{t}$. Finally applying (11.1) we derive

$$
f^{t}\left(x_{0}^{\prime}, y_{0}\right)=f\left(x_{0}^{\prime}, y_{0}\right)=\int_{\mathbb{R}^{n}} f h d x \leq \int_{\mathbb{R}^{n}} g^{t} h^{t} d x=g^{t}\left(x_{0}^{\prime}, y_{0}\right),
$$

which is (11.6).

(c) Now we show that (11.5) and (11.6) together imply (11.3). First we assume that $h=\chi(M)$ is a characteristic function of $M \in \mathcal{M}\left(\mathbb{R}^{n}\right)$. Let $y\left(x^{\prime}, t\right)=y_{M}\left(x^{\prime}, t\right)$ and let $M\left(x^{\prime}, t\right)=M\left(x^{\prime}\right) \cap\left\{s: s>y\left(x^{\prime}, t\right)\right\}$. Then (11.5) and (11.6) imply

$$
\begin{aligned}
& \int_{M^{t}} f^{t} d x=\int_{\mathbb{R}^{n-1}}\left\{\int_{2 t-y\left(x^{\prime}, t\right)}^{y\left(x^{\prime}, t\right)} f^{t}\left(x^{\prime}, s\right) d s+\int_{M\left(x^{\prime}, t\right)} f^{t}\left(x^{\prime}, s\right) d s\right\} d x^{\prime} \leq \\
& \int_{\mathbb{R}^{n-1}}\left\{\int_{2 t-y\left(x^{\prime}, t\right)}^{y\left(x^{\prime}, t\right)} g^{t}\left(x^{\prime}, s\right) d s+\int_{M\left(x^{\prime}, t\right)} g^{t}\left(x^{\prime}, s\right) d s\right\} d x^{\prime}=\int_{M^{t}} g^{t} d x .
\end{aligned}
$$

Next let $h$ be a step function, i.e.

$$
h:=\varepsilon \sum_{i=1}^{m} \chi\left(M_{i}\right)
$$

with some $\varepsilon>0$ and some sets $M_{i} \in \mathcal{M}\left(\mathbb{R}^{n}\right)$ such that $M_{1} \supset \cdots \supset M_{m}$. Applying inequality (11.7) to the functions $\chi\left(M_{i}\right)$ we obtain the desired inequality:

$$
\int_{\mathbb{R}^{n}} f^{t} g^{t} d x=\varepsilon \sum_{i=1}^{m} \int_{M_{i}^{t}} f^{t} d x \leq \varepsilon \sum_{i=1}^{m} \int_{M_{i}^{t}} g^{t} d x=\int_{\mathbb{R}^{n}} g^{t} h^{t} d x .
$$

Finally, every $h \in L_{+}^{\infty}\left(\mathbb{R}^{n}\right)$ can be approximated by step functions. Therefore, in the general case (11.3) follows from the previous inequality.

(d) To prove that (11.3) implies (11.4), we may assume without loss of generality that $j \in C^{1}$. For $p \in L_{+}^{\infty}\left(\mathbb{R}^{n}\right)$, we set $h:=j^{\prime}(f) p$. Since $j^{\prime}$ is an increasing function we have $h^{t}=j^{\prime}\left(f^{t}\right) p^{t}$. This equality combined with (11.3) implies

$$
\int_{\mathbb{R}^{n}} f^{t} j^{\prime}\left(f^{t}\right) p^{t} d x \leq \int_{\mathbb{R}^{n}} g^{t} j^{\prime}\left(f^{t}\right) p^{t} d x
$$

Since $j$ is a convex function we have

$$
j\left(g^{t}\right)-j\left(f^{t}\right) \geq j^{\prime}\left(f^{t}\right)\left(g^{t}-f^{t}\right),
$$

which together with (11.8) leads to (11.4).

(e) Finally, (11.2) is a special case of (11.4).

The following corollary shows that equivalencies (11.2) - (11.4) remain valid for continuous $(k, n)$-Steiner symmetrization for all $k, 1 \leq k \leq n$. Its proof follows from Theorem 11.1 via the standard inductive argument. 
Corollary 11.1. Let $f, g \in L_{+}^{1}\left(\mathbb{R}^{n}\right)$ and let $(\cdot)^{t}$, where $0 \leq t \leq 1$, denote the continuous $(k, n)$-Steiner symmetrization. Then equivalencies (11.2) - (11.4) remain valid for all $0 \leq t \leq 1$.

Corollary 11.2. Let $f, g \in L_{+}^{1}\left(\mathbb{R}^{n}\right)$ and let $(\cdot)^{t}$ denote the continuous $(k, n)$-Steiner symmetrization into a given $(k, n)$-Steiner symmetrization $(\cdot)^{*}$. Then for all $s$ and $t$ such that $0 \leq s<t \leq 1$, the following implication holds:

If $f \prec^{s} g$, then $f \prec^{t} g$.

In particular, if $f \prec^{t} g$, then $f \prec^{*} g$.

For the SC 1-symmetrization Corollary 11.2 follows immediately from the semigroup property (6.7). Then for any $k \geq 2$, its proof follows via the standard inductive argument.

Now we prove two comparison lemmas concerning partial symmetry of solutions of certain elliptic and parabolic PDE's. These results and their proofs show, in fact, that the approach to comparison theorems in partially symmetric domains, based on the continuous symmetrization, is a closed relative of the Alexandrov's moving plane method, see [26] and [18]. In the context of comparison theorems the approach based on a continuous symmetrization for the first time was used by Solynin [27].

Lemma 11.1. Let $\Omega \subset \mathbb{R}^{n}$ be a bounded domain, $c \geq 0, f, g \in L_{+}^{2}(\Omega)$, and let $u, v$ be solutions to the following boundary value problems:

$$
u, v \in W_{0}^{1,2}(\Omega), \quad-\Delta u+c u=f, \quad-\Delta v+c v=g \quad \text { in } \Omega .
$$

For $0 \leq t \leq 1$, let $(\cdot)^{t}$ denote a continuous $(k, n)$-Steiner symmetrization. If for some $0 \leq t \leq 1, \Omega=\Omega^{t}, f=f^{t}, g=g^{t}$, and $f \prec^{t} g$, then

$$
u=u^{t}, \quad v=v^{t}
$$

and

$$
u \prec^{t} v .
$$

Proof. First, we prove the lemma for the SC 1-symmetrization. Then, of course, $-\infty<t<\infty$. Equality (11.10) follows easily from the maximum principle.

To prove (11.11), we may assume without loss of generality that $f, g \in C_{0+}^{0,1}(\Omega)$. Then $u$ and $v$ are classical solutions which are smooth in $\Omega$.

Let $G=\left\{x=\left(x^{\prime}, y\right):\left(x^{\prime}, 2 t-y\right) \in \Omega\right\}$ and let $w=u-v$. For $\left(x^{\prime}, y\right) \in G$ and $\varepsilon \geq 0$, we define a function

$$
W_{\varepsilon}\left(x^{\prime}, y\right):=\int_{t}^{y}\left(w\left(x^{\prime}, s\right)+w\left(x^{\prime}, 2 t-s\right)-\sup _{\partial G \cap H_{t}} w-\varepsilon\right) d s .
$$

Since $f \prec^{t} g$ one can easily see that

$$
-\Delta W_{\varepsilon}+c W_{\varepsilon} \leq 0 \quad \text { in } G \text {. }
$$

In addition, we have $W_{\varepsilon}=0$ on $\partial H_{t}$. Since solutions of (11.12) satisfy the maximum principle we have: $\sup _{G} W_{\varepsilon}=\sup _{\partial G \cap H_{t}} W_{\varepsilon}$. Since $\Omega=\Omega^{t}$ one can easily see that the domain $G$ is convex in the direction of $y$-axis. Therefore the positive direction of $y$-axis points outward the domain $G$ on $\partial G \cap H_{t}$. Since $\frac{\partial W_{\varepsilon}}{\partial y} \leq-\varepsilon$ on $\partial G \cap H_{t}$ we conclude that $W_{\varepsilon} \leq 0$ in $G$ for all $\varepsilon>0$. Taking the limit as $\varepsilon \rightarrow 0^{+}$, we obtain

$$
W_{0} \leq 0 \quad \text { in } G \text {. }
$$


Let us show that $w \leq 0$ in $\Omega_{-}(t)$. If not, then $\sup _{\Omega_{-}(t)} w>0$. Since $-\Delta w+c w \leq 0$ in $\Omega_{-}(t)$ and $w=0$ on $\partial \Omega \cap H_{t}$ the maximum principle implies that $\sup _{\Omega_{-}(t)} w=$ $\sup _{\partial H_{t}} w$. The second supremum here is attained at some point $x_{0} \in \partial H_{t}$ with $w\left(x_{0}\right)>$ 0 . Since $w\left(x_{0}\right) \geq \sup _{\partial G \cap H_{t}} w$, the inequality $w\left(x_{0}\right)>0$ implies that $W_{0}(x)>0$ for $x \in G$ in a small neighborhood of $x_{0}$, which contradicts (11.13).

Therefore we have $w \leq 0$ in $\Omega_{-}(t)$. Now (11.11) follows from (11.5), (11.6), and (11.13).

Now for $k \geq 2$, the lemma follows via the standard inductive argument.

Similar lemma holds also for parabolic problems. Its proof follows along the lines of the proof of Lemma 11.1. Therefore the details are left to the reader.

Lemma 11.2. Let $(\cdot)^{s}$ denote a continuous $(k, n)$-Steiner symmetrization, $0 \leq s \leq 1$. Let $c \geq 0, T>0$ and let $\Omega$ be a bounded domain in $\mathbb{R}^{n}$ such that $\Omega=\Omega^{s}$. Let functions $u_{0}, v_{0} \in L_{+}^{2}(\Omega)$ and $f, g \in L_{+}^{2}(\Omega \times(0, T))$ satisfy, respectively, the following conditions:

$$
u_{0}=u_{0}^{s}, \quad v_{0}=v_{0}^{s}, \quad u_{0} \prec^{s} v_{0}
$$

and

$$
f(\cdot, t)=f^{s}(\cdot, t), \quad g=g(\cdot, t)=g^{s}(\cdot, t), \quad f(\cdot, t) \prec^{s} g(\cdot, t) \quad \text { for all } t \in(0, T) .
$$

Further let $u, v \in L^{2}\left(0, T ; W_{0}^{1,2}(\Omega)\right) \cap C\left([0, T] ; L^{2}(\Omega)\right)$ be solutions to the following initial boundary value problems:

$$
\begin{array}{lll}
u_{t}-\Delta u+c u=f, & v_{t}-\Delta v+c v=g, & \text { in } \Omega \times(0, T), \\
u(x, 0)=u_{0}(x), & v(x, 0)=v_{0}(x), & \text { in } \Omega .
\end{array}
$$

Then

$$
u(\cdot, t)=u^{s}(\cdot, t), \quad v(\cdot, t)=v^{s}(\cdot, t) \quad \text { for all } t \in(0, T)
$$

and

$$
u(\cdot, t) \prec^{s} v(\cdot, t) \quad \text { for all } t \in(0, T) .
$$

To simplify some notations in our formulations and proofs, we will use the following definitions from [14]:

Definition 11.2. Let $\Omega \subset \mathbb{R}^{n}$ be a bounded open set, $c \geq 0$, and $f \in L_{+}^{2}(\Omega)$. We say that $u$ solves the problem $\mathbb{B}_{1}(\Omega, c, f)$ if $u$ is the solution to the following boundary value problem:

$$
u \in W_{0}^{1,2}(\Omega), \quad-\Delta u+c u=f \quad \text { in } \Omega .
$$

Definition 11.3. Let $\Omega \subset \mathbb{R}^{n}$ be a bounded open set, $c \geq 0, f \in L_{+}^{2}(\Omega)$ and $\gamma: \mathbb{R}_{0}^{+} \rightarrow$ $\mathbb{R}_{0}^{+}$be a continuous and nondecreasing function. We will say that $\underline{u}$ is a solution of problem $\mathbb{B}_{2}(\Omega, c, \gamma, f)$, if $\underline{u}$ is the nonnegative minimal solution of the following boundary value problem:

$$
u \in W_{0}^{1,2}(\Omega), \quad u \geq 0, \quad-\Delta u+c u=\gamma(u)+f \quad \text { in } \Omega,
$$

that is,

(i) $\underline{u}$ is a solution of the problem (11.18), and

(ii) $0 \leq \underline{u} \leq u$ for all other solutions $u$ of (11.18).

For a brief discussion of important properties of the solutions to the problems in Definitions 11.2 and 11.3, we refer to [14, Section 9]. 
Theorem 11.2. Let $(\cdot)^{t}$ denote a continuous $(k, n)$-Steiner symmetrization, $0 \leq t \leq 1$. Let $\Omega \subset \mathbb{R}^{n}$ be a bounded open set, $c \geq 0, f \in L_{+}^{2}(\Omega)$, and let $\gamma$ be a Young function. For a fixed $t, 0 \leq t \leq 1$, let $g \in L_{+}^{2}\left(\Omega^{t}\right)$ be such that $g^{t}=g$ and $f \prec^{t} g$. Let $u$ and $v$ be the solutions to the problems $B_{2}(\Omega, c, \gamma, f)$ and $B_{2}\left(\Omega^{t}, c, \gamma, g\right)$, respectively. Then

$$
v=v^{t}
$$

and

$$
u \prec^{t} v \text {. }
$$

Proof. As well known, the assertion of this theorem holds true for the $(k, n)$-Steiner symmetrization for any $k, 1 \leq k \leq n$; see, for example, Theorem 10.1 in [14]. Thus, in view of the standard inductive argument we have to prove it for the SC 1 -symmetrization only. Then, of course, $-\infty<t<\infty$.

(1) First we assume that $\gamma \equiv 0$ and that $f$ is a simple function with compact support in $\Omega$, see Definition 7.1. For a fixed $t \in \mathbb{R}$, let $\tilde{v}$ denote the solution to the problem $\mathbb{B}_{1}\left(\Omega^{t}, c, f^{t}\right)$. The maximum principle tells us that $v=v^{t}$ and $\tilde{v}=\tilde{v}^{t}$. Furthermore, let $h$ be an arbitrary function in $L_{+}^{2}\left(\Omega^{t}\right)$ satisfying $h=h^{t}$, and let $w$ be the solution of the problem $\mathbb{B}_{1}\left(\Omega^{t}, c, h\right)$. Since again $w=w^{t}, w \geq 0$, and $f \prec^{t} g$, we find after partial integration that

$$
\int_{\Omega^{t}} \tilde{v} h^{t} d x=\int_{\Omega^{t}} w f^{t} d x \leq \int_{\Omega^{t}} w g d x=\int_{\Omega^{t}} v h^{t} d x
$$

which means that

$$
\tilde{v} \prec^{t} v \text {. }
$$

Next let $\Omega^{\prime}$ be an open set such that $\operatorname{supp}(f) \subset \Omega^{\prime} \subset \overline{\Omega^{\prime}} \subset \Omega$. By Corollary 7.1 and Lemma 7.2, we can find a finite number of polarizations $P_{i}$ with polarizers $H_{i}=\{y>$ $\left.y_{i}\right\}, i=1, \ldots, N$, where

$$
y_{1}<y_{2}<\ldots<y_{N} \leq t,
$$

such that the closure of $\bigcirc_{i=1}^{N} P_{i}\left(\Omega^{\prime}\right)$ is in $\Omega^{t}$ and $\left(\bigcirc_{i=1}^{N} P_{i} f\right)^{t}=f^{t}$.

Let $\Omega_{m}=\bigcirc_{i=1}^{m} P_{i} \Omega^{\prime}$ and $f_{m}=\bigcirc_{i=1}^{m} P_{i} f, m=1, \ldots, N$. Let $u^{\prime}$ and $u_{m}^{\prime}$ be the solutions to the problems $\mathbb{B}_{1}\left(\Omega^{\prime}, c, f\right)$ and $\mathbb{B}_{1}\left(\Omega_{m}^{\prime}, c, f_{m}\right)$, respectively, $m=1, \ldots, N$. Applying Theorem 9.1 [14] we conclude that

$$
u^{\prime} \prec_{H_{1}} u_{1}^{\prime} \prec_{H_{2}} u_{2}^{\prime} \prec_{H_{3}} \cdots \prec_{H_{N}} u_{N}^{\prime},
$$

where " $\prec_{H}$ " denotes the partial order related to the polarization with the polarizer $H$, see [14, p. 1783]. Since $\Omega_{N}^{\prime} \subset \Omega^{t}$ and $f_{N}=f^{t}$, the solutions $u_{N}^{\prime}$ and $\tilde{v}$ satisfy the inequality

$$
0 \leq u_{N}^{\prime} \leq \tilde{v} \quad \text { a.e. in } \Omega_{N} .
$$

Together with (11.21) and (11.22) this implies that $u^{\prime} \prec^{t} v$.

Now we choose open bounded sets $\Omega^{k}$ such that $\overline{\Omega^{k}} \subset \Omega^{k+1}, k=1,2, \ldots$, and $\cup_{k} \Omega^{k}=$ $\Omega$. Let $u^{t}$ denote the solution of the problem $\mathbb{B}_{1}\left(\Omega^{k}, c, f\right), k=1,2, \ldots$ By the above consideration we have $u^{k} \prec^{t} v, k=1,2, \ldots$ By the convergence property of elliptic boundary value problems in varying domains, see Lemma A [14], the sequence $u^{k}$ converges to $u$ in $L^{2}(\Omega)$. This proves the assertion in the case under consideration.

(2) Next we assume that $\gamma \equiv 0$ but $f$ is an arbitrary function in $L_{+}^{2}(\Omega)$. Since simple functions are dense in $L_{+}^{2}(\Omega)$, there is a sequence of simple functions $f^{k}, k=1,2, \ldots$, 
with compact supports in $\Omega$, such that $f^{k} \rightarrow f$ in $L^{2}(\Omega)$. Then we can find open sets $\Omega^{k}, k=1,2, \ldots$, such that $\operatorname{supp}\left(f^{k}\right) \subset \Omega^{k} \subset \overline{\Omega^{k}} \subset \Omega^{k+1} \subset \Omega$ and $\cup_{k} \Omega^{k}=\Omega$.

Let $u^{k}$ and $v_{k}$ be the solutions to the problems $\mathbb{B}_{1}\left(\Omega^{k}, c, f^{k}\right)$ and $\mathbb{B}_{1}\left(\left(\Omega^{k}\right)^{t}, c,\left(f^{k}\right)^{t}\right)$, respectively, $k=1,2, \ldots$. By part (1), we have

$$
u_{k} \prec^{t} v_{k}, \quad k=1,2, \ldots
$$

By Lemma $7.4,\left(f^{k}\right)^{t} \rightarrow f^{t}$ in $L^{2}\left(\Omega^{t}\right)$ and also by the monotonicity property of rearrangements,

$$
\text { closure }\left(\left(\Omega^{k}\right)^{t}\right) \subset\left(\Omega^{k+1}\right)^{t} \subset \Omega^{t} \text { and } \cup_{k}\left(\Omega^{k}\right)^{t}=\Omega^{t} .
$$

Therefore by the convergence property of elliptic boundary value problems in varying domains, see Lemma A [14], we have

$$
u_{k} \rightarrow u \quad \text { in } L^{2}(\Omega) \quad \text { and } v_{k} \rightarrow v \text { in } L^{2}\left(\Omega^{t}\right) .
$$

This together with (11.23) proves the theorem in the case $\gamma \equiv 0$.

(3) Next let $\gamma \not \equiv 0$. According to Remark 9.2 in [14] we approximate $u$ and $v$ by solutions to the problems $\mathbb{B}_{1}\left(\Omega, c, \gamma\left(u_{m-1}\right)+f\right)$ and $\mathbb{B}_{1}\left(\Omega^{t}, c, \gamma\left(v_{m-1}\right)+g\right)$, respectively, $m=1,2, \ldots$. Here $u_{0} \equiv v_{0} \equiv 0$. Assume that we had proved that $u_{m} \prec^{t} v_{m}$ for some $m$. Notice that for $m=0$ this is trivial. Then by equation (11.4) of Theorem 11.1, we obtain that $\gamma\left(u_{m}\right)+f \prec^{t} \gamma\left(v_{m}\right)+g$. By parts (1) and (2) of this proof this means that also $u_{m+1} \prec^{t} v_{m+1}$, and we conclude by induction.

Theorem 11.2 and equivalence relations of Theorem 11.1 lead to the following corollary.

Corollary 11.3. Let $u$ and $v$ be solutions defined in Theorem 11.2. Then for every Young function $j$,

$$
\int_{\Omega} j(u) d x \leq \int_{\Omega^{t}} j(v) d x
$$

if the above integrals converge. In particular,

$$
\|u\|_{p} \leq\|v\|_{p} \quad \text { for all } 1 \leq p \leq \infty .
$$

One might ask under which conditions the equality holds in inequalities (11.24) and (11.25), and believe that equality is possible only — roughly speaking — if $\Omega$ possesses a partial symmetry. For Steiner symmetrizations this result was proved in [14]. To prove this uniqueness result for the continuous $(k, n)$-Steiner symmetrization, we restrict ourselves to the case where $\Omega$ is a domain.

Theorem 11.3. Let $(\cdot)^{t}, 0 \leq t \leq 1$, denote the continuous $(k, n)$-Steiner symmetrization into some $(k, n)$-Steiner symmetrization with respect to a plane $\Sigma$. Let $\Omega, \Omega^{t}, c$, $f, g, \gamma, u$, and $v$ be as in Theorem 11.2 and assume that $\Omega$ is a bounded domain and $f>0$ on $\Omega$. Assume that there is some Lipschitz continuous Young function $j$ which for some $t, 0 \leq t \leq 1$, satisfies

$$
\int_{\Omega} j(u) d x=\int_{\Omega^{t}} j(v) d x>0 .
$$

Then $\Omega=\Omega^{t}$ and $f=g$ modulo some translation in a direction orthogonal to $\Sigma$. 
Proof. For $(k, n)$-Steiner symmetrizations this result is proved in Theorem 10.3 [14]. Thus, thanks to the standard inductive argument, we have to prove the theorem for the SC 1-symmetrization only. Then $-\infty<t<\infty$ and we may assume that $\Sigma=\{y=0\}$. Here $x=\left(x^{\prime}, y\right) \in \mathbb{R}^{n-1} \times \mathbb{R}$.

Assume that for some fixed $t \in \mathbb{R}, \Omega^{t}$ is not a translation of $\Omega$ in the direction of $y$-axis. It follows from Corollary 6.3 that we can find a polarizer $H=\left\{y>t_{0}\right\}$ with $t_{0}<t$ such that either $\left(\Omega_{H}\right)^{t}=\Omega^{t},\left(f_{H}\right)^{t}=f^{t}, \Omega \neq \Omega_{H}$, and $\sigma_{H}(\Omega) \neq \Omega_{H}$ or $\left(\Omega_{H}\right)^{t}=\Omega^{t}$, $\left(f_{H}\right)^{t}=f^{t}, \Omega=\Omega_{H}, f \neq f_{H}$, and $\sigma_{H}(f) \neq f_{H}$. Then, if $w$ is the solution to the problem $\mathbb{B}_{2}\left(\Omega_{H}, c, \gamma, f_{H}\right)$, we conclude by Theorem 9.3 in [14] that

$$
\int_{\Omega} j(u) d x<\int_{\Omega_{H}} j(w) d x .
$$

Further, since $\left(\Omega_{H}\right)^{t}=\Omega^{t}$ and $\left(f_{H}\right)^{t}=f^{t}$, we also have $w \prec^{t} v$. By Corollary 11.3 this means that

$$
\int_{\Omega_{H}} j(w) d x \leq \int_{\Omega^{t}} j(v) d x
$$

which together with (11.27) contradicts (11.26).

Now we assume that for some $t \in \mathbb{R}, \Omega=\Omega^{t}$ modulo translation in the direction of the $y$-axis. Without loss of generality we may assume that $\Omega=\Omega^{t}$. Assume in addition that $f \neq f^{t}$. By Corollary 6.3, there is a polarizer $H=\left\{y>t_{1}\right\}$ with $t_{1}<t$ such that $\Omega_{H}=\Omega$ and $f \neq f_{H}$, and we can argue as before to derive a contradiction to (11.26).

Thus, we have $f=f^{t}$ and it remains to show that $f^{t}=g$.

Assume that $f^{t} \neq g$. We set $\tilde{f}=\gamma(u)+f$ and $\tilde{g}=\gamma(v)+g$. Since $u=u^{t}, v=v^{t}$ and $u \prec^{t} v$, it follows from Theorem 11.1 that

$$
\tilde{f} \prec^{t} \tilde{g}, \quad \tilde{f}=\tilde{f}^{t} \neq \tilde{g}^{t}=\tilde{g},
$$

and also

$$
\int_{2 t-y}^{y}\left(\tilde{f}\left(x^{\prime}, s\right)-\tilde{g}\left(x^{\prime}, s\right)\right) d s \leq 0
$$

for all $x^{\prime} \in \mathbb{R}^{n-1}$ and every $y \geq t$, where the inequality (11.28) must be strict on a subset of $\Omega$ of positive measure. Now let $h$ be an arbitrary function in $L_{+}^{2}(\Omega)$ satisfying $h=h^{t} \not \equiv 0$. Then, if $w$ is the solution to the problem $\mathbb{B}_{1}(\Omega, c, h)$, we conclude that $w=w^{t}$. Moreover, the strong maximum principle yields

$$
\left|\frac{\partial}{\partial y} w(x)\right|>0 \quad \text { a.e. in } \Omega \text {. }
$$

Now after partial integration and by using (11.28) and (11.29) we obtain

$$
\begin{aligned}
\int_{\Omega}(u-v) h d x & =\int_{\Omega} w(\tilde{f}-\tilde{g}) d x \\
& =\frac{1}{2} \int_{\Omega}\left|\frac{\partial}{\partial y} w\left(x^{\prime}, y\right)\right|\left(\int_{2 t-y}^{y}\left(\tilde{f}\left(x^{\prime}, \tau\right)-\tilde{g}\left(x^{\prime}, \tau\right)\right) d \tau\right) d x^{\prime} d y<0 .
\end{aligned}
$$

Since $j^{\prime}$ is nondecreasing and $u=u^{t}$, we have $j^{\prime}(u)=\left(j^{\prime}(u)\right)^{t}$ (see equation (3.6) in $[14])$, and in view of (11.26) it follows that $j^{\prime}(u) \neq 0$. Therefore we may take $h=j^{\prime}(u)$ in the equation above. Because of the convexity of $j$ we get then

$$
\int_{\Omega}(j(u)-j(v)) d x \leq \int_{\Omega} j^{\prime}(u)(u-v) d x<0,
$$


a contradiction. The theorem is proved.

Next we prove that solutions of the above considered problems are continuous from the left with respect to the parameter of symmetrization $t$.

Theorem 11.4. Let $(\cdot)^{t}, 0 \leq t \leq 1$, denote the continuous $(k, n)$-Steiner symmetrization into some $(k, n)$-Steiner symmetrization. Let $\Omega, f, c, \gamma$ be defined as in Theorem 11.2 and let $t_{m}, m=1,2, \ldots$ be an increasing sequence in the standard interval $I=[0,1]$ such that $t_{m} \rightarrow t_{0} \in I$. Further, let $v$ and $v_{m}$ be the positive minimal solutions to the problems $\mathbb{B}_{2}\left(\Omega^{t_{0}}, c, \gamma, f^{t_{0}}\right)$ and $\mathbb{B}_{2}\left(\Omega^{t_{m}}, c, \gamma, f^{t_{m}}\right)$, respectively. Then

$$
v_{m} \longrightarrow v \quad \text { in } W^{1,2}\left(\mathbb{R}^{n}\right) \text {. }
$$

Proof. (a) First we prove the theorem for the SC 1-symmetrization. Then $-\infty<t<$ $\infty$. Let $t_{m}$ be an increasing sequence such that $t_{m} \rightarrow t_{0} \in \mathbb{R}$. Since $\left(f^{t_{m}}\right)^{t_{0}}=f^{t_{0}}$ for $m=1,2, \ldots$, we may apply Theorem 10.4 to conclude that the functions $v_{m}$, $m=1,2, \ldots$ are equibounded in $W^{1,2}\left(\mathbb{R}^{n}\right)$. Therefore we can find a subsequence $v_{m^{\prime}}$ and a function $w \in W^{1,2}\left(\mathbb{R}^{n}\right)$, such that

$$
v_{m^{\prime}} \rightarrow w \quad \text { weakly in } W^{1,2}\left(\mathbb{R}^{n}\right)
$$

and

$$
v_{m^{\prime}} \longrightarrow w \quad \text { in } L^{2}\left(\mathbb{R}^{n}\right) \text { and a.e. }
$$

By Lemma 5.2, we have $\partial \Omega^{t_{0}} \subset \partial \Omega^{t_{m}}+r_{m} \bar{B}_{1}$, where $r_{m}=t_{0}-t_{m}$. Then $r_{m+1} \leq r_{m}$ for $m=1,2, \ldots$ and $r_{m} \rightarrow 0$. Since $v_{m} \equiv 0$ in $\mathbb{R}^{n} \backslash \Omega^{t_{m}}$, we conclude that $w \in W_{0}^{1,2}\left(\Omega^{t_{0}}\right)$. Thus we can argue as in the proof of Theorem 11.2 to derive that $w=v$.

Next since $\left(f^{t_{m}}\right)^{t_{m+1}}=f^{t_{m+1}}$ we obtain from Theorem 11.2 that

$$
v_{1} \prec^{t_{2}} v_{2} \prec^{t_{3}} \ldots \prec^{t_{0}} v \text {. }
$$

Together with (11.32) and the equation (11.25) of Corollary 11.3 this implies that the sequence of norms $\left\|v_{m}\right\|_{2}^{2}$ decreases and

$$
\left\|v_{m}\right\|_{2}^{2} \rightarrow\|v\|_{2}^{2} .
$$

By the uniform convexity of $L^{2}\left(\mathbb{R}^{n}\right)$ this means that

$$
v_{m} \longrightarrow v \quad \text { in } L^{2}\left(\mathbb{R}^{n}\right) \text {. }
$$

Now (11.34), combined with the fact that the functions $v_{m}, m=1,2, \ldots$ are equibounded in $W^{1,2}\left(\mathbb{R}^{n}\right)$, implies that

$$
v_{m} \rightarrow v \quad \text { weakly in } W^{1,2}\left(\mathbb{R}^{n}\right) .
$$

Finally we have,

$$
\left\|\nabla v_{m}\right\|_{2}^{2}+c\left\|v_{m}\right\|_{2}^{2}=\int_{\Omega^{t_{m}}}\left(\gamma\left(v_{m}\right)+f\right) v_{m} d x \rightarrow \int_{\Omega^{t_{0}}}(\gamma(v)+f) v d x=\|\nabla v\|_{2}^{2}+c\|v\|_{2}^{2} .
$$

In view of the uniform convexity of $W^{1,2}\left(\mathbb{R}^{n}\right)$ this yields (11.30).

(b) Now we prove the theorem for the continuous $(k, n)$-Steiner symmetrization and $t_{0}=1$. In this case, $\Omega^{t_{0}}=\Omega^{*}$ and $f^{t_{0}}=f^{*}$; i.e. $\Omega^{t_{0}}$ and $f^{t_{0}}$ are just the corresponding $(k, n)$-Steiner symmetrizations of $\Omega$ and $f$, respectively. Similarly, we have $\left(\Omega^{t_{m}}\right)^{*}=\Omega^{*}$ and $\left(f^{t_{m}}\right)^{*}=f^{*}$ for $m=1,2, \ldots$ Applying Theorem 10.1 [14], we conclude as before that the functions $v_{m}, m=1,2, \ldots$ are equibounded in $W^{1,2}\left(\mathbb{R}^{n}\right)$. Therefore we can find a subsequence $v_{m^{\prime}}$ and a function $w \in W^{1,2}\left(\mathbb{R}^{n}\right)$, such that (11.31) and (11.32) 
remain valid. By equation (9.5) of Theorem 9.2, we have $\partial \Omega^{t_{0}} \subset \partial \Omega^{t_{m}}+\varepsilon_{m} \bar{B}_{1}$ with some $\varepsilon_{m}>0$ such that $\varepsilon_{m} \rightarrow 0$ as $m \rightarrow \infty$. Since $v_{m} \equiv 0$ in $\mathbb{R}^{n} \backslash \Omega^{t_{m}}$, we conclude that $w \in W_{0}^{1,2}\left(\Omega^{t_{0}}\right)$. As in part (a), this implies that $w \equiv v$.

According to Definition 9.1, $\Omega^{t_{m+1}}$ and $f^{t_{m+1}}$ are obtained from $\Omega^{t_{m}}$ and $f^{t_{m}}$, respectively, after a finite number of $(k-1, n)$-Steiner symmetrizations followed by an appropriate SC $(k-1, n)$-Steiner symmetrization. Therefore, it follows from Theorem 10.1 in [14] and Theorem 11.2 of this section that the relations (11.33) remain valid in this case as well. As in part (a) of this proof, the latter yields (11.30).

(c) The theorem is proved for the $\mathrm{SC}$ one dimensional symmetrization and for the case $t_{0}=1$, which corresponds to the $(k, n)$-Steiner symmetrization. Now, the general case follows from these two cases via the standard inductive argument.

Since the proofs of Theorems 11.2 and 11.4 depend only on the maximum principle, we can derive similar results for parabolic problems. The proofs of Theorems 11.5 and 11.6 below are based on the approximation scheme involving solutions of some elliptic problems. This idea was used in [5] for the Schwarz symmetrization and then in [14] for the $(k, n)$-Steiner symmetrization. As we will see this method works also for continuous symmetrizations. First, following [14], we will define solutions of parabolic problems.

Definition 11.4. Let $\Omega \subset \mathbb{R}^{n}$ be a bounded open set, $c \geq 0, T>0, f \in L_{+}^{2}(\Omega \times(0, T))$, $\varphi \in L_{+}^{2}(\Omega)$, and let $\gamma: \mathbb{R}_{0}^{+} \rightarrow \mathbb{R}_{0}^{+}$be a globally Lipschitzian function. We say that $u$ solves the problem $\mathbb{I}(\Omega, T, c, \gamma, f, \varphi)$ if $u$ is a solution of the following initial boundary value problem:

$$
\begin{aligned}
& u \in L^{2}\left(0, T ; W_{0}^{1,2}(\Omega)\right) \cap C\left([0, T] ; L^{2}(\Omega)\right), \quad \frac{\partial u}{\partial t} \in L_{2}\left([0, T] ; L^{2}(\Omega)\right), \\
& u_{t}-\Delta u+c u=\gamma(u)+f \quad \text { in } \Omega \times(0, T), \\
& u(x, 0)=\varphi(x) \quad \text { in } \Omega .
\end{aligned}
$$

Under the assumptions of Definition 11.4 the problem $\mathbb{I}(\Omega, T, c, \gamma, f, \varphi)$ has a unique nonnegative solution that can be approximated by the so-called method of discretization in time, see [19] or [14, Section 10]. To define this approximation, we choose $N \in \mathbb{N}$. Then we divide the interval $(0, T)$ into $N$ subintervals $\left[t_{i-1}, t_{i}\right]$, where $t_{i}=i T / N$, and we set

$$
f_{i}(x)=\frac{T}{N} \int_{t_{i-1}}^{t_{i}} f(x, s) d s, \quad i=1, \ldots, N .
$$

We put $u_{0}=\varphi$. Then let $u_{i}$ be the solution to the problem

$$
\mathbb{B}_{1}\left(\Omega, c+(N / T), \gamma\left(u_{i-1}\right)+f_{i}+(N / T) u_{i-1}\right)
$$

defined inductively for all $i=1, \ldots, N$.

Let $u^{N}(x, t)$ denote the function of $x \in \Omega$ and $t \in[0, T]$ defined for $t_{i-1} \leq t \leq t_{i}$, $i=1, \ldots, N$ by

$$
u^{N}(x, t)=u_{i-1}(x)+\left(t-t_{i-1}\right)(N / T)\left(u_{i}(x)-u_{i-1}(x)\right) .
$$

The latter equation gives the desired approximation to the solution of the problem (11.35). Namely we have (see [19, Theorem 2.2.4,p.42 ff.]):

$$
u^{N}(\cdot, t) \rightarrow u(\cdot, t) \quad \text { weakly in } W_{0}^{1,2}(\Omega) \text { for all } t \in(0, T),
$$




$$
\frac{\partial u^{N}}{\partial t} \rightarrow \frac{\partial u}{\partial t} \quad \text { weakly in } L^{2}\left([0, T] ; L^{2}(\Omega)\right)
$$

and

$$
u^{N} \rightarrow u \quad \text { in } C\left(0, T ; L^{2}(\Omega)\right) .
$$

Theorem 11.5. Let $(\cdot)^{s}, 0 \leq s \leq 1$, denote a continuous $(k, n)$-Steiner symmetrization. Let $\Omega, c, T, f, \gamma, \varphi$ and $u$ be as in Definition 11.4 and let $g \in L_{+}^{2}\left(\Omega^{s} \times(0, T)\right)$, $\psi \in L_{+}^{2}\left(\Omega^{s}\right)$ with $f(\cdot, t) \prec^{s} g(\cdot, t)$ and $g(\cdot, t)=(g(\cdot, t))^{s}$ for all $t \in(0, T)$, and $\varphi \prec^{t} \psi$, $\psi=\psi^{t}$. Let $v$ be the solution of the problem $\mathbb{I}\left(\Omega^{t}, T, c, \gamma, g, \psi\right)$. Then

$$
u(\cdot, t) \prec^{s} v(\cdot, t) \quad \text { for all } t \in(0, T)
$$

and

$$
v(\cdot, t)=(v(\cdot, t))^{s} \quad \text { for all } t \in(0, T) .
$$

Proof. Fix $0<s \leq 1$. For any $N \in \mathbb{N}$ and $1 \leq i \leq N$, let $u_{i}$ be the solution to the problem (11.37) and let $v_{i}$ be the solution to the problem (11.37) with $\Omega, f$, and $\varphi$ replaced by $\Omega^{s}, g$, and $\psi$, respectively. By the assumptions we have $u_{0} \prec^{s} v_{0}$. If $N$ is large enough then $\gamma(\tau)+N \tau / T$ is increasing and convex in $\tau$. Since $f \prec^{s} g$, by (11.36) we also have $f_{i} \prec^{s} g_{i}$ for $i=1, \ldots, N$.

Applying Theorem 11.2, we conclude that $u_{i} \prec^{s} v_{i}$ for $i=1, \ldots, N$. By (11.38) it follows that $u^{N} \prec^{s} v^{N}$. Then passing to the limit as $N \rightarrow \infty$ we obtain (11.39).

Since $v_{i}(\cdot, t)=\left(v_{i}(\cdot, t)\right)^{s}$ for all $t \in(0, T)$ and all $i=1, \ldots, N$ we have $v^{N}(\cdot, t)=$ $\left(v^{N}(\cdot, t)\right)^{s}$. Passing to the limit as $N \rightarrow \infty$, this gives (11.40).

The solutions of the above parabolic problems are continuous from the left with respect to the parameter of symmetrization $s$.

Theorem 11.6. Let $\Omega, c, T, f, \gamma, \varphi$, and $u$ be as in Theorem 11.5 and let $s_{m}$, $m=1,2, \ldots$ be an increasing sequence in $I=[0,1]$ such that $s_{m} \rightarrow s_{0} \in I$. Further, let $v$ and $v_{m}$ be the positive solutions of the problems $\mathbb{I}\left(\Omega^{s_{0}}, T, c, \gamma, g^{s_{0}}, \psi\right)$ and $\left.\mathbb{I}\left(\Omega^{s_{m}}, T, c, \gamma, g^{s_{m}}, \psi\right)\right)$, respectively. Then

$$
v_{m} \longrightarrow v \quad \text { in } W^{1,2}\left(\mathbb{R}^{n}\right) \times(0, T) .
$$

Proof. Using discretization in time as in the proof of Theorem 11.5, we approximate $v$ and $v_{m}, m=1,2, \ldots$ with solutions $v^{(i)}$ and $v_{m}^{(i)}$ to the problem (11.37) for an appropriate initial data. By Theorem 11.4, $v_{m}^{(i)} \rightarrow v^{(i)}$ in $W^{1,2}\left(\mathbb{R}^{n}\right)$. Taking the limit as $N \rightarrow \infty$ and using Theorem 11.5, we obtain (11.41).

Remark 11.1. The major results in Sections 10 and 11 remain valid if we replace the operator $(-\Delta+c)$ by any uniformly elliptic operator which is invariant under considered transformations. In the case of the continuous $k, n)$-Steiner symmetrization this is true for instance for operators of the type

$$
-\sum_{i=1}^{n-k} \frac{\partial}{\partial x_{i}}\left(\sum_{j=1}^{n-k} a_{i j}\left(x^{\prime}\right) \frac{\partial}{\partial x_{j}}+b_{i}\left(x^{\prime}\right)\right)-\sum_{i=n-k+1}^{n} \frac{\partial^{2}}{\partial x_{i}^{2}}+c\left(x^{\prime}\right),
$$

where the coefficients $a_{i j}, b_{i}$ and $c$ are bounded and independent of $y=\left(x_{n-k+1}, \ldots, x_{n}\right)$, $c$ is nonnegative and

$$
\sum_{i, j=1}^{n-k} a_{i j}\left(x^{\prime}\right) \xi_{i} \xi_{j} \geq \lambda \sum_{i=1}^{n-k} \xi_{i}^{2}, \quad \lambda>0
$$




\section{Appendix}

In this appendix we prove Theorem 3.1. For compact sets $\Omega$ and even $j=2 s$, equation (3.9) is a part of Theorem 4.32 in [25]. If $j=2 s-1$ is odd, we apply an even number of symmetrizations to the set $\Omega_{1}$ and again obtain (3.9).

To prove (3.11) for $\Omega \in \mathcal{F}_{n}$, we consider a non-empty slice $\Omega\left(x^{\prime}\right)$ that is compact in $\mathbb{R}^{k}$. Applying (3.9) to symmetrizations in slices we obtain

$$
\lim _{j \rightarrow \infty} d\left(\Omega_{j}\left(x^{\prime}\right), \Omega^{*}\left(x^{\prime}\right)\right)=0 .
$$

Since $\Omega^{*}\left(x^{\prime}\right)$ is a $k$-dimensional ball and $\mathcal{L}^{k}\left(\Omega_{j}\left(x^{\prime}\right)\right)=\mathcal{L}^{k}\left(\Omega^{*}\left(x^{\prime}\right)\right)$, equation (12.1) implies (3.11) in the case of compact sets.

In the rest of this section, we work with open bounded sets $\Omega$. Proving (3.10), we may assume without loss of generality that all open sets under consideration belong to the unit ball $B^{(n)}$.

First we prove three technical lemmas. In all these lemmas, by $S$ we denote the $(k, k)$ Steiner symmetrization in $\mathbb{R}^{k}$ with respect to the origin $x=(0, \ldots, 0)$. Then let $S_{1}$ and $S_{2}$ be $(k-1, k)$-Steiner symmetrizations, which approximate $S$ in the sense of Theorem 3.1, and let $\Sigma_{1}$ and $\Sigma_{2}$ be the symmetry planes (one dimensional) of the symmetrizations $S_{1}$ and $S_{2}$, respectively. For notational convenience we will assume without loss of generality that $\Sigma_{1}=\left\{x=(t \cos \gamma \pi, t \sin \gamma \pi, 0, \ldots, 0) \in \mathbb{R}^{k}:-\infty<t<\right.$ $\infty\}$ for some irrational $\gamma \in(0,1 / 2)$ and $\Sigma_{2}$ is the $x_{1}$-axis.

For given $R, x_{1}$, and $\rho$ such that $R>0,-R \leq x_{1} \leq R$, and $0<\rho<R$, let

$$
Z\left(x_{1}, R, \rho\right)=\overline{B_{R}^{(k)}} \cap\left(\cup_{\zeta} B_{\rho}^{(k)}(\zeta)\right),
$$

where the union is taken over all balls $B_{\rho}^{(k-1)}(\zeta)$ centered at $\zeta=\left(\zeta_{1}, \ldots, \zeta_{k}\right) \in \mathbb{R}^{k}$ such that $|\zeta|=R$ and $\zeta_{1}=x_{1}$.

Lemma 12.1. Let $0<r<R_{1}<1$. Then there exists $\tau=\tau\left(r, R_{1}\right), 0<\tau<1$, such that for every compact set $K$ satisfying the conditions $K \subset \overline{B_{R}^{(k)}}$ for some $R \in\left[R_{1}, 1\right]$ and $\mathcal{L}^{k}(K) \leq \mathcal{L}^{k}\left(B_{r}^{(k)}\right)$ there is real $x_{1}=x_{1}(K)$ such that $-r \leq x_{1} \leq r$ and

$$
\overline{B_{R}^{(k)}} \backslash K_{2} \supset Z\left(x_{1}, R, \tau R\right) .
$$

Proof. Since $K_{2}$ is Steiner symmetric with respect to $\Sigma_{2}$ and since $\mathcal{L}^{k}\left(K_{2}\right) \leq \mathcal{L}^{k}\left(B_{r}^{(k)}\right)$ there is a point $x_{0}=\left(x_{1}^{0}, x_{2}^{0}, 0, \ldots, 0\right) \in S_{r}^{(k)} \backslash K_{2}$ such that $-r \leq x_{1}^{0} \leq r$ and $x_{2}^{0}=$ $\sqrt{r^{2}-\left(x_{1}^{0}\right)^{2}} \geq 0$.

Since the slice $K_{2}\left(x_{1}^{0}\right)$ is a $(k-1)$-dimensional ball we have $K_{2}\left(x_{1}^{0}\right) \subset B_{x_{2}^{0}}^{(k-1)}$. Since at the same time $K_{2}\left(x_{1}^{0}\right)$ is the result of $S_{2}$ symmetrization of $K_{1}\left(x_{1}^{0}\right)$ we have

$$
\begin{aligned}
\mathcal{L}^{k-1}\left(B_{R}^{(k)}\left(x_{1}^{0}\right) \backslash K_{1}\right) & =\mathcal{L}^{k-1}\left(B_{R}^{(k)}\left(x_{1}^{0}\right) \backslash K_{2}\right) \\
& \geq \mathcal{L}^{k-1}\left(B_{R_{1}}^{(k)}\left(x_{1}^{0}\right) \backslash B_{x_{2}^{0}}^{(k-1)}\right) \geq c\left(r, R_{1}\right),
\end{aligned}
$$

where

$$
c\left(r, R_{1}\right)=\min _{\left|x_{1}^{0}\right| \leq r} \mathcal{L}^{k-1}\left(B_{R_{1}}^{(k)}\left(x_{1}^{0}\right) \backslash B_{x_{2}^{0}}^{(k-1)}\right)>0 .
$$


For $x \in B_{R}^{(k)} \backslash \Sigma_{1}$, let $\Gamma_{1}(x)$ denote the ray through $x$ that is orthogonal to $\Sigma_{1}$ and has its origin at some point $\tilde{x} \in \Sigma_{1} \cap B_{R}^{(k)}$. Let $I(x)$ be a closed segment of $\Gamma_{1}(x)$ joining $x$ and $\partial B_{R}^{(k)}$. We note that $I(x) \cap K_{1}=\emptyset$ if $x \notin K_{1}$. Let

$$
J\left(x_{1}^{0}\right)=\cup_{x} I(x),
$$

where the union is taken over all $x \in B_{R}^{(k)}\left(x_{1}^{0}\right) \backslash K_{1}\left(x_{1}^{0}\right)$. Let $A=A\left(K, x_{1}^{0}\right)$ be the maximal segment of the $x_{1}$-axis such that $x_{1}^{0} \in A$ and if $x_{1} \in A$ then

$$
\mathcal{L}^{k-1}\left(\mathbb{R}^{k}\left(x_{1}\right) \cap J\left(x_{1}^{0}\right)\right) \geq(1 / 2) c\left(r, R_{1}\right) .
$$

Using (12.2), one can show that there is a constant $t=t\left(r, R_{1}\right)>0$ such that

$$
\mathcal{L}^{1}\left(A\left(K, x_{1}^{0}\right)\right) \geq t\left(r, R_{1}\right)
$$

for every compact set $K$ satisfying the assumptions of the lemma.

Finally, it is not difficult to see that (12.3) and (12.4) imply the lemma.

The following lemma allows us to control how fast the approximation process is for compact sets.

Lemma 12.2. Let $0<r<R_{1}<1$. There exist a positive integer $N=N\left(r, R_{1}\right)$ and real $\beta=\beta\left(r, R_{1}\right), 0<\beta<1$, such that for all $j \geq N$ and all $R$ such that $R_{1} \leq R \leq 1$, we have

$$
K_{j} \subset \overline{B_{\beta R}^{(k)}}
$$

for every compact set $K \subset \overline{B_{R}^{(k)}}$ such that $\mathcal{L}^{k}(K) \leq \mathcal{L}^{k}\left(B_{r}^{(k)}\right)$.

Proof. We may assume without loss of generality that

$$
K \subset \overline{B_{R}^{(k)}} \backslash Z\left(x_{1}, R, \tau R\right)
$$

with $\tau=\tau\left(r, R_{1}\right)>0$ defined in Lemma 12.1 and some $x_{1}=x_{1}(K)$ such that $-r \leq$ $x_{1} \leq r$. Indeed, if $K$ does not satisfy (12.5), then we replace $K$ with its second symmetrization $K_{2}$, which by Lemma 12.1 satisfies the required condition.

Let $C_{R}$ be the circle in the plane $\mathcal{E}=\left\{x \in \mathbb{R}^{k}: x=\left(x_{1}, x_{2}, 0, \ldots, 0\right)\right\}$. Using complex notation, we will write $R e^{i \theta}$ for $x=(R \cos \theta, R \sin \theta, 0, \ldots, 0) \in C_{R}$.

It follows from (12.5) that there is a sufficiently small constant $a_{0}=a_{0}\left(r, R_{1}\right)>0 \mathrm{such}$ that for every compact set $K$ satisfying the conditions of the lemma there is a point $R e^{i \theta_{0}}$ with $\theta_{0}=\theta_{0}(K)$ such that the intersection $C_{R} \cap Z\left(x_{1}, R, \tau R\right)$ contains an arc $\alpha$ centered at $R e^{i \theta_{0}}$ with the angular measure $\geq a_{0}$.

In addition, it is not difficult to see that there exists a constant $\nu=\nu\left(r, R_{1}\right), 0<\nu<1$, such that the set $C_{R} \cap Z\left(x_{1}, R, \nu \tau R\right)$ contains a subarc $\alpha^{\prime} \subset \alpha$ again centered at $R e^{i \theta_{0}}$, which has the angular measure $\geq(2 / 3) a_{0}$.

Let $\operatorname{Ref}_{1}$ and $\operatorname{Ref}_{2}$ denote the reflections in the plane $\mathcal{E}$ with respect to the lines $l_{1}=\left\{z=t e^{i \gamma \pi}:-\infty<t<\infty\right\}$ and $l_{2}=\{z=t:-\infty<t<\infty\}$, respectively. Let $\mathcal{R}^{s}=\left(\operatorname{Ref}_{1} \circ \operatorname{Ref}_{2}\right)^{s}, s=2,3, \ldots$ Then $\mathcal{R}^{s}\left(\operatorname{Re}^{i \theta_{0}}\right)=\operatorname{Re}^{i\left(2 s \gamma \pi+\theta_{0}\right)}, s=2,3, \ldots$.

Since $\gamma$ is irrational, the set $\left\{R e^{2 i s \gamma \pi}\right\}_{s=2}^{\infty}$ is dense in $C_{R}$; see, for example, [25, Lemma 3.25]. Therefore there are indices $s_{1}, \ldots, s_{N_{1}}$ such that for any $\theta_{0}$, the points $R e^{i\left(2 s_{j} \gamma \pi+\theta_{0}\right)}, j=1, \ldots, N_{1}$, divide $C_{R}$ into $N_{1}$ arcs, each of which has the angular measure $\leq a_{0} / 4$. Let $N=\max \left\{s_{1}, \ldots, s_{N_{1}}\right\}$. Let

$$
\alpha_{s}=\mathcal{R}^{s}(\alpha), \quad \alpha_{s}^{\prime}=\mathcal{R}^{s}\left(\alpha^{\prime}\right), \quad s=2,3, \ldots, N,
$$


where $\alpha$ and $\alpha^{\prime}$ are the arcs defined above. By our choice of the points $R e^{i\left(2 s_{j} \gamma \pi+\theta_{0}\right)}$, we have

$$
C_{R}=\cup_{s=1}^{N} \alpha_{s}^{\prime}=\cup_{s=1}^{N} \alpha_{s} .
$$

Let

$$
W\left(x_{1}\right)=\overline{B_{R}^{(k)}} \backslash Z\left(x_{1}, R, \tau R\right), \quad W^{\prime}\left(x_{1}\right)=\overline{B_{R}^{(k)}} \backslash Z\left(x_{1}, R, \nu \tau R\right),
$$

where $x_{1}=x_{1}(K)$ and $\tau$ and $\nu$ are positive constants defined in the beginning of this proof. For $s=1,2, \ldots$ and $x_{1}=x_{1}(K)$, let

$$
W_{s}\left(x_{1}\right)=\left(S_{2} \circ S_{1}\right)^{s}\left(W\left(x_{1}\right)\right), \quad W_{s}^{\prime}\left(x_{1}\right)=\left(S_{2} \circ S_{1}\right)^{s}\left(W^{\prime}\left(x_{1}\right)\right) .
$$

It follows from (12.5) and our construction that

$$
K_{2 N} \subset W_{N}\left(x_{1}\right) \subset W_{N}^{\prime}\left(x_{1}\right)
$$

and

$$
W_{N}^{\prime}\left(x_{1}\right) \cap S_{R}^{(k)}=\emptyset
$$

Let

$$
\beta\left(x_{1}\right)=\inf \left\{\beta>0: W_{N}\left(x_{1}\right) \subset \overline{B^{(k)}} \beta R\right\} .
$$

By (12.6), $0<\beta\left(x_{1}\right)<1$ for all $x_{1}$ such that $-r \leq x_{1} \leq r$. Let $\tilde{\beta}=\sup _{\left|x_{1}\right| \leq r} \beta\left(x_{1}\right)$. Our construction above works for every compact set $K$ satisfying the assumptions of the lemma. Choosing $K=\overline{B_{r}^{(k)}}$, we obtain that $\tilde{\beta} \geq r / R \geq r>0$.

Thus, to complete the proof, we have to show that $\tilde{\beta}<1$. If not, we can find a sequence $x_{1}^{(s)} \in[-r, r], s=1,2, \ldots$, such that $x_{1}^{(s)} \rightarrow x_{1}^{(0)}$ and $\beta\left(x_{1}^{(s)}\right) \rightarrow 1$ as $s \rightarrow \infty$. For all sufficiently large $s$ we have

$$
Z\left(x_{1}^{(0)}, R, \nu \tau R\right) \subset Z\left(x_{1}^{(s)}, R, \tau R\right) .
$$

Therefore for all such $s$ we have

$$
W_{N}^{\prime}\left(x_{1}^{(0)}\right) \supset W_{N}\left(x_{1}^{(s)}\right) .
$$

By $(12.6), W_{N}^{\prime}\left(x_{1}^{(0)}\right) \subset \overline{B_{\beta^{\prime} R}^{(k)}}$ for some $0<\beta^{\prime}<1$. Hence for all sufficiently large $s$ we have

$$
W_{N}\left(x_{1}^{(s)}\right) \subset \overline{B_{\beta^{\prime} R}^{(k)}}
$$

contradicting the assumption that $\beta\left(x^{(s)}\right) \rightarrow 1$ as $s \rightarrow \infty$. The proof of the lemma is complete.

Lemma 12.3. Let $S, S_{1}$, and $S_{2}$ be the symmetrizations as in Lemmas 12.1 and 12.2. Let $0<r<R$ and $0<\rho<R$. Then there is a constant $c_{1}=c_{1}(r, \rho, R)>0$ such that

$$
\mathcal{L}^{k}\left(\Omega_{1} \backslash B_{r}^{(k)}\right) \geq c_{1}
$$

for every open set $\Omega \subset \mathbb{R}^{k}$ such that $\Omega_{2}$ contains some point $x_{0}=\left(x_{0}^{\prime}, y_{0}\right)$ with $x_{0}^{\prime} \in \mathbb{R}$, $y_{0} \in \mathbb{R}^{k-1}$ such that $\left|x_{0}\right| \geq R$ and $\left|y_{0}\right| \geq \rho$.

Proof. Let $\Omega$ satisfies the assumptions of the lemma and let

$$
c_{2}=c_{2}(r, \rho, R)=\min _{x_{0}^{\prime}} \mathcal{L}^{k-1}\left(\left(\left\{x_{0}^{\prime}\right\} \times B_{\rho}^{(k-1)}\right) \backslash B_{r}^{(k)}\right),
$$

where the minimum is taken over all $x_{0}^{\prime}$ such that $\left(x_{0}^{\prime}\right)^{2}+\rho^{2} \geq R^{2}$. It is clear that $c_{2}>0$. Since $\Omega_{2}\left(x_{0}^{\prime}\right)$ is a $(k-1)$-dimensional ball with the radius $\geq \rho$, we have $\mathcal{L}^{k-1}\left(\Omega_{2}\left(x_{0}^{\prime}\right) \backslash B_{r}^{(k)}\right) \geq c_{2}$. 
For $x \notin \Sigma_{1}$, let $\hat{I}(x)$ denote the segment orthogonal to $\Sigma_{1}$ that joins $x$ and $\Sigma_{1}$. For $x \in \mathbb{R}^{k} \backslash\left(B_{r}^{(k)} \cup \Sigma_{1}\right)$, we set $I(x)=\hat{I}(x) \backslash B_{r}^{(k)}$. We note that $I(x) \subset \Omega_{1}$, whenever $x \in \Omega_{1}$. For $x_{0}^{\prime} \in \mathbb{R}$ defined above, let

$$
J_{1}\left(x_{0}^{\prime}\right)=\cup_{x} I(x),
$$

where the union is taken over all $x=\left(x_{0}^{\prime}, y\right) \in \mathbb{R}^{k} \backslash B_{r}^{(k)}$ such that $y \in \Omega_{1}\left(x_{0}^{\prime}\right)$. Let $A_{1}\left(\Omega, x_{0}^{\prime}\right)$ be the maximal closed segment of the $x_{1}$-axis such that $x_{0}^{\prime} \in A_{1}\left(\Omega, x_{0}^{\prime}\right)$ and

$$
\mathcal{L}^{k-1}\left(\left(J_{1}\left(x_{0}^{\prime}\right)\right)\left(x_{1}\right)\right) \geq c_{2} / 2,
$$

where $\left(J_{1}\left(x_{0}^{\prime}\right)\right)\left(x_{1}\right)$ denotes the $(k-1)$-slice of $J_{1}\left(x_{0}^{\prime}\right)$ at $x_{1}$. It is not difficult to see that there is a constant $t_{1}=t_{1}(r, \rho, R)>0$ such that

$$
\mathcal{L}^{1}\left(A_{1}\left(\Omega, x_{0}^{\prime}\right)\right) \geq t_{1}
$$

for every domain $\Omega$ satisfying the assumptions of the lemma.

Since $J_{1}\left(x_{0}^{\prime}\right) \subset \Omega_{1}$, equations (12.8) and (12.9) imply (12.7).

Proof of Theorem 3.1 for open sets. The proof is by contradiction. Suppose that equation (3.10) does not hold for some open set $\Omega \subset B^{(k)}$. Then we have to consider the following two cases.

(i) There are real number $\varepsilon_{0}>0$ and a sequence $x_{s} \rightarrow x_{0}$ such that $x_{s} \in \partial \Omega_{m_{s}}$ for some subsequence of indices $m_{s}$ and

$$
d\left(x_{s}, \partial \Omega^{*}\right) \geq \varepsilon_{0} \quad \text { for } s=0,1, \ldots
$$

(ii) There are real number $\varepsilon_{0}>0$ and a sequence $x_{s} \rightarrow x_{0}$ such that $x_{s} \in \partial \Omega^{*}$ for all $s$ and there is a subsequence of indices $m_{s}$ such that

$$
d\left(x_{s}, \partial \Omega_{m_{s}}\right) \geq \varepsilon_{0} \quad \text { for } s=1,2, \ldots
$$

In each of these cases we may assume without loss of generality that all indices $m_{s}$ are odd and $m_{s} \geq 3$. Then $\Omega_{m_{s}}=S_{1}\left(\Omega_{m_{s}-1}\right)$.

To prove (i), we consider two subcases.

(1) We first assume that $B_{\varepsilon_{0}}^{(n)}\left(x_{0}\right) \subset \Omega^{*}$. Let $x_{s}=\left(x_{s}^{\prime}, y_{s}\right), s=0,1, \ldots$ Then there are constants $\delta_{0}>0$ and $R>\left|y_{0}\right|$ such that

$$
\Omega^{*}\left(x^{\prime}\right) \supset B_{R}^{(k)} \text { for all } x^{\prime} \in B_{\delta_{0}}^{(n-k)}\left(x_{0}^{\prime}\right) .
$$

Next we estimate how much the slice $\Omega_{m_{s}-1}\left(x_{s}^{\prime}\right)$ differs from the ball $B_{R}^{(k)}$. Let $L_{1}\left(x^{\prime}, y\right)$ and $L_{2}\left(x^{\prime}, y\right)$ be the symmetrizing planes through $\left(x^{\prime}, y\right) \in \mathbb{R}^{n-k} \times \mathbb{R}^{k}$ of $S_{1}$ and $S_{2}$, respectively. Then $L_{j}\left(x^{\prime}, y\right)$ can be represented in the form $L_{j}\left(x^{\prime}, y\right)=\left\{\left(x^{\prime}, t\right): t \in\right.$ $\left.L_{j}(y)\right\}$, where $L_{j}(y)$ is a $(k-1)$-dimensional plane in $\mathbb{R}^{k}$, which does not depend on $x^{\prime}$.

Since $y_{s} \rightarrow y_{0}$ and $R>\left|y_{0}\right|$, we have that $\left|y_{s}\right|<R_{1}=\left(R+\left|y_{0}\right|\right) / 2$ for all sufficiently large $s$. Since $\Omega_{m_{s}}$ is the $(k-1)$-dimensional Steiner symmetrization of $\Omega_{m_{s}-1}$ with respect to $S_{1}$, the set $\Omega_{m_{s}}\left(x_{s}^{\prime}\right) \cap L_{1}\left(y_{s}\right)$ is a $(k-1)$-dimensional ball in the corresponding $(k-1)$-dimensional plane. Since $\left(x_{s}^{\prime}, y_{s}\right) \in \partial \Omega_{m_{s}}$, the latter implies that

$$
\Omega_{m_{s}}\left(x_{s}^{\prime}\right) \cap L_{1}\left(y_{s}\right) \subset B_{R_{1}}^{(k)} \cap L_{1}\left(y_{s}\right) \subset B_{R}^{(k)} \cap L_{1}\left(y_{s}\right) .
$$

This implies that there is $\delta_{1}=\delta_{1}\left(R,\left|y_{0}\right|\right)>0$ such that

$$
\mathcal{L}^{k-1}\left(\left(B_{R}^{(k)} \cap L_{1}\left(y_{s}\right)\right) \backslash\left(\Omega_{m_{s}}\left(x_{s}^{\prime}\right) \cap L_{1}\left(y_{s}\right)\right)\right) \geq \delta_{1}
$$


for all sufficiently large $s$. Since $\Omega_{m_{s}}=S_{1}\left(\Omega_{m_{s}-1}\right)$, the latter inequality implies that

$$
\mathcal{L}^{k-1}\left(\left(B_{R}^{(k)} \cap L_{1}\left(y_{s}\right)\right) \backslash\left(\Omega_{m_{s}-1}\left(x_{s}^{\prime}\right) \cap L_{1}\left(y_{s}\right)\right)\right) \geq \delta_{1}
$$

for all sufficiently large $s$.

Now since $\Omega_{m_{s}-1}\left(x_{s}^{\prime}\right)$ omits a set of positive $(k-1)$-dimensional measure in $B_{R}^{(k)} \cap L_{1}\left(y_{s}\right)$ and at the same time $\Omega_{m_{s}-1}\left(x_{s}^{\prime}\right) \cap L_{2}\left(y_{s}\right)$ is a $(k-1)$-dimensional ball in $L_{2}\left(y_{s}\right)$, we can argue as in the proof of Lemma 12.3 (cf. how (12.7) follows from (12.8) and (12.9)) to deduce that there is $\delta_{2}=\delta_{2}\left(R,\left|y_{0}\right|\right)>0$ such that

$$
\mathcal{L}^{k}\left(B_{R}^{(k)} \backslash \Omega_{m_{s}-1}\left(x_{s}^{\prime}\right)\right) \geq \delta_{2}
$$

for all sufficiently large $s$.

Next we show that (12.14) leads to a contradiction. The slice $\Omega\left(x_{0}^{\prime}\right)$ is an open set in $B^{(k)}$. Therefore for arbitrary small $\varepsilon>0$ there is a compact set $F \subset \Omega\left(x_{0}^{\prime}\right)$ such that

$$
\mathcal{L}^{k}\left(\Omega\left(x_{0}^{\prime}\right) \backslash F\right)<\varepsilon .
$$

Then for sufficiently small $\varepsilon_{1}>0$, we have $B_{\varepsilon_{1}}^{(n-k)}\left(x_{0}^{\prime}\right) \times F \subset \Omega$. By the monotonicity property of symmetrizations, we have

$$
\left(\left(\left\{x_{0}^{\prime}\right\} \times F\right)_{m_{s}-1}\right)\left(x_{0}^{\prime}\right) \subset \Omega_{m_{s}-1}\left(x_{s}^{\prime}\right),
$$

where $\left(\left(\left\{x_{0}^{\prime}\right\} \times F\right)_{m_{s}-1}\right)\left(x_{0}^{\prime}\right)$ denotes the slice of $\left(\left\{x_{0}^{\prime}\right\} \times F\right)_{m_{s}-1}$ at $x_{0}^{\prime}$. Applying equation (3.11) of Theorem 3.1 to the compact sets $\left\{x^{\prime}\right\} \times F$ and $\left\{x^{\prime}\right\} \times S(F)$, we obtain the following limit relation for measure in slices

$$
\mathcal{L}^{k}\left(\left(\left(\left(\left\{x^{\prime}\right\} \times F\right)_{j}\right)\left(x^{\prime}\right)\right) \triangle\left(\left(\left(\left\{x^{\prime}\right\} \times S(F)\right)\right)\left(x^{\prime}\right)\right)\right) \rightarrow 0 \quad \text { as } j \rightarrow \infty .
$$

Now, (12.12), (12.15), and (12.16) imply that

$$
\mathcal{L}^{k}\left(B_{R}^{(k)} \backslash\left(\left(\left\{x_{s}^{\prime}\right\} \times F\right)_{m_{s}-1}\right)\left(x_{s}^{\prime}\right)\right) \leq 2 \varepsilon
$$

for all sufficiently large $s$. Since $\left(\left(\left\{x_{s}^{\prime}\right\} \times F\right)_{m_{s}-1}\right)\left(x_{s}^{\prime}\right) \subset \Omega_{m_{s}-1}\left(x_{s}^{\prime}\right)$, the latter inequality contradicts (12.14). This completes the proof of the theorem in the case under consideration.

(2) In the second case, we assume that $B_{\varepsilon_{0}}^{(n)}\left(x_{0}\right) \cap \Omega^{*}=\emptyset$. We recall that $x_{s}=$ $\left(x_{s}^{\prime}, y_{s}\right) \rightarrow x_{0}=\left(x_{0}^{\prime}, y_{0}\right)$ as $s \rightarrow \infty$.

If $y_{0}=(0, \ldots, 0)$, then $\Omega^{*}\left(x^{\prime}\right)=\emptyset$ for all $x^{\prime} \in \mathbb{R}^{n-k}$ sufficiently close to $x_{0}^{\prime}$. Hence, $\Omega\left(x^{\prime}\right)=\emptyset$ and therefore $\Omega_{j}\left(x^{\prime}\right)=\emptyset$ for all such $x^{\prime}$ and all $j=1,2, \ldots$ It is easily seen that the latter contradicts our assumptions that $x_{s} \in \partial \Omega_{m_{s}}$ and $x_{s} \rightarrow x_{0}$ as $s \rightarrow \infty$.

Assume now that $\left|y_{0}\right|=R_{0}>0$. Since $B_{\varepsilon_{0}}^{(n)}\left(x_{0}\right) \cap \Omega^{*}=\emptyset$ there is $\delta_{0}>0$ such that for every $x^{\prime} \in \overline{B_{\delta_{0}}^{(n-k)}}\left(x_{0}^{\prime}\right)$ the slice $\Omega^{*}\left(x^{\prime}\right)$ is either an empty set or an open $k$-dimensional ball $B_{R\left(x^{\prime}\right)}^{(k)}$ with the radius $R\left(x^{\prime}\right)$ such that

$$
0<R\left(x^{\prime}\right) \leq \rho<R_{0}
$$

with some $\rho$ independent of $x^{\prime} \in \overline{B_{\delta_{0}}^{(n-k)}}\left(x_{0}^{\prime}\right)$. In particular, (12.17) shows that

$$
0<\mathcal{L}^{k}\left(\Omega\left(x^{\prime}\right)\right)=\mathcal{L}^{k}\left(\Omega^{*}\left(x^{\prime}\right)\right) \leq \mathcal{L}^{k}\left(B_{\rho}^{(k)}\right)
$$

for all $x^{\prime} \in \overline{B_{\delta_{0}}^{(n-k)}}\left(x_{0}^{\prime}\right)$. 
Let $\varepsilon_{0}>0$ be fixed and sufficiently small. For every $x^{\prime} \in \overline{B_{\delta_{0}}^{(n-k)}}\left(x_{0}^{\prime}\right)$, we choose a $k$-dimensional compact set $K\left(x^{\prime}\right)$ such that $K\left(x^{\prime}\right) \subset \Omega\left(x^{\prime}\right)$ and

$$
\mathcal{L}^{k}\left(\Omega\left(x^{\prime}\right) \backslash K\left(x^{\prime}\right)\right) \leq \varepsilon_{0} .
$$

By (12.18), we have

$$
\mathcal{L}^{k}\left(K\left(x^{\prime}\right)\right) \leq \mathcal{L}^{k}\left(B_{\rho}^{(k)}\right)
$$

for all $x^{\prime} \in \overline{B_{\delta_{0}}^{(n-k)}}\left(x_{0}^{\prime}\right)$.

Let $\varepsilon_{2}>0$ be sufficiently small and let $\varepsilon_{1}>0$ be such that $\rho+\varepsilon_{1}<R_{0}$ and

$$
\mathcal{L}^{k}\left(B_{\rho+\varepsilon_{1}}^{(k)} \backslash B_{\rho}^{(k)}\right)<\varepsilon_{2}
$$

for all $\rho \leq R_{0}$.

For $j=1,2, \ldots$ and $x^{\prime} \in \overline{B_{\delta_{0}}^{(n-k)}}\left(x_{0}^{\prime}\right)$, let $K^{j}\left(x^{\prime}\right)$ denote the slice at $x^{\prime}$ of the $j$-th successive symmetrization of the set $\left\{x^{\prime}\right\} \times K\left(x^{\prime}\right)$ defined by formulas (3.7) and (3.8). Alternatively, $K^{j}\left(x^{\prime}\right)$ can be obtained by applying appropriate $(k-1)$-dimensional symmetrizations to the set $K\left(x^{\prime}\right)$ in $\mathbb{R}^{k}$.

Since $K\left(x^{\prime}\right) \subset B^{(k)}$ for all $x^{\prime}$ and since $K\left(x^{\prime}\right)$ satisfies (12.20) for all $x^{\prime} \in \overline{B_{\delta_{0}}^{(n-k)}}\left(x_{0}^{\prime}\right)$, we can apply Lemma 12.2 with $r=\rho$ and $R_{1}=\rho+\varepsilon_{1}$ to the compact sets $K\left(x^{\prime}\right)$. This implies that there exists a positive integer $N=N\left(\rho, \varepsilon_{1}\right)$ such that

$$
K^{j}\left(x^{\prime}\right) \subset B_{\rho+\varepsilon_{1}}^{(k)}
$$

for all $x^{\prime} \in \overline{B_{\delta_{0}}^{(n-k)}}\left(x_{0}^{\prime}\right)$ and all $j \geq N$.

Now, combining (12.19), (12.21), and (12.22), we obtain that

$$
\mathcal{L}^{k}\left(\Omega_{j}\left(x^{\prime}\right) \backslash B_{\rho}^{(k)}\right) \leq \varepsilon_{0}+\varepsilon_{2}
$$

for all $x^{\prime} \in \overline{B_{\delta_{0}}^{(n-k)}}\left(x_{0}^{\prime}\right)$ and all $j \geq N$.

Now we return to the sequence $x_{s}=\left(x_{s}^{\prime}, y_{s}\right) \rightarrow x_{0}=\left(x_{0}^{\prime}, y_{0}\right)$. Since $x_{s} \in \partial \Omega_{m_{s}}$, for every $s$ we can find a point $\hat{x}_{s}=\left(\hat{x}_{s}^{\prime}, \hat{y}_{s}\right) \in \Omega_{m_{s}}$ such that $\hat{x}_{s} \rightarrow x_{0}$ as $s \rightarrow \infty$. Then, of course, $\Omega\left(\hat{x}_{s}^{\prime}\right) \neq \emptyset$ and therefore $\Omega^{*}\left(\hat{x}_{s}^{\prime}\right) \neq \emptyset$.

Suppose first that $x_{0} \notin \Sigma_{2}$. Then $d=d\left(x_{0}, \Sigma_{2}\right)>0$. Now we can apply Lemma 12.3 with $r=\rho, R=\left(R_{0}+\rho\right) / 2$, and $\rho=d$ and with the domains $\Omega_{1}$ and $\Omega_{2}$ in that lemma replaced by the domains $\Omega_{m_{s}-1}\left(\hat{x}_{s}^{\prime}\right)$ and $\Omega_{m_{s}}\left(\hat{x}_{s}^{\prime}\right)$, respectively. By Lemma 12.3 there exists a constant $c_{1}=c_{1}\left(\rho, d, R_{0}\right)>0$ such that

$$
\mathcal{L}^{k}\left(\Omega_{m_{s}-1}\left(x_{s}^{\prime}\right) \backslash B_{\rho}^{(k)}\right) \geq c_{1}
$$

for all sufficiently large $s$, which obviously contradicts (12.23) if $\varepsilon_{0}$ and $\varepsilon_{2}$ are chosen sufficiently small. This proves the theorem in the case under consideration if $x_{0} \notin \Sigma_{2}$.

Suppose now that $x_{0} \in \Sigma_{2}$. For every $s=1,2, \ldots$, the symmetrizing plane $L_{2}\left(\hat{y}_{s}\right)$ contains some point $\tilde{x}_{s}=\left(\hat{x}_{s}^{\prime}, \tilde{y}_{s}\right) \in \partial \Omega_{m_{s}-1}$. Selecting a subsequence if necessary, we may assume that $\tilde{x}_{s} \rightarrow \tilde{x}^{(1)}=\left(x_{0}^{\prime}, \tilde{y}^{(1)}\right)$.

Since $B_{\varepsilon_{0}}^{(n)}\left(x_{0}\right) \cap \Omega^{*}=\emptyset$ and $x_{0} \in \Sigma_{2}$, it follows from the definition of $(k, n)$-Steiner symmetrization that

$$
B_{\varepsilon_{0}}^{(n)}\left(\tilde{x}^{(1)}\right) \cap \Omega^{*}=\emptyset .
$$


Now, if $\tilde{x}^{(1)} \notin \Sigma_{1}$, to complete the proof, we can apply our argument above replacing the plane $\Sigma_{2}$ and the sequence of points $x_{s} \in \partial \Omega_{m_{s}}$ with the plane $\Sigma_{1}$ and the sequence $\tilde{x}_{s} \in \partial \Omega_{m_{s}-1}$, respectively.

In the case $\tilde{x}^{(1)} \in \Sigma_{1}$, we continue our construction to find points $\tilde{x}^{(2)} \in \Sigma_{2}, \tilde{x}^{(3)} \in \Sigma_{1}$, ... The sequence of points $\tilde{x}^{(j)}$ will be finite if $\tilde{x}^{(2 m)} \notin \Sigma_{2}$ or $\tilde{x}^{(2 m-1)} \notin \Sigma_{1}$ for some $m \geq 1$. Otherwise, the sequence $\tilde{x}^{(j)}$ will contain infinite number of terms. If it is finite, say $j=1, \ldots, N$, then we apply our previous argument to the point $\tilde{x}^{(N)}$ and to the plane $\Sigma_{i}$, where $i=1$ if $N$ is odd and $i=2$ if $N$ is even.

Assume now that the constructed sequence of points $\tilde{x}^{(j)}=\left(x_{0}^{\prime}, \tilde{y}^{(j)}\right), j=1,2, \ldots$, is infinite. By our construction we have $\left|\tilde{y}^{(j+1)}\right| \geq\left|\tilde{y}^{(j)}\right| \sec (\gamma \pi)$ for all $j=1,2, \ldots$. Therefore,

$$
\left|\tilde{x}^{(j)}\right| \rightarrow \infty \quad \text { as } j \rightarrow \infty .
$$

Since for every $j$ there is an index $m(j)$ and a point $z^{(j)} \in \Omega_{m(j)}$ such that $\left|z^{(j)}-\tilde{x}^{(j)}\right| \leq$ 1 the latter limit relation contradicts our assumption that $\Omega \subset B^{(n)}$.

This completes the proof of the theorem in the case (i).

In the case (ii) the proof is simpler. As in the case (i), we consider two subcases.

(1) First, we suppose that $B_{\varepsilon_{0}}^{(n)}\left(x_{0}\right) \cap \Omega_{m_{s}}=\emptyset$ for some sufficiently small $\varepsilon_{0}>0$ and some infinite subsequence of indices $m_{s}, s=1,2, \ldots$

Fix $\delta_{0}>0$ sufficiently small. Then let $K$ be a compact subset of $\Omega$ such that

$$
\mathcal{L}^{n}(\Omega \backslash K)<\delta_{0}
$$

Let $K^{*}=S(K)$ and let $K_{j}, j=1,2, \ldots$, be successive symmetrizations of $K$ defined by (3.7) and (3.8). Since $K_{m_{s}} \subset \Omega_{m_{s}}$, we have

$$
B_{\varepsilon_{0}}^{(n)}\left(x_{0}\right) \cap K_{m_{s}}=\emptyset .
$$

Since $x_{0} \in \partial \Omega^{*}$ there is $\delta_{1}>0$ such that

$$
\mathcal{L}^{n}\left(B_{\varepsilon_{0}}^{(n)}\left(x_{0}\right) \cap \Omega^{*}\right) \geq \delta_{1} .
$$

This together with (12.24) implies that

$$
\mathcal{L}^{n}\left(B_{\varepsilon_{0}}^{(n)}\left(x_{0}\right) \cap K^{*}\right) \geq \delta_{2}
$$

for some $\delta_{2}>0$ if $\delta_{0}>0$ in (12.24) is sufficiently small.

Applying equation (3.11) to the compact set $K$, we obtain

$$
\mathcal{L}^{n}\left(K_{m_{s}} \triangle K^{*}\right) \rightarrow 0 \text { as } s \rightarrow \infty .
$$

One can easily see that equations (12.26) and (12.27) contradict (12.25). This proves the theorem in the case under consideration.

(2) Suppose now that $\varepsilon_{0}>0$ and $x_{0}=\left(x_{0}^{\prime}, y_{0}\right)$ are such that

$$
B_{\varepsilon_{0}}^{(n)}\left(x_{0}\right) \subset \Omega_{m_{s}}
$$

for some infinite subsequence $m_{s}, s=1,2, \ldots$ This implies that, $\Omega_{m_{s}}\left(x_{0}^{\prime}\right) \neq \emptyset$ and therefore $\Omega\left(x_{0}^{\prime}\right) \neq \emptyset$ and $\Omega^{*}\left(x_{0}^{\prime}\right) \neq \emptyset$. Since $\Omega^{*}\left(x_{0}^{\prime}\right)$ is a $k$-dimensional ball there is $r>0$ such that $\Omega^{*}\left(x_{0}^{\prime}\right)=B_{r}^{(k)}$. Since $x_{0} \notin \Omega^{*}$, we have $r \leq\left|y_{0}\right|$. This implies that

$$
\mathcal{L}^{k}\left(\left(B_{\varepsilon_{0}}^{(n)}\left(x_{0}\right) \backslash \Omega^{*}\right)\left(x_{0}^{\prime}\right)\right) \geq \delta_{1}
$$


for some $\delta_{1}>0$. Here $\left(B_{\varepsilon_{0}}^{(n)}\left(x_{0}\right) \backslash \Omega^{*}\right)\left(x_{0}^{\prime}\right)$ denotes the slice of $B_{\varepsilon_{0}}^{(n)}\left(x_{0}\right) \backslash \Omega^{*}$ at $x_{0}^{\prime}$. For every arbitrary small $\delta_{2}>0$ there exists a $k$-dimensional compact set $K \subset \Omega\left(x_{0}^{\prime}\right)$ such that

$$
\mathcal{L}^{k}\left(\Omega\left(x_{0}^{\prime}\right) \backslash K\right)=\mathcal{L}^{k}\left(\Omega^{*}\left(x_{0}^{\prime}\right) \backslash K^{*}\right)<\delta_{2},
$$

where $K^{*}$ denotes the $(k, k)$-Steiner symmetrization of $K$.

Applying equation (3.11) of Theorem 3.1 to $K$, we obtain

$$
\mathcal{L}^{k}\left(K_{s} \triangle K^{*}\right) \rightarrow 0 \text { as } s \rightarrow \infty,
$$

where $K_{s}=\left(\left(\left\{x_{0}^{\prime}\right\} \times K\right)_{s}\right)\left(x_{0}^{\prime}\right)$ denotes the slice at $x_{0}^{\prime}$ of the symmetrized set $\left(\left\{x_{0}^{\prime}\right\} \times K\right)_{s}$ defined by formulas (3.7) and (3.8).

By the monotonicity property of symmetrization, we have $K_{m_{s}} \subset \Omega_{m_{s}}\left(x_{0}^{\prime}\right)$. This and (12.30) imply that

$$
\mathcal{L}^{k}\left(\Omega_{m_{s}}\left(x_{0}^{\prime}\right) \backslash K_{m_{s}}\right)<\delta_{2} .
$$

Finally, (12.28), (12.29), and (12.32) imply that there is a constant $\delta_{3}>0$ such that

$$
\mathcal{L}^{k}\left(K_{m_{s}} \backslash \Omega^{*}\left(x_{0}^{\prime}\right)\right) \geq \delta_{3}
$$

for all sufficiently large $s$. Now one can easily see that (12.33) contradicts (12.30) and (12.31) if $\delta_{2}>0$ in (12.30) is sufficiently small.

The proof of equation (3.10) of Theorem 3.1 is finished.

To prove (3.11) for $\Omega \in \mathcal{G}_{n, b}$, we fix $x^{\prime} \in \mathbb{R}^{n-k}$ such that the slice $\Omega\left(x^{\prime}\right)$ is not empty. Considering the restrictions of the symmetrizations $S, S_{1}$, and $S_{2}$ to the slice $\mathbb{R}^{n}\left(x^{\prime}\right)$, we obtain

$$
\lim _{j \rightarrow \infty} d\left(\partial \Omega_{j}\left(x^{\prime}\right), \partial \Omega^{*}\left(x^{\prime}\right)\right)=0
$$

Since $\Omega^{*}\left(x^{\prime}\right)$ is an open $k$ dimensional ball and $\mathcal{L}^{k}\left(\Omega^{*}\left(x^{\prime}\right)\right)=\mathcal{L}^{k}\left(\Omega_{j}\left(x^{\prime}\right)\right)$, equation (12.34) implies (3.11) in the case of bounded open sets.

The proof of Theorem 3.1 is now complete.

Remark 12.1. One can easily show that (3.11) remains valid even for unbounded sets $\Omega$ if the measure of the corresponding slice is finite, i.e. if $\mathcal{L}^{k}\left(\Omega\left(x^{\prime}\right)\right)<\infty$.

In contrast, simple examples of unbounded domains $\Omega$ with a finite measure, $\mathcal{L}^{n}(\Omega)<$ $\infty$, show that (3.10) is not true for unbounded open sets in general.

\section{REFERENCES}

[1] S. Abramovich, Monotonicity of eigenvalues under symmetrization, SIAM J. Appl. Math. 28 (1975), 350-361.

[2] L. V. Ahlfors, Conformal Invariants: Topics in Geometric Function Theory, McGraw-Hill: New York, 1973.

[3] H. W. Alt, Lineare Funktionalanalysis, second edition, Springer-Verlag 1992.

[4] A. Alvino, P.-L. Lions, G. Trombetti, Comparison results for elliptic and parabolic equations via symmetrization: A new approach, Differential and Integral Equations 4 (1991), 25-50. MR 91h:35023

[5] A. Alvino, P.-L. Lions, G. Trombetti, Comparison results for elliptic and parabolic equations via Schwarz symmetrization, Ann. Inst. Poincaré, Anal. non lineáire 7(2) (1990), 37-65. MR 91f:35022 
[6] A. Baernstein II, A unified approach to symmetrization, in: Partial differential equations of elliptic type (eds) A. Alvino et al. (1995) Symposia matematica (Cambridge Univ. Press) vol. 35 , pp. $47-91$.

[7] A. Baernstein II and B. A. Taylor, Spherical rearrangements, subharmonic functions, and *functions in n-space, Duke Math. J. 43 (1976), 245-268.

[8] W. Beckner, Sobolev inequalities, the Poisson semigroup and analysis on the sphere $S^{n}$, Proc. Nat. Acad. Sci. U.S.A. 89 (1992), 4816-4819. MR 93d:26018

[9] D. Betsakos, Polarization, conformal invariants, and Brownian motion, Ann. Acad. Sci. Fenn. Math. 23 (1998), no. 1 59-82.

[10] D. Betsakos, Polarization, ccontinuous Markov processes, and second order elliptic equations, Indiana Univ. Math. J. 53 (2004), no. 2, 331-345.

[11] W. Blaschke, Kreis und Kugel, Chelsea Publishing Company, New York, 1949.

[12] F. Brock, Continuous Steiner-symmetrization, Math. Nachr. 172 (1995), 25-48.

[13] F. Brock, Continuous rearrangement and symmetry of solutions of elliptic problems, Proc. Indian Acad. Sci. (Math. Sci.) 110 (2) (2000), 157-204.

[14] F. Brock and A. Yu. Solynin, An approach to symmetrization via polarization, Trans. Amer. Math. Soc. 352 (4) (2000), 1759-1796.

[15] V. N. Dubinin, Transformation of functions and Dirichlet's principle, Math. Zametki 38 (1985), 49-55; English transl. in Math. Notes 38 (1985), 539-342.

[16] V. N. Dubinin, Capacities and geometric transformations in n-space, Geom. and Funct. Anal. 3 (1993), 342-369.

[17] V. N. Dubinin, Symmetrization in geometric theory of functions of complex variables, Uspehi Mat. Nauk 49 (1994), 3-76; English transl. in

[18] B. Gidas, W. M. Ni \& L. Nirenberg, Symmetry and related properties via the maximum principle, Comm. Math. Phys., 68 (1979), 209-243.

[19] J. Kačur, Method of Rothe in Evolution Equations, Teubbner-Verlag, Leipzig 1985. MR 87j:35004

[20] B. Kawohl, Rearrangements and convexity of level sets in PDE, Springer Lecture Notes 1150 (1985).

[21] B. E. Levitskii, k-symmetrization and extremal rings, (in Russian) Math. Anal. Kubanskii State Univ. Krasnodar, 1971, 35-40.

[22] A. McNabb, Partial Steiner symmetrization and some conduction problems, J. Math. Anal. Appl. 17 (1967), 221-227.

[23] M. Marcus, Radial averiging of domains, estimates for Dirichlet integrals and applications, J. d' Analyse 27 (1974), 47-93.

[24] G. Pólya and G. Szegö, Isoperimetric Inequalities in Mathematical Physics, Ann. Math. Studies 27, Princeton Univ. Press, 1952.

[25] J. Sarvas, Symmetrization of condensers in n-space, Ann. Acad. Sci. Fenn. Ser. A1 522 (1972), $1-44$.

[26] J. Serrin, A symmetry problem in potential theory, Arch. Rational Mech. Anal. 43 (1971), 304318.

[27] A. Yu. Solynin, Continuous symmetrization of sets, Zap. Nauchn. Seminarov LOMI Akad. Nauk SSSR 185 (1990), 125-139; English translation in: J. Soviet Math. 59 no. 6 (1992), 1214-1221.

[28] A. Yu. Solynin, Functional inequalities via polarization, Algebra i Analiz 6 (1996), 148-185; English transl. in St. Petersburg Math. J. 8 (1997), 1015-1038.

[29] A. Yu. Solynin, Ordering of sets, hyperbolic metric, and harmonic measure, Zap Nauchn. Sem. POMI 237 (1997), 129-147; English transl. in J. Math. Sci. (New York) 95 (1999), no. 3, 22562266. MR 2000d:30068

[30] J. Steiner, Gesammelte Werke, Vol.2, Reimer-Verlag, Berlin, 1882.

[31] G. Szegö, , Arch. Rational Mech. Anal. 43 (1971), 304-318.

[32] G. Talenti, The standard isoperimetric theorem, in: Handbook of Convex Geometry, Volume A, North-Holland, Amsterdam 1993, 73-124. MR 94h:49065

[33] V. Wolontis, Properties of conformal invariants, Amer. Journ. Math. 74 (1952), 587-606.

Department of Mathematics and Statistics, Texas Tech University, Box 41042, Lubbock, TX 79409, USA

E-mail address: alex.solynin@ttu.edu 